\title{
A DIFFRACTION BASED STUDY OF THE DEFORMATION MECHANISMS IN ANOMALOUSLY DUCTILE B2 INTERMETALLICS
}

\author{
A Dissertation \\ Presented to \\ The faculty of the School of Engineering and Applied Science \\ University of Virginia
}

\begin{abstract}
In Partial Fulfillment
of the requirements for the Degree

Doctor of Philosophy (Materials Science and Engineering)

by
\end{abstract}

Rupalee Prashant Mulay

December 2011 


\section{APPROVAL SHEET}

This dissertation is submitted in partial fulfillment of the

requirements for the degree of

Doctor of Philosophy (Materials Science and Engineering)

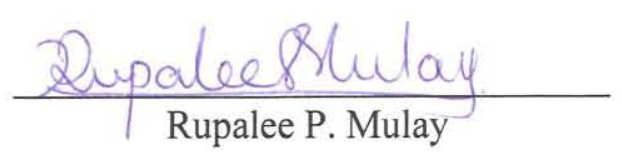

This dissertation has been read and approved by the examining Committee:

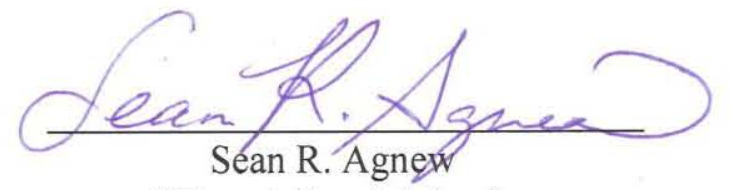

(Dissertation Advisor)

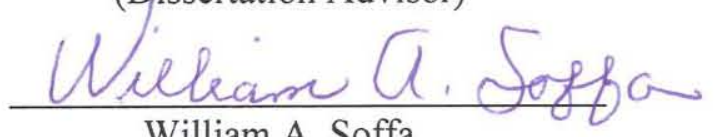

William A. Soffa

(Committee Chairperson)
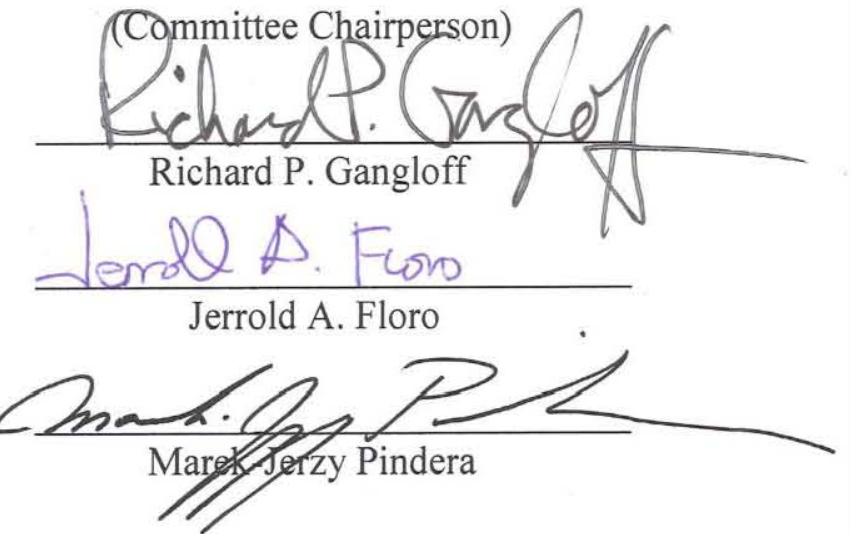

Accepted for the School of Engineering and Applied Science:

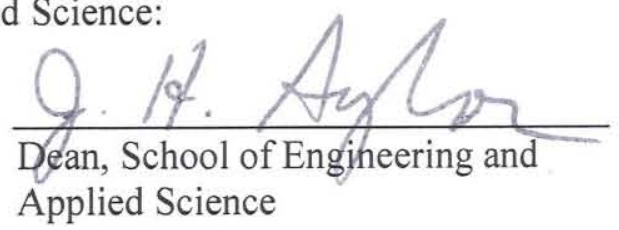

December, 2011 


\begin{abstract}
For many decades, the brittle nature of most intermetallic compounds (e.g. NiAl) has been the limiting factor in their practical application. Many B2 $(\mathrm{CsCl}$ prototypical structure) intermetallics are known to exhibit slip on the $<001>\{110\}$ slip mode, which provides only 3 independent slip systems and, hence, is unable to satisfy the von Mises (a.k.a. Taylor ) criterion for polycrystalline ductility. As a result, inherent polycrystalline ductility is unexpected. Recent discovery of a number of ductile B2 intermetallics has raised questions about possible violation of the von Mises criterion by these alloys. These ductile intermetallic compounds are MR (metal (M) combined with a rare earth metal or group IV refractory metal (R)) alloys and are stoichiometric, ordered compounds. Single crystal slip trace analyses have only identified the presence of $<100>\{011\}$ or $<100>\{010\}$ slip systems. More than 100 other B2 MR compounds are known to exist and many of them have already been shown to be ductile (e.g., CuY, AgY, CuDy, CoZr, CoTi, etc.). Furthermore, these alloys exhibit a large Bauschinger effect.

The present work uses several diffraction based techniques including electron back scattered diffraction (EBSD), X-ray diffraction (XRD) and in-situ neutron diffraction; in conjunction with scanning electron microscopy (SEM), transmission electron microscopy (TEM), mechanical testing, and crystal plasticity modeling, to elucidate the reason for ductility in select B2 alloys, explore the spread of this ductility over the B2 family, and understand the Bauschinger effect in these alloys. Several possible explanations (e.g., slip of <111> dislocations, strong texture, phase transformations and twinning) for the anomalous ductility were explored. An X-ray
\end{abstract}


diffraction based analysis ruled out texture, phase purity and departure from order as explanations for the anomalous ductility in MR alloys. In-situ neutron diffraction and post deformation SEM, EBSD, and TEM were unable to detect any evidence for phase transformations in CoTi and CoZr. Also, post deformation characterization did not reveal any evidence of twinning.

However, TEM based $\mathbf{g} \cdot \mathbf{b}$ analysis and EBSD based in-grain misorientation axis (IGMA) analysis showed that beyond a transition in the strain hardening behavior in CoTi, slip modes involving dislocations with $\langle 110\rangle$ and $\langle 111\rangle$ Burgers vectors are activated. The slip of such dislocations can reduce stress concentrations that could otherwise lead to premature fracture, thus providing a satisfying explanation for the anomalous ductility of CoTi and related compounds, like CoZr. Dislocation self-energy calculations accounting for elastic anisotropy suggest that the choice of slip direction in these alloys is mobility-, rather than source-, limited.

The reach of this "ductilizing effect" over B2 alloys was explored by producing, characterizing, and testing a number of simple metal-rare earth metal compounds, namely $\mathrm{MgY}, \mathrm{MgNd}$ and $\mathrm{MgCe} . \mathrm{MgR}$ intermetallics with the B2 structure were found to be brittle and exhibit a cleavage type fracture indicating that the ductilizing effect is not as widespread as was initially thought. $\mathrm{MgY}$ and $\mathrm{MgNd}$ were found to primarily cleave along the $\{100\}$ planes, while MgCe was found to cleave on the $\{111\}$ planes.

A large Bauschinger effect was observed in several of the anomalously ductile B2 compounds, such that the material actually begins to yield in the reverse direction on unloading. When only the primary slip mode $\langle 100>\{011\}$ is active in CoZr (prior to a transition in strain hardening), the buildup of intergranular stresses is large and is chiefly 
responsible for the observed Bauschinger effect. However, past the aforementioned transition in strain hardening, the effect of intergranular stresses diminishes. The results demonstrate that the activation of hard, secondary slip modes causes the internal strains to develop more uniformly among the grains, thus reducing the intergranular stresses which cause the Bauschinger effect. Crystal plasticity modeling, which accounts for the initial paucity of independent slip modes and allows for the activation of complementary hard slip modes, reproduces these trends in the Bauschinger effect and provides additional evidence that the experimental observations have correctly identified the cause of the anomalous ductility. 


\section{Collaborative Contribution}

Each chapter from 2-5 constitutes an individual journal paper. Chapters 2 and 5 contain authors other than the dissertation author R.P. Mulay and advisor S.R. Agnew. Chapter 2 has four additional authors. J.A. Wollmershauser helped with the polycrystal modeling, M.A. Heisel was an undergraduate student in the group who carried out some of the X-ray diffraction scans, H. Bei provided figure 2.5 and A.M. Russell provided some of the alloys used. Chapter 5 has one additional author, B. Clausen, who was the instrument scientist at Los Alamos National Laboratory (LANL), where the in-situ neutron diffraction experiments were performed. 


\section{Acknowledgements}

I would like to express my sincere thanks to my advisor Prof. Sean Agnew for his guidance and support over the past four years. His zest for science and teaching is infectious and he has always helped me put forth my best effort. I would also like to thank Prof. William Soffa for many helpful discussions.

I am grateful to my colleagues Drs. James Wollmershauser, Vipul Gupta and YunJo Ro for many constructive discussions and assistance with using equipment. I am also thankful to John Polesak, Dr. Jason Hadorn, Chris Amaro and Dr. Babak Raeisinia. My gratitude also goes out to my friends and colleagues in the department for a great working atmosphere.

I would also like to thank my committee members, Professors Sean Agnew, William Soffa, Richard Gangloff, Jerry Floro and Marek-Jerzy Pindera for their constructive comments and help.

I am grateful to Richard White (nano-scale materials characterization facility) for training me to use various microscopes and equipment and NSF CAREER Grant DMR0547981 for funding this research.

Finally, a big thank you to my family and friends, particularly my parents and my brother. I can always count on them for their support and guidance. 


\section{Table of Contents}

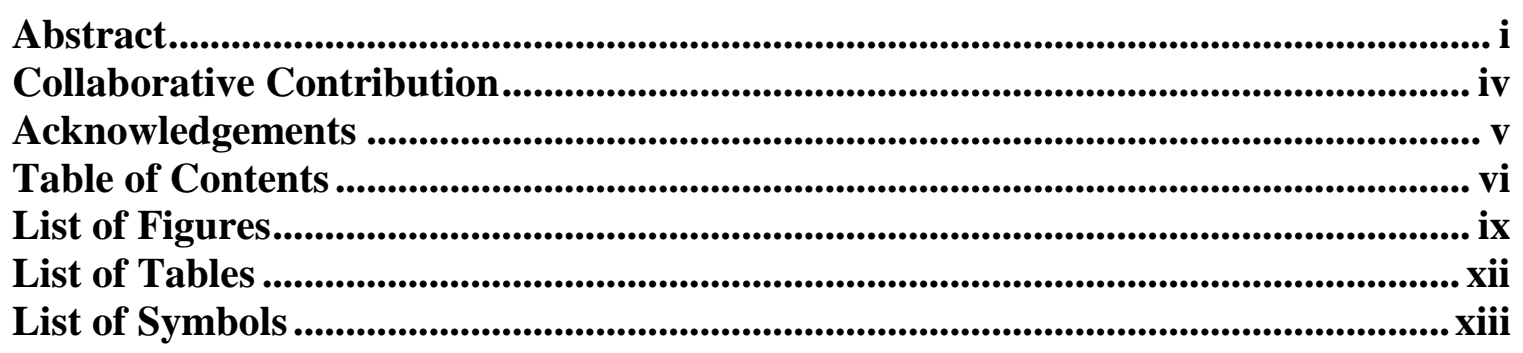

1.Introduction ............................................................................................................................ 1

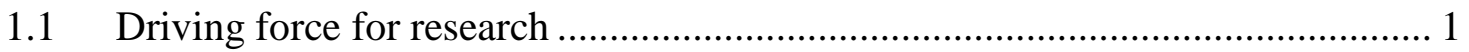

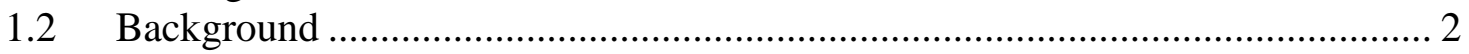

1.2.1 Archetypal behavior in the B2 family at room temperature ..................... 2

1.2.2 Anomalously ductile B2 intermetallics.................................................... 3

1.2.3 The Bauschinger effect ……………………....................................... 8

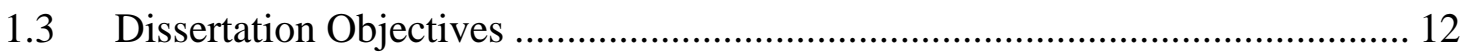

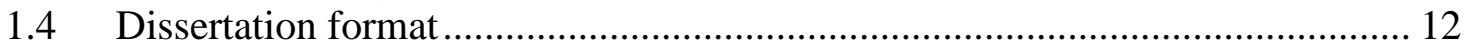

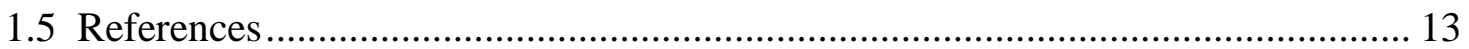

2. X-ray diffraction study of the phase purity, order, and texture of ductile B2 intermetallics ............................................................................................................................ 18

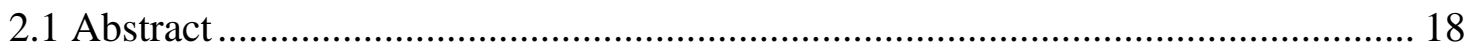

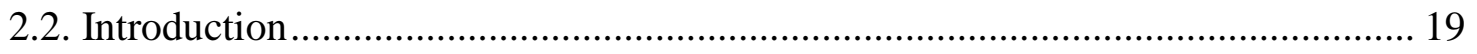

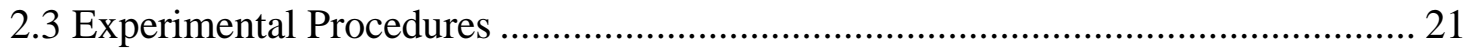

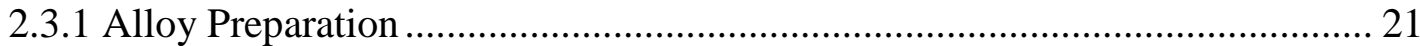

2.3.2 Quantitative Phase Analysis ....................................................................... 24

2.3.3 Order Parameter Determination ................................................................ 25

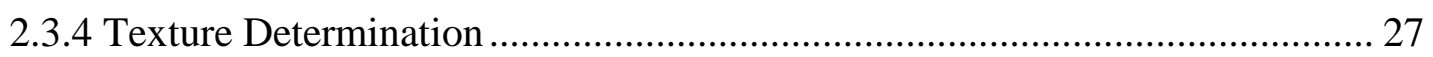

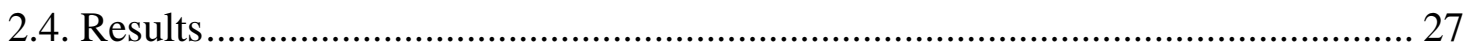

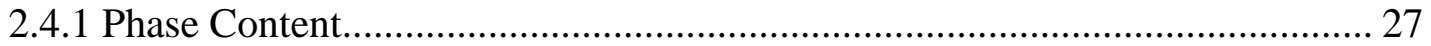

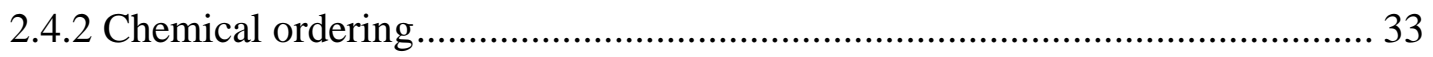

2.4.3 Crystallographic Texture ……………………………................................ 37

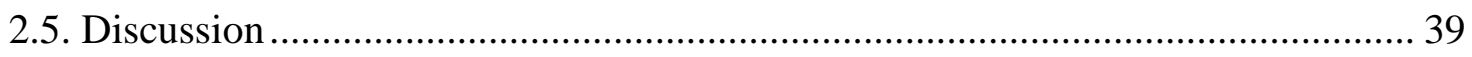

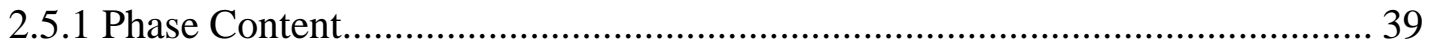

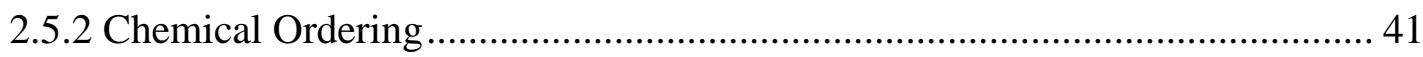

2.5.3 Crystallographic Texture ……………………........................................ 42

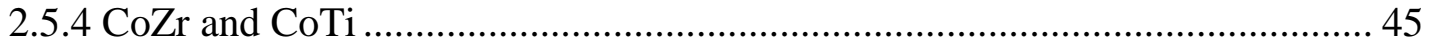




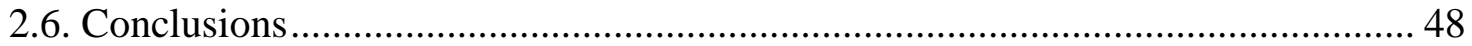

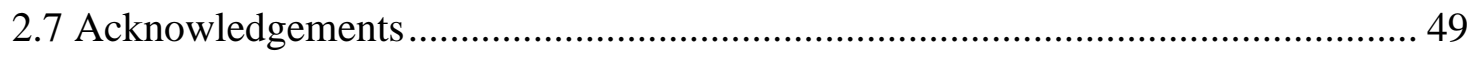

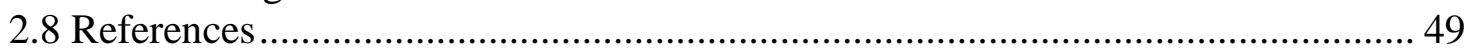

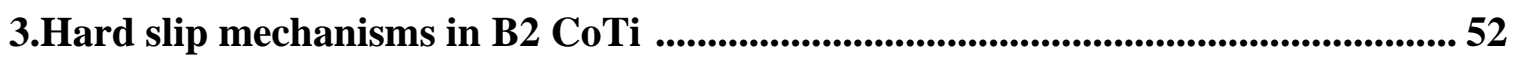

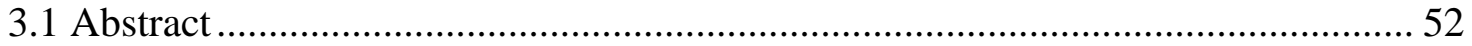

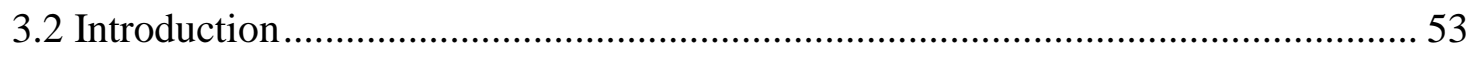

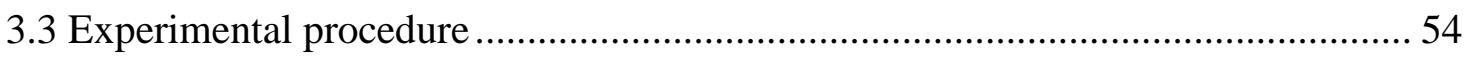

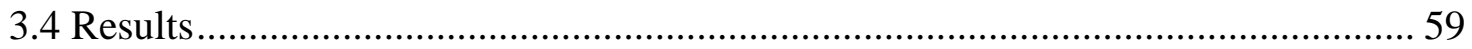

3.4.1 Optical metallography and mechanical tests..............................................5

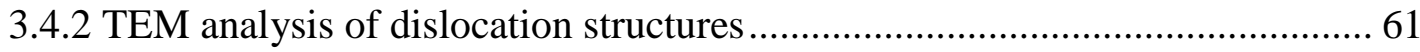

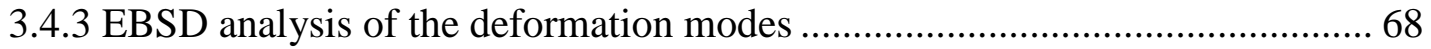

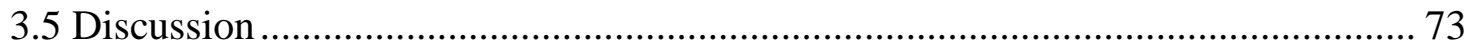

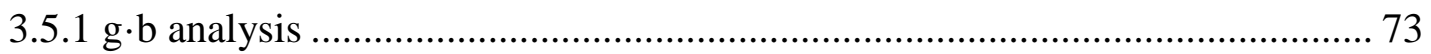

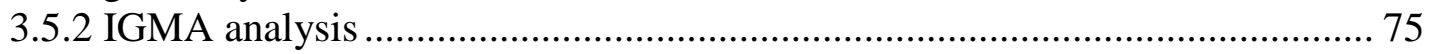

3.5.3 Source and mobility of dislocations...................................................... 76

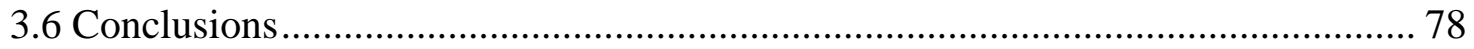

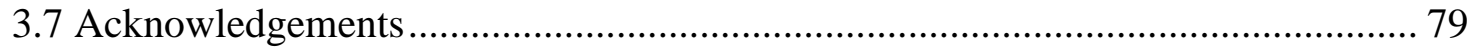

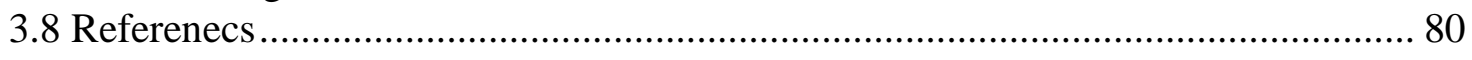

4. The fracture behavior of B2 structured MgR intermetallics..................................... 83

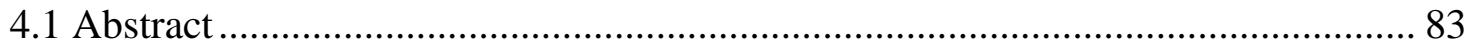

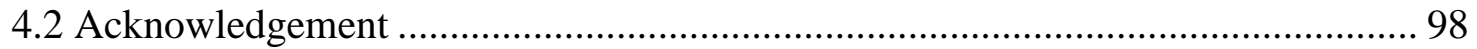

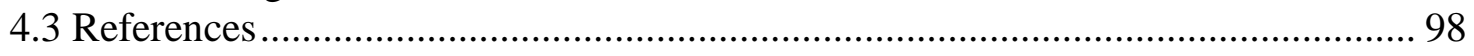

5. In situ neutron diffraction study of the Bauschinger effect in B2 structured CoZr.............................................................................................................. 101

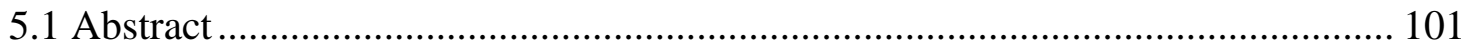

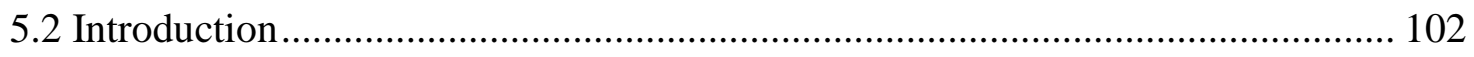

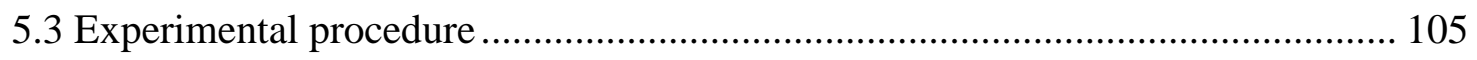

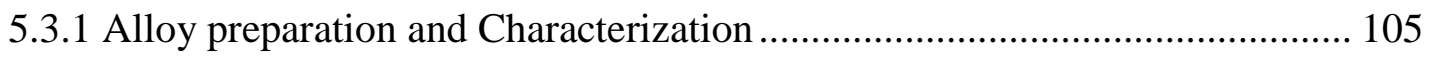

5.3.2 Compression tests and in-situ neutron diffraction ..................................... 106

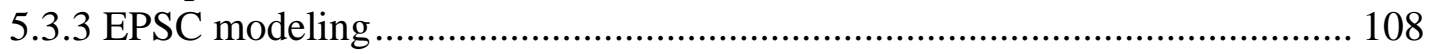

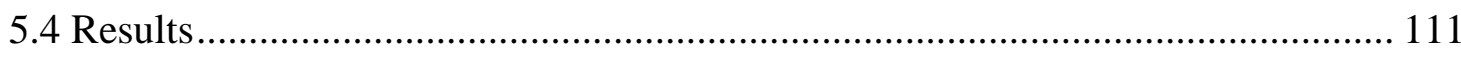

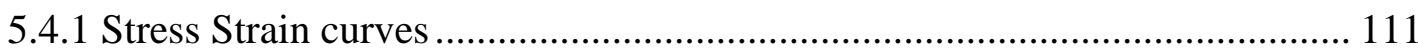

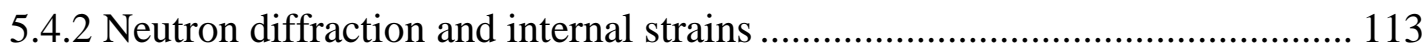

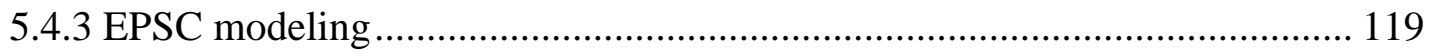

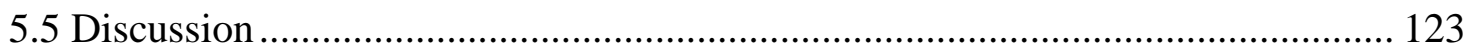




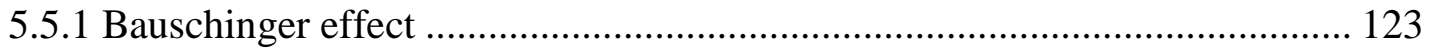

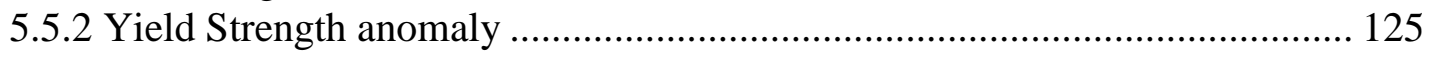

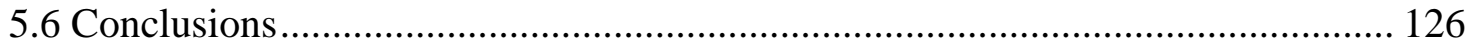

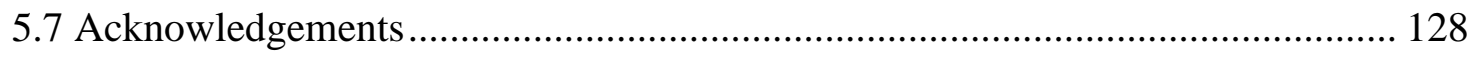

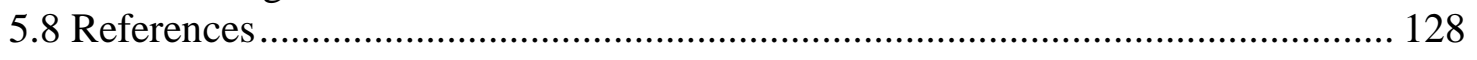

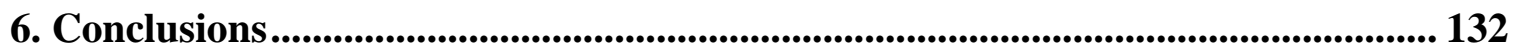

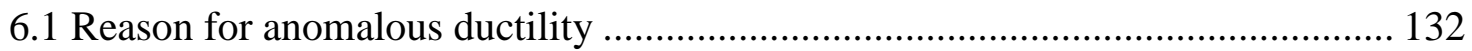

6.2 Breadth of ductility ................................................................................. 134

6.3 Bauschinger effect in anomalously ductile B2 intermetallics.......................... 135

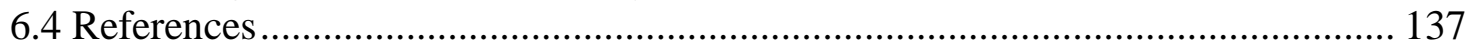

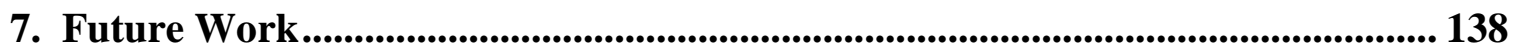

7.1 A brief discussion of the implications of the present conclusions on the understanding of yield anomaly in B2 compounds......................................... 138

7.2 Dislocation core structures and mobilities ................................................. 140

7.3 Incorporation of intragranular stresses in the EPSC model ........................... 141

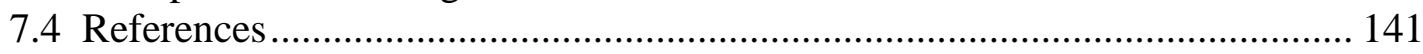




\section{List of Figures}

Figure 1.1: The $\mathrm{B} 2$ ( $\mathrm{CsCl}$ prototype) crystal structure showing the possible Burgers vectors of perfect dislocations.

Figure 1.2: Phase diagram of the Ag-Y system showing the B2 AgY compound at 50-50 at\% composition [19]. Reprinted with permission of ASM intermational. 3 Figure 1.3: Stress- strain curves of some of the ductile MR compounds. Reprinted by permission from Macmillan Publishers Ltd: [Nature Materials] (9), copyright (2003) .... 4 Figure 1.4: A diagram showing the existing B2 MR compounds. The present work examines the ductility in MgR intermetallics. Reprinted by permission from Macmillan Publishers Ltd: [Nature Materials] (9), copyright (2003).

Figure 1.5: Stress- Strain curves for CoZr and CoTi showing the transition in strain hardening. With kind permission from Springer Science+Business Media [39]............... 7

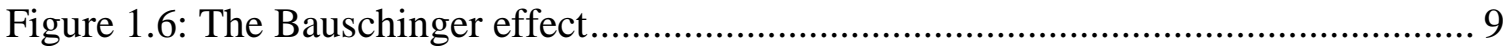

Figure 1.7: Intergranular stresses can cause the Bauschinger effect ............................. 10

Figure 1.8: Intragranular stresses can be responsible for the Bauschinger effect............. 11

Figure 2.1: X-ray Diffraction patterns used in the phase analysis of a)AgY b)AgEr c)CuY

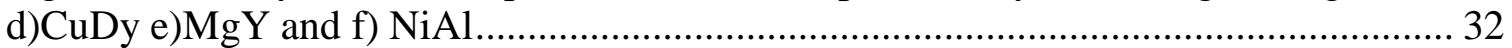
Figure 2.2: X-ray Diffraction patterns used in the order parameter determination of

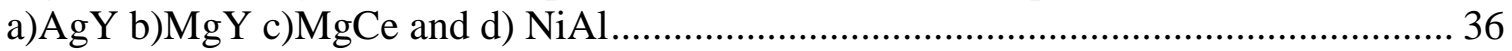
Figure 2.3: A typical plot of MgY used to obtain the temperature factor. ...................... 36 Figure 2.4: Pole figures representing the texture of rolled a) $\mathrm{AgY} \mathrm{b}$ ) $\mathrm{AgEr}$ and c) $\mathrm{CuY}$, with rolling direction vertical and transverse direction horizontal, and d) cast $\mathrm{MgY}$,

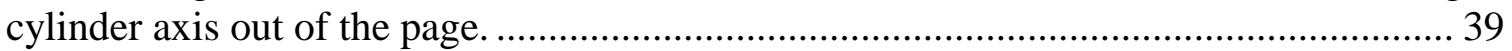
Figure 2.5: a) Micrograph of $\mathrm{AgY}$ showing second phases $\mathrm{Ag}_{2} \mathrm{Y}$ and $\mathrm{Y}_{2} \mathrm{O}_{3}$. b) Micrograph of AgY showing interaction between cracks and second phase particles.... 40 Figure 2.6: EPSC modeling of the mechanical behavior of AgY, a) Stress vs. Strain curve b) Internal strains, which show that the stresses built up are lower in the presence of a moderate texture.

Figure 2.7: Pole figures of a) $\mathrm{CoZr}$ and b) CoTi with the extrusion axis out of the page. Both alloys have a weak texture with $<111>$ parallel to the extrusion axis. .................... 46 Figure 2.8: X-ray diffraction patterns of a) CoZr and b) CoTi.................................... 47

Figure 3.1: Compressive and tensile stress-strain curves of CoTi [7] .......................... 54

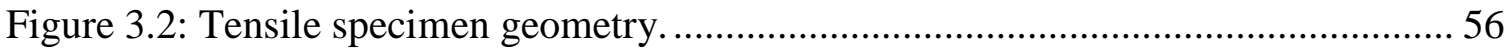
Figure 3.3: Optical micrograph showing the microstructure in recrystallized CoTi. The

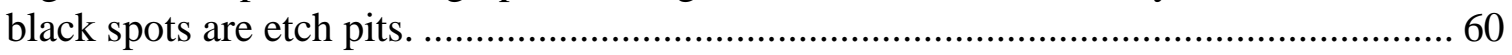
Figure 3.4: Bright field images of CoTi at different strain levels, a) recrystallized and b) 9

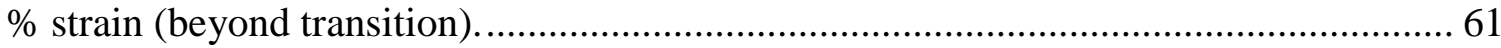


Figure 3.5: Series of two-beam bright field images of dislocations in CoTi deformed to 7 $\%$ strain. The electron beam direction is near [001] for images a, b, c and near [111] for

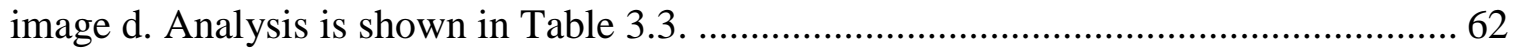
Figure 3.6: Series of two-beam bright field images of dislocations in CoTi deformed to 9 $\%$ strain. The electron beam direction is near [111] for images a, b, c and near [001] for images $\mathrm{d}$ and e. Analysis is shown in Table 3.4. 63 Figure 3.7: Series of two-beam bright field images of dislocations in CoTi deformed to 9 $\%$ strain. The electron beam direction is near [001] for images a, b, e and near [111] for images $\mathrm{c}$ and $\mathrm{d}$. Analysis is shown in Table 3.5. 65 Figure 3.8: Series of two-beam bright field images of dislocations in CoTi deformed to 9 $\%$ strain. The electron beam direction is near [001] for images a, b, d and near [111] for

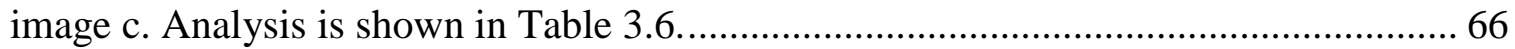
Figure 3.9: Preferred slip mode in B2 alloys based on schmid factor. All slip modes are assumed to have the same critical resolved shear stress . 67 Figure 3.10: OIM of CoTi tensile sample which failed prior to the transition in strain hardening. 68

Figure 3.11: Irreducible stereographic triangle showing, a) the in-grain misorientation axis for grains A and B (Fig.3.10) respectively. The maximum and minimum intensities for each plot are indicated. b) The orientation of the loading axis in grains A and B..... 69 Figure 3.12: OIM of CoTi compression sample deformed beyond the transition in strain hardening 71

Figure 3.13: Irreducible stereographic triangle showing, a) the in-grain misorientation axis for grains $\mathrm{C}$ and D (Fig.3.12) respectively. The maximum and minimum intensities for each plot are indicated. b) The orientation of the loading axis in grains $\mathrm{C}$ and D..... 72 Figure 3.14: Series of two-beam bright field images of dislocations in CoZr deformed to $20 \%$ strain. The electron beam direction is near [110] for image a and near [111] for image b, c and d. g.b analysis shows the presence of a [111] dislocation. 75

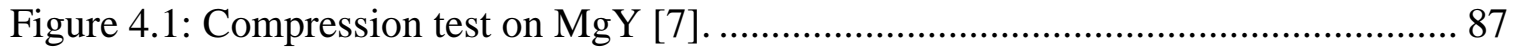
Figure 4.2: Fracture surface of a) $\mathrm{NiAl}$ [8], b) $\mathrm{MgY}$, c) $\mathrm{MgCe}$, d) $\mathrm{CuZn}$ and e)AgY, f)

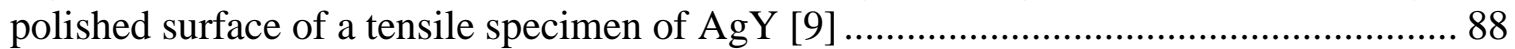
Figure 4.3: EBSD and stereology setup for a) Method 1 (polished surface EBSD), and b) Method 2 (on fracture surface EBSD).

Figure 4.4: SEM micrographs of one of the MgY facets with a sample tilt of a) $60^{\circ}$ and b) $70^{\circ}$ 91

Figure 4.5: Orientation image map superimposed on the polished surface. The fracture surface is faceted with river-like patterns typical of cleavage failure. The analysis described in the text was carried out on the facets adjacent to the polished surface. ....... 92 Figure 4.6: Irreducible triangular projection of a) $\mathrm{MgY}$ (method1) [7], b) MgNd (method

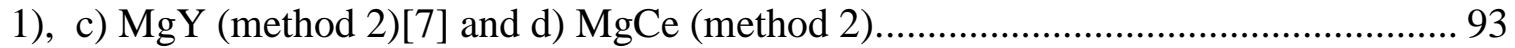
Figure 4.7: Fracture surfaces of $\operatorname{CoZr}(a, b)$ and CoTi $(c, d)$ showing intergranular fracture and cleavage, respectively, for both materials. 
Figure 5.1: Compressive stress-strain curve of CoZr at room temperature [16]. The test has been stopped intermittently and held at a constant load to collect neutron diffraction patterns, giving rise to the peaks apparent at higher stresses. Note the transition in plastic deformation at $250 \mathrm{MPa}$. 104 Figure 5.2: In-situ stress strain curves, including unload-reload cycles, at room temperature and $150^{\circ} \mathrm{C}$. 112

Figure 5.3: A plot of the "loop size" parameter as a function of stress for both the experimental and modeled stress-strain curves at room temperature and $150^{\circ} \mathrm{C}$. 113 Figure 5.4: Diffraction patterns of CoZr obtained in the "stress-free" state parallel to the loading direction at, a) room temperature and b) $150^{\circ} \mathrm{C}$. 114 Figure 5.5: Experimentally measured and simulated internal elastic strains (parallel to the loading direction) for the (111) and (210) sets of CoZr grains at room temperature for the five different loads and unloads, respectively. 116 Figure 5.6: Experimentally measured and simulated internal elastic strains (parallel to the loading direction) for the (111) and (210) sets of CoZr grains at $150{ }^{\circ} \mathrm{C}$ for the five different loads and unloads, respectively 118 Figure 5.7: a) EPSC fits superimposed on the experimental stress strain curves for value of $\beta_{\text {eff }}=1$ at room temperature. b) The part of the stress strain curve prior to the transition point has been magnified for greater clarity. 121 Figure 5.8: EPSC fits superimposed on the experimental stress strain curves for a value of $\beta_{\text {eff }}=0.82:$ a) at room temperature, b) $150{ }^{\circ} \mathrm{C}$. Part of the stress strain curve prior to the transition point has been magnified for clarity c) at room temperature and d) at 150 ${ }^{\circ} \mathrm{C}$ 


\section{List of Tables}

Table 2.1: Impurities analyzed in $\mathrm{AgY}, \mathrm{MgY}, \mathrm{MgCe}$ and $\mathrm{NiAl}$ in atomic percent $\times 10^{2} . .24$

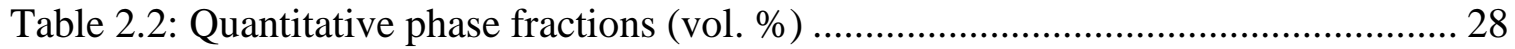

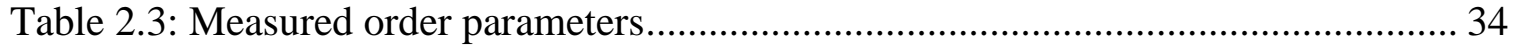

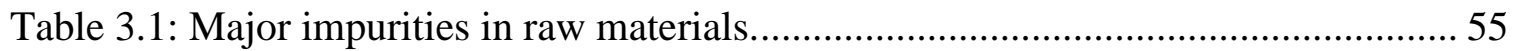

Table 3.2: In grain misorientation axis (IGMA) corresponding to the different slip

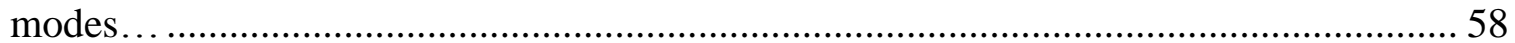

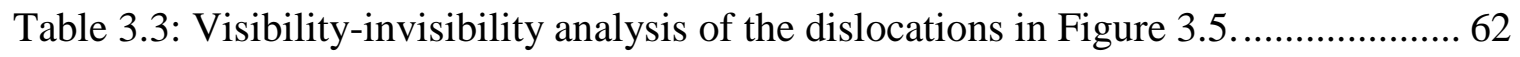

Table 3.4: Visibility-invisibility analysis of the dislocations in Figure 3.6 .................... 63

Table 3.5: Visibility-invisibility analysis of the dislocations in Figure 3.7 .....................6 65

Table 3.6: Visibility-invisibility analysis of the dislocations in Figure 3.8 …................. 66

Table 3.7: Percentage of grains showing a particular IGMA at two different strain

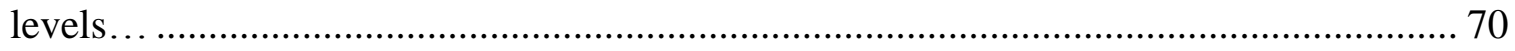

Table 4.1: Impurities analyzed in $\mathrm{MgY}, \mathrm{MgCe}$ and $\mathrm{MgNd}$ given in parts per million

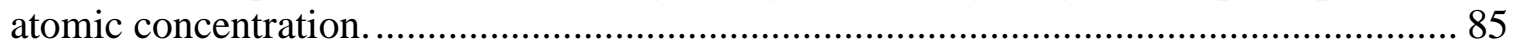

Table 5.1: Major impurities in raw materials as provided by supplier. ........................ 106 Table 5.2: Single-crystal elastic constants [32] and anisotropy factor used as inputs for EPSC modeling of CoZr both at room temperature and $150^{\circ} \mathrm{C}$................................. 120

Table 5.3: Predicted Voce hardening Parameters for the active slip modes in CoZr at room temperature

Table 5.4: Predicted Voce hardening Parameters for the active slip modes in CoZr at $150^{\circ} \mathrm{C}$ 


\section{List of Symbols}

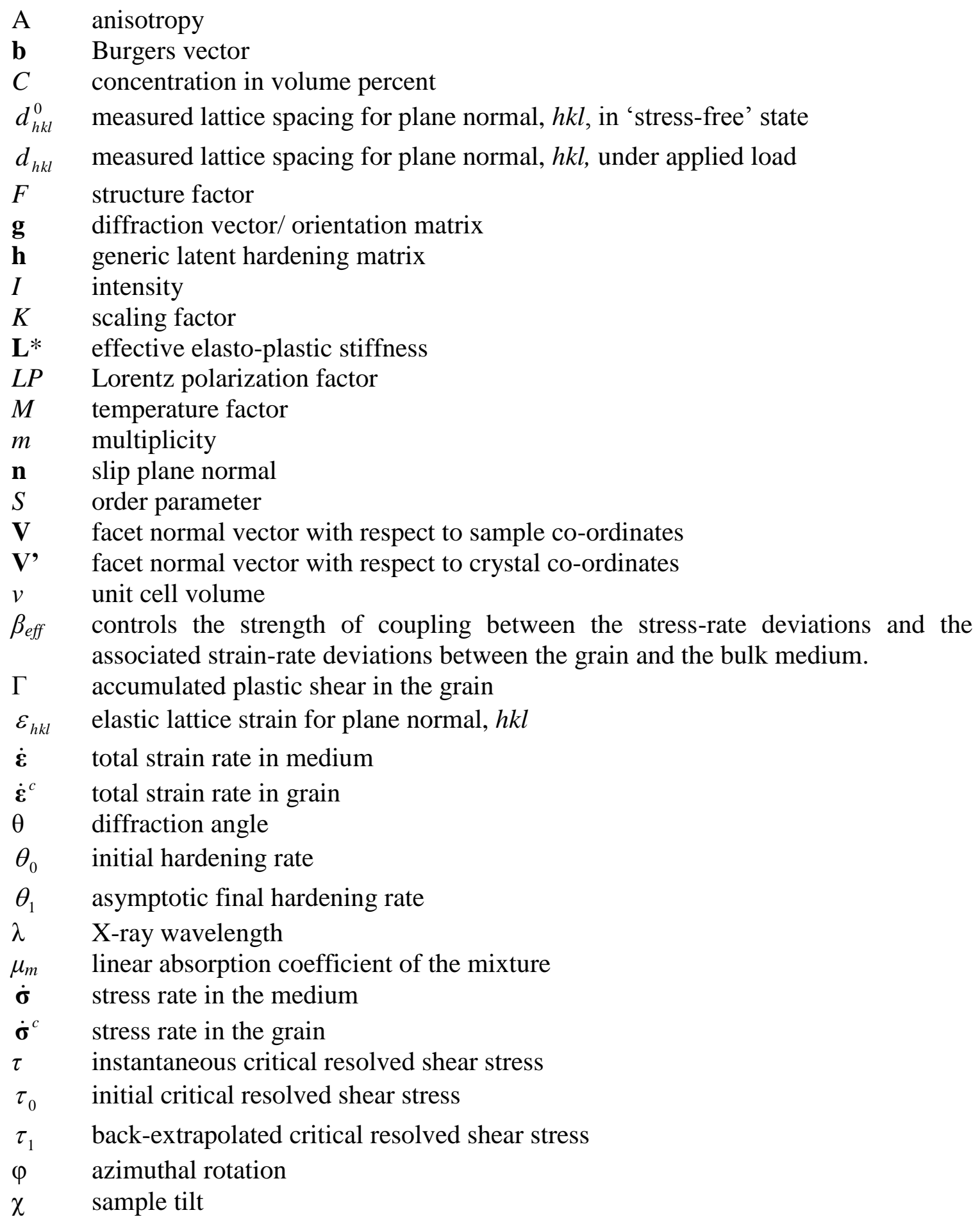




\section{Introduction}

\subsection{Driving force for research}

There has been an enormous interest in intermetallic compounds due to their attractive properties like high oxidation resistance, high melting point, etc. They have been considered as potential replacements in high temperature structural applications like turbine blades. However, their brittle nature has always been the main limitation in their practical application as structural materials [1,2]. Many B2 intermetallic compounds are known to exhibit slip on the $\langle 001>\{110\}$ slip systems at room temperature [3-6] which provides only 3 independent slip systems, and hence, is unable to satisfy the von Mises [7] (a.k.a. Taylor) criterion, which requires 5 independent slip systems for polycrystalline ductility. As a result, intrinsic polycrystalline ductility in these alloys is unexpected. It is to be noted that the von Mises criterion is a necessary but not sufficient condition for polycrystalline ductility. In addition to the availability of a sufficient number of independent slip systems, the activation of multiple slip systems must be possible, and this is referred to as slip flexibility [8]. Despite the apparent violation of von Mises criterion, select binary stoichiometric B2 intermetallics like $\mathrm{AgY}$ and $\mathrm{CoZr}$, exhibit more than $10 \%$ ductility in tension $[9,10]$. The goal of the present research is to understand this anomalous ductility in select B2 alloys with respect to the mechanisms responsible, possible reach over the B2 family, and the large Bauschinger effect exhibited by these alloys. If the mechanisms responsible for ductility in select B2 alloys are elucidated, it may be possible to reproduce them in other B2 intermetallics which are of technological interest, like NiAl. 


\subsection{Background}

Intermetallics, with the $\mathrm{B} 2(\mathrm{CsCl})$ crystal structure, form one of the largest groups of intermetallic compounds. The three possible Burgers vectors of perfect dislocations operating in B2 compounds are $\langle 100\rangle,\langle 110\rangle$ and $\langle 111\rangle$ [11] (Fig.1.1).

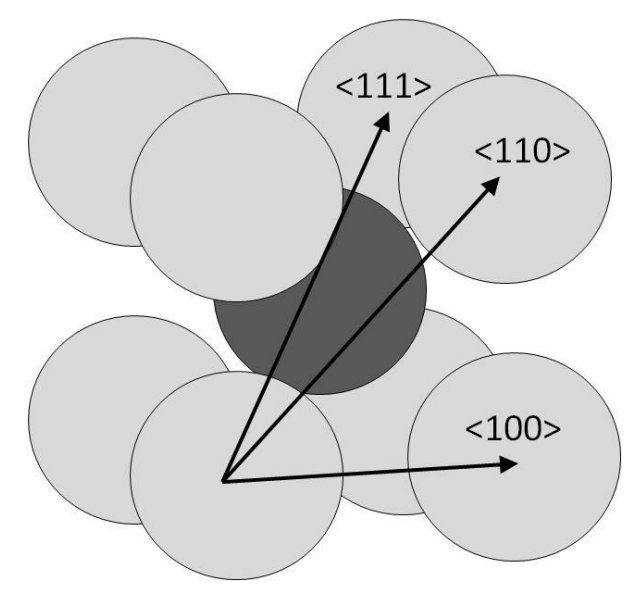

Figure 1.1: The $\mathrm{B2}$ (CsCl prototype) crystal structure showing the possible Burgers vectors of perfect dislocations.

\subsubsection{Archetypal behavior in the $\mathrm{B} 2$ family at room temperature}

$\mathrm{NiAl}$, a prototypical brittle B2 intermetallic, is known to slip on the $<001>\{110\}$ slip systems which provides only three independent slip systems $[3,4,12,13]$. The activation of a limited number of slip systems is considered to be the primary reason for the brittle nature of NiAl. NiAl has a high a/2<111> APB energy $\left(900 \mathrm{~mJ} / \mathrm{m}^{2}\right.$ [14], 500$750 \mathrm{~mJ} / \mathrm{m}^{2}$ [15]). In B2 alloys with a high a/2<111> APB energy, <111> dislocations are undissociated high energy configurations which are likely to be immobile, and hence, slip systems containing these dislocations are not found to be active. On the other hand, B2 intermetallics like CuZn which have a low APB energy (60 mJ/m² [16]), have been 
shown to deform by the slip of dislocations with <111> direction Burgers vectors $[17$, 18]. This permits them to satisfy the von Mises criterion and exhibit significant ductility.

\subsubsection{Anomalously ductile B2 intermetallics}

Recently, a significant amount of ductility has been reported in several rare-earth containing B2 intermetallic compounds by researchers at Iowa State University/Ames Laboratory [9]. These ductile intermetallics are binary line compounds (Fig.1.2) composed of a rare earth metal (R) and a simple or transition metal (M), stoichiometric and fully ordered. The most ductile MR compound observed till date is AgY (Fig.1.3), which has been reported to exhibit a tensile ductility of more than $20 \%$ at room temperature. $\mathrm{CuY}$ and $\mathrm{CuDy}$ have also shown significant ductility [9].

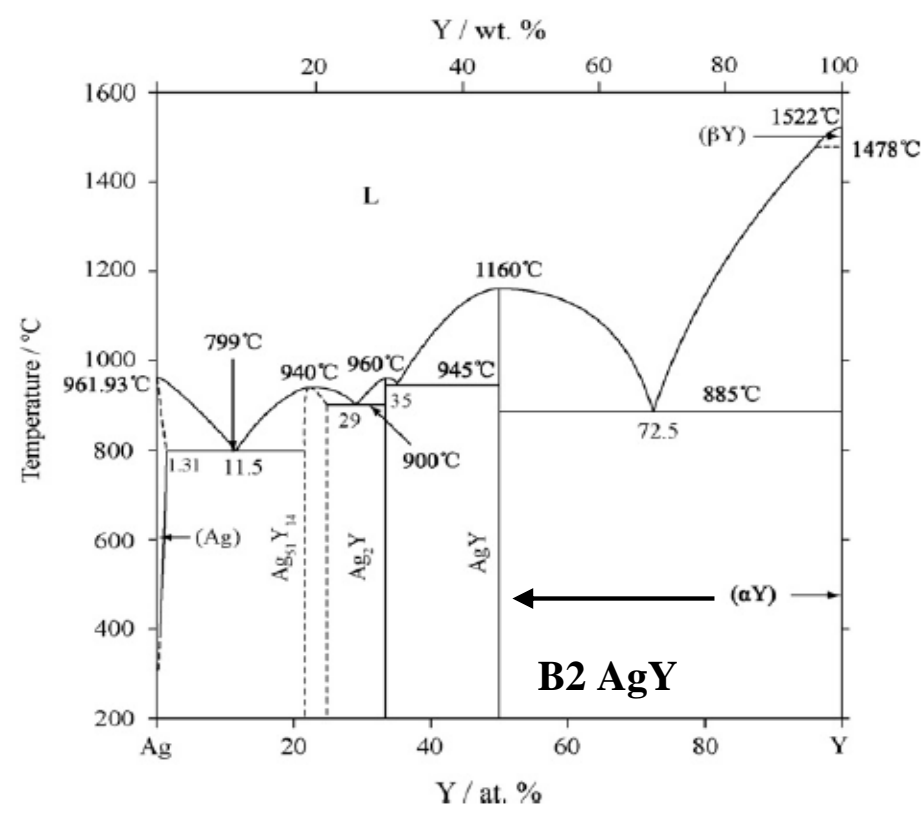

Figure 1.2: Phase diagram of the Ag-Y system showing the B2 AgY compound at 5050 at\% composition [19]. Reprinted with permission of ASM intermational. 


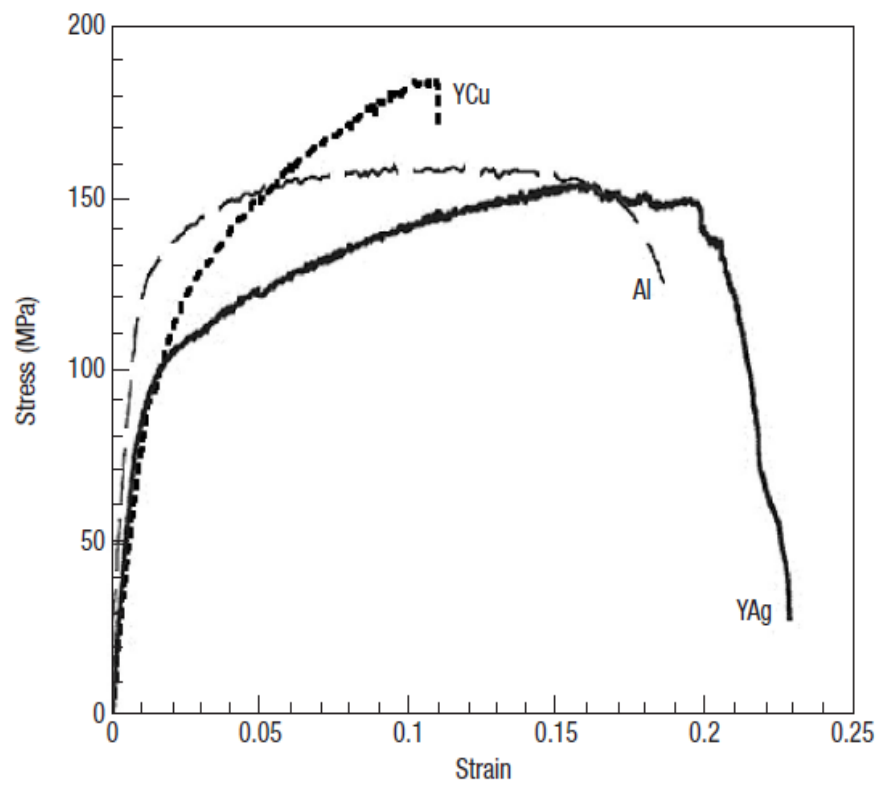

Figure 1.3: Stress- strain curves of some of the ductile MR compounds. Reprinted by permission from Macmillan Publishers Ltd: [Nature Materials] (9), copyright (2003)

Single crystal slip trace analyses performed on $\mathrm{AgY}$ have shown the slip systems to be $\langle 100>\{011\}$ and $<100>\{001\}$, without any evidence of slip in the $<111>$ direction [20]. The a/2 <111>\{110 $\}$ APB energy of AgY has also been shown to be high enough $\left(745 \mathrm{~mJ} / \mathrm{m}^{2}\right.$ [20], $725-880 \mathrm{~mJ} / \mathrm{m}^{2}$ [21]) to prevent the dissociation of the $<111>$ dislocations. TEM analyses carried out on these samples using the $\mathbf{g} \cdot \mathbf{b}$ contrast analysis method unambiguously identified the presence of $<100>$ dislocations [9, 20]. Uncertainty surrounds the meager detection of $\langle 111\rangle$ and $\langle 110\rangle$ dislocations; it is possible that they may be formed due to the junction of $\langle 100\rangle$ dislocations. Furthermore, single crystals oriented with their tensile axis along the $\langle 100\rangle$ direction showed no plasticity and began to crack at stresses of around $300 \mathrm{MPa}$. Additionally, a recent in-situ neutron diffraction study [22] combined with elasto plastic self- consistent modeling (EPSC) has shown that 
the internal elastic strain evolution in ductile $\mathrm{AgCe}$ is nearly identical to that of $\mathrm{NiAl}$ and generally supports the previous observations of dominant $<001>\{110\}$ slip activity.

However, it is to be noted that in polycrystalline metals large stress concentrations may build up at the grain boundaries due to incompatibility and dislocation pile-ups, and these stress concentrations may be of a sufficient magnitude to nucleate slip on the secondary system [23]. Hence, it may be possible to activate the secondary slip system in a polycrystalline specimen but not in a single crystal. This has been observed in ceramics like $\mathrm{LiF}$ and $\mathrm{MgO}[24,25]$.

More than 100 B2 MR phases exist, many of which have been shown to be ductile (e.g., AgY, CuY, CuDy, etc.). Based on this, Gschneidner et al. [9] have speculated that several of the MR compounds may be ductile (Fig.1.4). The present study examines the fracture behavior of MgR compounds which will help in evaluating if this "ductilizing effect" is spread over a large number of B2 rare-earth compounds rather than being restricted to a selectively smaller group.

Similarly, ductility has been reported in group IV refractory metals (R) combined with cobalt (M). The presence of limited polycrystalline ductility in CoZr was first reported in the 1970's [26, 27]. However, it was only in 2005 that tensile elongation as high as $7.5 \%$ was reported [28]. CoZr and CoTi have been studied in detail by several researchers to examine their abnormal ductility [28-30] as well as an anomaly in their yield strength [31-33]. The questions raised by the anomalous ductility in CoZr and CoTi are very similar to those raised by the ductility in MR compounds. 


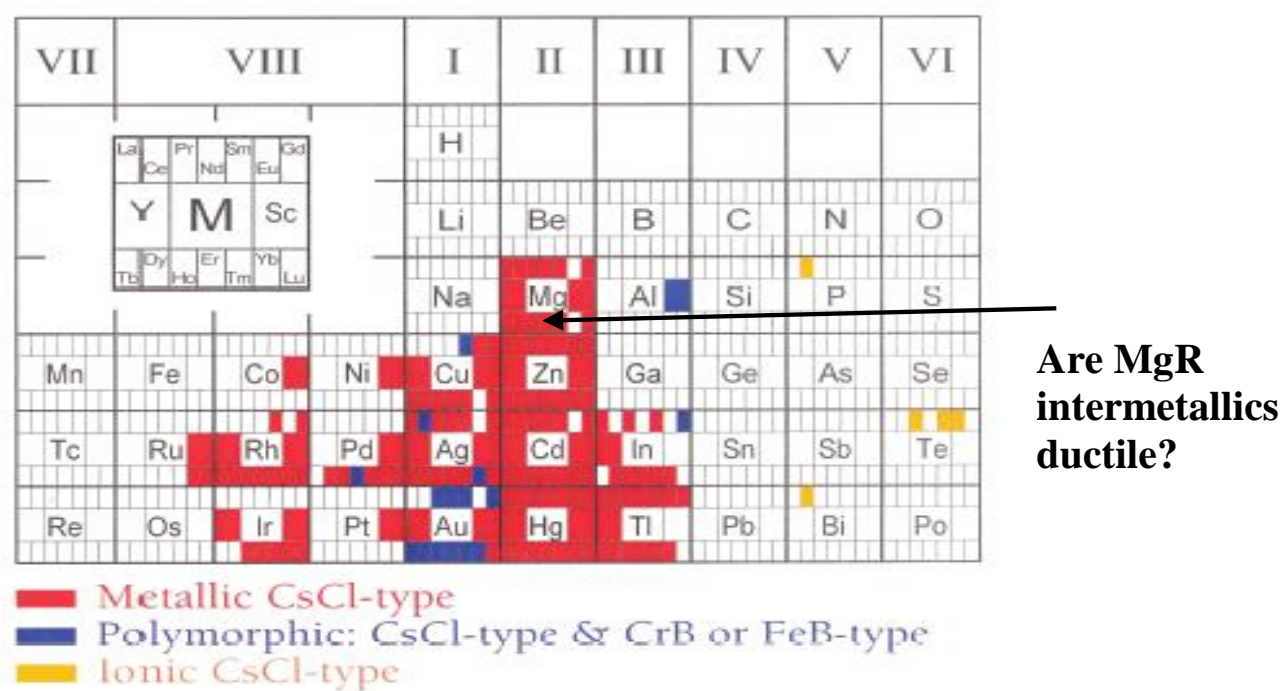

Figure 1.4: A diagram showing the existing B2 MR compounds. The present work examines the ductility in MgR intermetallics. Reprinted by permission from

\section{Macmillan Publishers Ltd: [Nature Materials] (9), copyright (2003)}

TEM analysis of CoZr has only shown the presence of <100> dislocations [34]. $<110>$ dislocations have been seen [35], but are thought to be a result of the junction of $<100>$ dislocations. Single crystal slip trace analysis and TEM measurements carried out on CoTi have been able to detect the presence of only the $<001>\{110\}$ slip systems $[36$, 37]. Among the ductile B2 intermetallic compounds observed, the CoR intermetallics are unique in the fact that $\langle 111\rangle$ dislocations have never been observed by any of the researchers in the samples deformed at room temperature [38].

Recently, an in-situ neutron diffraction study combined with elasto plastic selfconsistent modeling (EPSC) of CoTi and CoZr [39] reported a transition in the strain hardening behavior at approximately $-250 \mathrm{MPa}$ for $\mathrm{CoZr}$ and $-350 \mathrm{MPa}$ for CoTi (Fig.1.5) tested in monotonic compression at room temperature. Prior to this transition, 
only the $<001>\{110\}$ slip mode is active, providing 3 independent slip systems. Beyond the transition, new deformation mechanisms are activated, which permits the grains to undergo arbitrary strains, as would 5 independent slip systems. The outstanding question of this study is related to the exact nature of the new deformation mechanism activated (polycrystal plasticity modeling showed that either $<111>\{110\}$ or $<110>\{110\}$ type slip would comport with the experimental observations.).

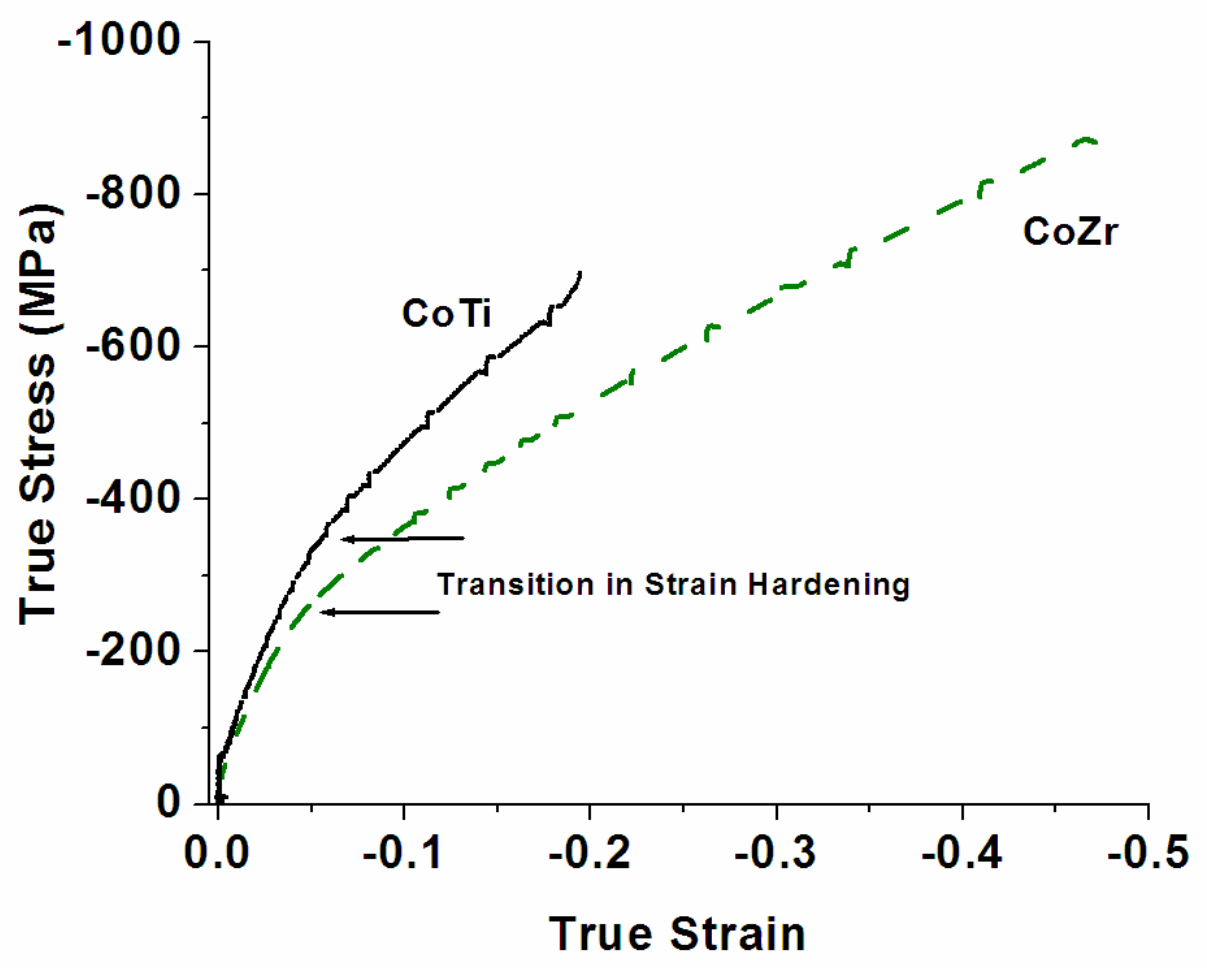

Figure 1.5: Stress- Strain curves for CoZr and CoTi showing the transition in strain hardening. With kind permission from Springer Science+Business Media [39].

Several extrinsic factors are known to the affect the intrinsic strength and ductility of B2 compounds [6]. An increase in ductility in intermetallic compounds may occur in the presence of external contrivances like non-stoichiometry and metastable disorder [38, 
40], special testing environment [41], single crystal specimens [42, 43], addition of a dopant [44], and occurrence of a phase transformation [45]. In the present case, the ductility is observed in binary polycrystalline specimens tested in air. However, the presence of second phases and impurities can even cause intrinsically ductile materials to exhibit a brittle behavior [46, 47]. Furthermore, tensile ductility does not always require the presence of five independent slip systems; the presence of strong crystallographic textures may allow grain boundary cohesion to be maintained with less than five independent slip systems, as has been observed in the case of hexagonal close packed Be [48]. Previous studies have also suggested that a strong texture can influence the tensile ductility of $\mathrm{NiAl}[49,50]$. Additionally, phenomena like twinning [51] and martensitic phase transformations (as seen in pseudoelastic B2 NiTi $[45,52]$ ) provide alternative strain accommodation modes, alleviating the requirement to fulfill von Mises criterion. Such a transformation has been reported in $\mathrm{Co}_{40} \mathrm{Ni}_{10} \mathrm{Zr}_{50}$ and $\mathrm{CoZr}$ [35]. The proposed work will investigate the role of these factors on the ductility observed in the MR compounds.

\subsubsection{The Bauschinger effect}

Many of the B2 intermetallics examined in this study exhibit a large Bauschinger effect. The Bauschinger effect is so large that the material begins to yield in the reverse direction while unloading, i.e., without the application of reverse load. The Bauschinger effect, first reported in 1886 [53], is primarily concerned with an observed reduction in yield strength during reverse loading following prestraining (Fig.1.6). 


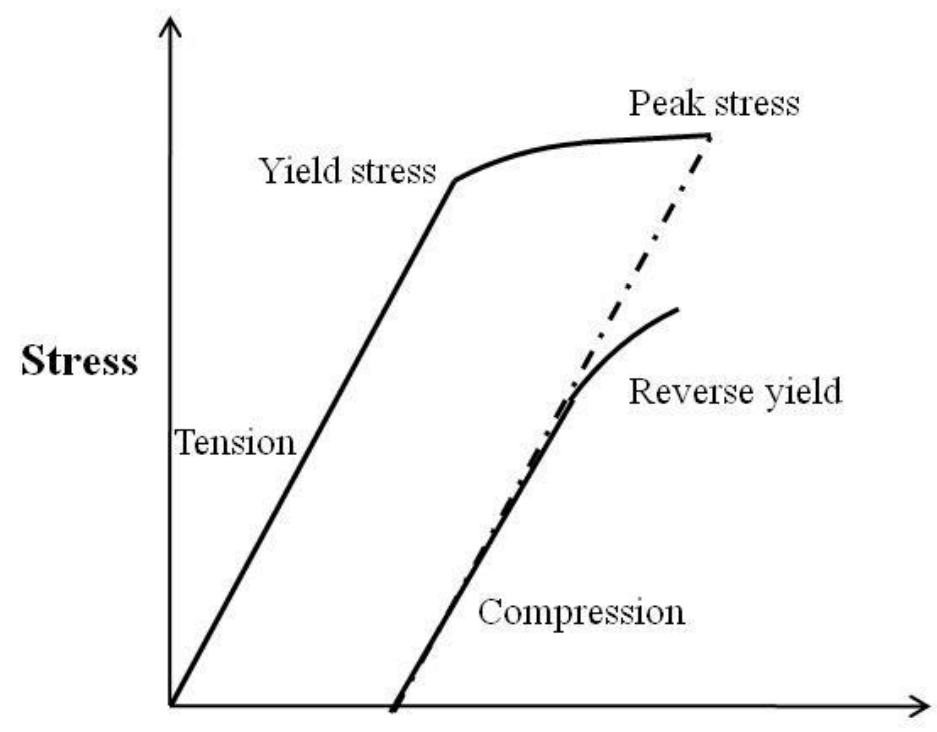

Strain

Figure 1.6: The Bauschinger effect

Its origin was initially attributed to residual stresses due to inhomogeneous deformation of individual grains (intergranular stresses) in polycrystalline materials [5456]. An example is shown in Figure 1.7. If two grains in a sample are oriented such that one is still elastic past the macroscopic yield (grain 1) while one is plastic (grain 2), then on unloading such a sample which was being strained in tension, grain 1 will try to recover its original length. However, grain 2 will contract along the dashed line, leaving it under residual compression. When a reverse load (in this case compressive) is applied to this sample, the residual compressive stresses will assist in dislocation motion and lower the macroscopic yield. 


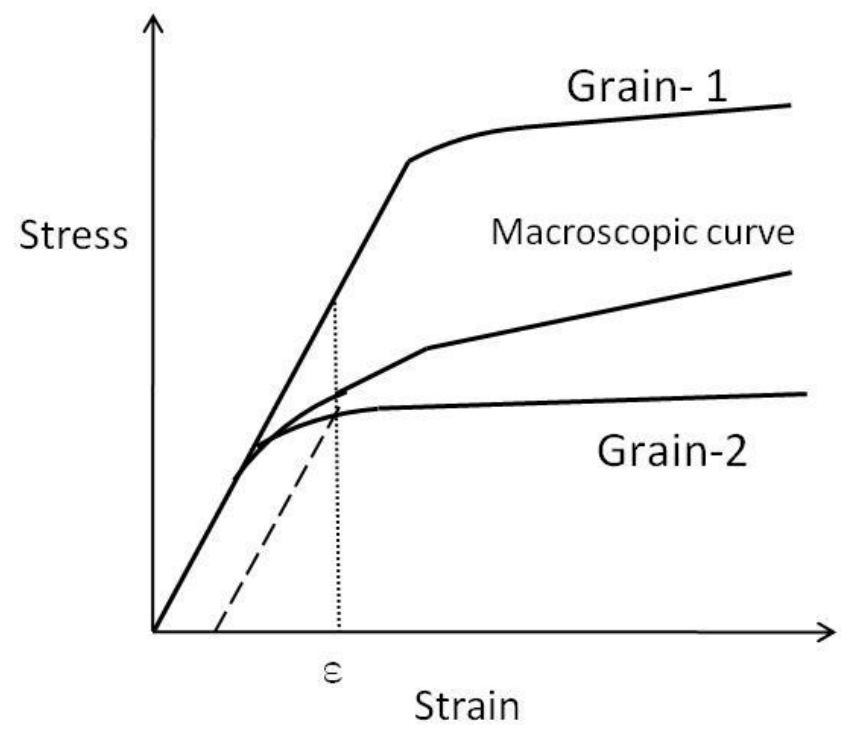

Figure 1.7: Intergranular stresses can cause the Bauschinger effect

However, the observance of the Bauschinger effect in single crystals provided evidence for dislocation-based explanations (intergranular stresses) [57, 58]. It was proposed that back-stresses created by dislocation pile-ups aid dislocation motion in the reverse direction [59] (Fig.1.8). Orowan [60] suggested an alternative dislocation-based theory, centered on a directional resistance to dislocation motion. According to this theory, dislocation motion will be impeded when the obstacles are sufficiently strong. On the removal of applied stress, the dislocation may adjust itself locally, but will remain more or less in the same place. However, when a reverse stress is applied, the row of obstacles in the rear of the dislocation being weaker, will cause reverse yielding to occur at a lower stress. Experiments were carried out to test these theories [61-63] and features of both dislocation-based theories were observed. Later research has revealed that dislocation-based internal stresses are also present in dislocation wall and cell structures, which are formed due to heterogeneous distribution of dislocations, and have been shown 
to be present under applied stress as well as in the unloaded states [64-67]. In spite of the extensive research carried out, the details relating to the Bauschinger effect are still debated, more than 100 years after its discovery.

Dislocation pileups can cause intragranular stresses
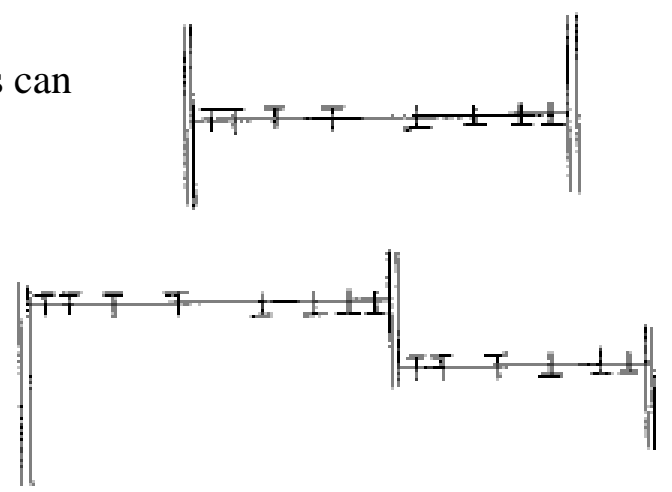

\section{Figure 1.8: Intragranular stresses can be responsible for the Bauschinger effect}

$\mathrm{CoZr}$ and CoTi seem to be ideal candidates to parse the contribution of intergranular stresses to the Bauschinger effect due to the possible change in the number of independent deformation modes past the transition in their strain hardening behavior. As mentioned above, a transition in the plastic deformation occurs in $\mathrm{B} 2 \mathrm{CoTi}$ and $\mathrm{CoZr}$ (Fig.1.5) [39]. As only 3 independent deformation modes are believed to be active prior to this transition, large intergranular stresses are expected to develop. On the other hand, a reduction in the intergranular stresses would be expected, past the transition. 


\subsection{Dissertation Objectives}

Based on the above background the objectives of the present dissertation are:

1. To elucidate the reason for anomalous ductility in select B2 alloys

- Examine the effect of texture, phase purity and order on the anomalous ductility using XRD.

- Study the active deformation modes using TEM and EBSD.

2. To examine the spread of this ductility over the B2 family

- Explore the possibility that $\mathrm{B} 2 \mathrm{MgR}$ intermetallics are ductile by producing, characterizing and testing them.

3. To investigate the large Bauschinger effect shown by these B2 alloys

- Examine the specific contributions of intergranular and intragranular stresses to the Bauschinger effect using in-situ neutron diffraction combined with crystal plasticity modeling.

\subsection{Dissertation format}

Chapters 2-5 correspond to individual journal papers. Suitable modifications have been made to the experimental procedure sections to include relevant details that are usually not covered in journal papers. In some cases, suitable references regarding the experimental procedures have also been added. Chapter 2 investigates the effect of factors like texture, phase purity and order on the ductility of MR alloys using X-ray diffraction. Of the anomalously ductile alloys, CoZr and CoTi have been studied by several groups and parameters, such as electropolishing conditions which are required for sample preparation, are documented. Hence, the focus is on CoTi in chapter 3. Chapter 3 
explores the slip modes present in the anomalously ductile CoTi, particularly at higher strains using electron backscattered diffraction (EBSD) and transmission electron microscopy (TEM). Chapter 4 examines the spread of ductility over the B2 family by examining the deformation and fracture behavior of $\mathrm{MgR}$ intermetallics. Chapter 5 explores the Bauschinger effect in $\mathrm{B} 2 \mathrm{CoZr}$ using in-situ neutron diffraction combined with elasto plastic self-consistent modeling. CoZr was chosen for this study over CoTi because the negative neutron scattering cross-section of $\mathrm{Ti}$ increases the time needed to collect a single diffraction pattern from CoTi by a factor of 4 as compared to CoZr. Chapter 6 discusses the summary and overall conclusions of this thesis. Finally, chapter 7 considers avenues for future work.

\subsection{References}

1. Sauthoff G. Intermetallics, New York: VCH; 1995.p.1-5.

2. Lasalmonie A. Intermetallics 2006; 14(10-11):1123-1129.

3. Ball A and Smallman RE. Acta Metallurgica 1966; 14(10):1349-1355.

4. Ball A and Smallman RE. Acta Metallurgica 1966; 14(11):1517-1526.

5. Takasugi T and Yoshida M. Phil. Magazine Letters 1995; 72(5):303-310.

6. Baker I. Deformation \& Fracture of strongly-ordered B2 compounds in Deformation and fracture of ordered intermetallic materials III 1996; Warrendale, PA: TMS (The Minerals, Metals \& Materials Society):27-44

7. von Mises R, Z. Ang. Math. Mech. 1928;8,161-185

8. Kelly A and Macmillan NH, Strong solids 3rd edition 1986; Clarendon Press, Oxford : 116-117. 
9. Gschneiner K, et al. Nature Mater. 2003; 2:587-590

10. Kaneno Y, et al. J. of Alloys and Compounds 2008; 456(1-2): 125-34.

11. Baker I. Materials Science and Engineering A 1995; 193:1-13.

12. Noebe RD, Bowman RR and Nathal MV. International Materials Reviews 1993; 38(4): 193-232.

13. Miracle DB. Acta Metallurgica et Materialia 1993; 41(3): 649-684.

14. Fu CL, Yoo MH. First-Principles Investigation of Mechanical Behavior of B2 Type Aluminides: FeAl and NiAl, in: Johnson LA, et al. (Eds.). HighTemperature Ordered Intermetallic Alloys IV. Pittsburgh, PA: MRS (Materials Research Society); 1991.p. 667-72.

15. Veyssiere P and Noebe R. Philosophical Magazine A 1992; 65(1):1-13.

16. Potter DI. Materials Science and Engineering 1970; 5(4): 201.

17. Rachinger WA and Cottrell AH. Acta Metallurgica 1956; 4(2):109-113.

18. Saka H and Kawase M. Philosophical Magazine A 1984; 49(4):525-533.

19. Gschneidner K, Calderwood FW. Ag-Y, in: Massalski TB. (Eds.). Binary Alloy Phase Diagrams $2^{\text {nd }}$ Edition Vol.1 ASM International: Materials Park, Ohio; 1983. p. 114

20. Russell AM, et al. Acta Materialia 2004; 52(13): 4033-4040.

21. Wu YR, Hu WY, and Han SC. Physica B-Condensed Matter 2008; 403(19-20): 3792-3797.

22. Wollmershauser JA, Kabra S, and Agnew SR. Acta Materialia 2009; 57(1):213223.

23. Evans AG and Langdon TG. Progress in Material Science 1976;21:242 
24. Scott WD and Pask JA, J. American Ceramic Society 1963; 46: 284.

25. Langdon TG and Pask JA, J. American Ceramic Society 1971; 54: 240.

26. Hossain D, Harris IR, and Barraclough KG. Journal of the Less Common Metals 1974; 37(1): 35-57.

27. Lall C, Loretto MH, and Harris IR. Acta Metallurgica 1978; 26(10): 1631-1641.

28. Yamaguchi T, Kaneno Y, and Takasugi T. Scripta Materialia 2005; 52(1): 39-44.

29. Takasugi T. and Izumi O. Journal of Materials Science 1988; 23(4): 1265-1273.

30. Kaneno Y. and Takasugi T. Journal of Materials Science 2003; 38(5): 869-876.

31. Takasugi T, Izumi O, and Yoshida M. Journal of Materials Science 1991; 26(11): 2941-2948.

32. Takasugi T, et al. Philosophical Magazine A 1995; 71(2):347 - 358.

33. Wittmann M and Baker I. Materials Science and Engineering A 2002; 329-331: 206-212.

34. Yoshida M and Takasugi T. Philosophical Magazine A 1993; 68(2):401-417.

35. Francois A and Veyssiere P. Intermetallics 1994; 2(1): 9-22.

36. Takasugi T, et al. Philosophical Magazine A 1990; 61(5):785 - 800.

37. Takasugi T, Yoshida M, and Kawabata T. Philosophical Magazine A 1992; 65(1): $29-40$.

38. Russell A. Ductile, Stoichiometric B2 Intermetallics, in Intermetallics Research Progress 2008; Nova Science Publishers: New York: 213-235.

39. Wollmershauser JA, Neil CJ, and Agnew SR. Metallurgical and Materials Transactions A 2010; 41A(5): 1217-1229.

40. Takasugi T, Masahashi N, and Izumi O. Acta Metallurgica 1987; 35(2): 381-391. 
41. Liu CT. Scripta Metallurgica Et Materialia 1992;27(1): 25-28.

42. Levit VI, et al. Scripta Materialia 1996;34(12): 1925-1930.

43. Ebrahimi F and Shrivastava S. Acta Materialia 1998;46(5):1493-1502.

44. Ishida K, et al. Metallurgical Transactions A 1991;22(2): 441-446.

45. Huang XY, Ackland GJ and Rabe KM. Nature Materials 2003; 2(5): 307-311.

46. Wood DL, Westbrook JH, J.Nucl. Mater. 1964;12:208

47. Westbrook JH, Metallurgical Reviews. 1964;9:415

48. Webster D, Beryllium science and technology, vol. 1 1979;New York: Plenum Press:133.

49. Rozner AG, Wasilewski RJ, Journal of institute of metals 1966;94:169

50. Hahn KH, Vedula K, Scripta Metall. 1989;23:7-12

51. Kocks UF and Westlake DG. Trans. AIME 1967;239: 1107-09.

52. Vaidyanathan R, Bourke MAM, and Dunand DC. Materials Science and Engineering A 1999; 273-275: 404-409.

53. Bauschinger J. Mitt.Mech.-tech Laboratorium K. Technischen Hochschule Munchen 1886;13(5):31.

54. Heyn E. Metall und Erz 1918; 22: 441-2 and 436-41.

55. Thompson N, Wadsworth NJ. Advances in Physics 1958; 7: 72.

56. Polakowski NH and Ripling EJ. Strength and Structure of Engineering materials 1966; Prentice and Hall, Englewood Cliffs, NJ: 144-146.

57. Gough HJ, Wrights SJ and Hanson D. Aeronautical Res. Ctttee. 1924; R. and M. No.995.

58. Sachs G and Shoji H. Z fur Physik 1927; 45: 775. 
59. Zener C. Elasticity and Anelasticity of Metals 1948; The University of Chicago Press, Chicago, IL: 145-146.

60. Orowan E. Causes and Effects of Internal Stresses in Internal Stresses and Fatigue in Metals symposium 1958: Elsevier, London; 59-80.

61. Wu TM. Investigation of the Bauschinger effect in metals 1958:M.I.T Master's Thesis.

62. Canal JR. Investigation of the Bauschinger effect in Copper 1960:M.I.T Master's Thesis.

63. Deak GI. A study on the caues of the Bauschinger effect 1962:M.I.T Doctoral Thesis.

64. Seeger A and Wilkens M. in Realstruktur und Eigenschaften on Reinststoffen (edited by E. Rexer) Vol. 3 1967; Akademie, Berlin: 31.

65. Grosskreutz JC and Mughrabi H.in Constitutive Equations in Plasticity (edited by A. S. Argon) 1975; MIT Press, Cambridge, MA:251.

66. Mughrabi H, Ackermann F and Herz K. in Fatigue Mechanisms, ASTM STP 615 (edited by J. T. Fong) 1979: ASTM, Philadelphia, PA:.69.

67. Mughrabi H. Acta Metallurgica 1983; 31(9): 1367-1379. 


\title{
2. X-ray diffraction study of the phase purity, order, and texture of ductile B2 intermetallics ${ }^{i}$
}

\author{
R.P. Mulay ${ }^{\text {a }}$, J.A. Wollmershauser ${ }^{\text {a }}$, M.A. Heisel ${ }^{\text {a }}$, H. Bei ${ }^{\mathrm{b}}$, A.M. Russell ${ }^{\text {c }}$ and \\ S.R.Agnew ${ }^{\text {a }}$ \\ ${ }^{a}$ Materials Science and Engineering, University of Virginia, \\ Charlottesville, VA 22904-4745 \\ ${ }^{\mathrm{b}}$ Oak Ridge National Laboratory, Material Science \& Technology Division, \\ Oak Ridge, TN 37831 \\ ${ }^{\mathrm{c}}$ Iowa State University, Department of Materials Science \& Engineering,
} Ames, IA 50011

\subsection{Abstract}

Representatives (AgY, CuY, AgEr, CuDy, MgY and $\mathrm{MgCe}$ ) of the newly discovered family of ductile stoichiometric B2 intermetallic (metal - rare-earth element, MR) compounds are characterized by $\mathrm{x}$-ray diffraction, to determine if their anomalous ductility is related to an exceptional level of phase purity, lack of chemical ordering, or a strong crystallographic texture. Brittle NiAl serves as an anti-type in this study. We found that all of the rare-earth compounds, except $\mathrm{MgY}$, have a significant volume fraction ( $\sim-20 \mathrm{vol} \%)$ of second phases $\left(\mathrm{M}_{2} \mathrm{R}\right.$ intermetallics and $\mathrm{R}_{2} \mathrm{O}_{3}$ oxides), which has not been reported in previous studies of these materials. The most ductile of observed

\footnotetext{
${ }^{\text {i }}$ R. P. Mulay, et al. "X-ray diffraction study of the phase purity, order and texture of ductile B2 intermetallics." Acta Materialia 2010; 58(7): 2788-2796. Reproduced with permission from Elsevier.
} 
MR compounds, AgY, is highly ordered. A moderate texture is observed in $\mathrm{AgY}$, which may explain its higher ductility (using polycrystal modeling) as compared to other MR compounds. However, the intrinsic polycrystalline ductility of these compounds in the randomly textured state (like that observed in $\mathrm{CuY}$ ) still has no specific, definitive explanation.

Keywords: ductility, intermetallics, ordering, preferred orientation, dislocations

\subsection{Introduction}

A family of ductile intermetallic compounds has been recently discovered [1]. The main characteristics, identified in previous publications, are that they are fullyordered, stoichiometric line compounds composed of a simple or transition metal (M) combined with a rare-earth metal $(\mathrm{R})$, and that they have a $\mathrm{B} 2(\mathrm{CsCl})$ crystal structure. The most ductile compound observed to date is $\mathrm{AgY}$, which has been reported to exhibit a tensile ductility of more than $20 \%$. CuY and $\mathrm{CuDy}$ have also shown significant ductility, and more than 100 other B2 MR phases exist, many of which could be ductile [1]. However, some of the MR phases have already been shown to be brittle [2,3]. For example, the present authors have studied a series of $\mathrm{MgR}$ compounds, and it has been shown that many are brittle ${ }^{\mathrm{ii}}$, exhibiting cleavage type fracture [2].

Other ductile B2 intermetallics, such as AuZn, AgMg and $\mathrm{CuZn}$, have been shown to deform much in the same way as BCC metals, i.e., by the slip of dislocations with $<111>$ direction Burgers vectors $[4,5]$ and this permits them to satisfy the von Mises

\footnotetext{
${ }^{\text {ii }} \mathrm{MgY}$ may be an exception as it as shown about $10 \%$ ductility in compression [3,4] and moderate ductility $(\sim 2 \%)$ in tension [4]. However, several instances of brittle fracture have been observed in castings (i.e., before being extracted from their molds) and in general handling of this material $[3,4]$.
} 
criterion [6] for homogeneous polycrystalline ductility, which requires 5 independent slip systems. Low anti-phase domain boundary (APB) energy is generally held to be a prerequisite for $<111>$ slip to operate in the B2 structure at low homologous temperatures [4,12] as is seen in $\mathrm{CuZn}[7,8]$.

Single crystal slip trace analyses performed on $\mathrm{AgY}$ have shown the slip systems to be $\langle 100\rangle\{011\}$ and $\langle 100\rangle\{001\}$, without any evidence of slip in the $<111>$ direction [9]. The APB energy of AgY has also been shown to be high enough to prevent the dissociation of the $\langle 111>$ dislocations $[9,10]$. Such compounds, with limited slip, have traditionally exhibited significant ductility only when a special testing environment like vacuum or oxygen or special material state exists, such as non-stoichiometry [e.g.11], metastable disorder [e.g.12], doping [e.g.13], etc. Yet the ductility observed in the present MR compounds appears to have been obtained without any of these "extrinsic" contrivances. This has been one of the most surprising features of this discovery, namely, that these compounds seem to behave in violation of the von Mises criterion.

No experiments have been reported regarding the role that phase purity, ordering and texture may play in the ductility of these MR compounds. It is noted that the strength and ductility of B2 compounds is affected by many extrinsic factors, one of which is the impurity content [14]. In fact, the presence of second phases and impurities can even cause intrinsically ductile materials to exhibit a brittle behavior [e.g. 15, 16]. As mentioned above, it has been found that metastable disorder can lead to an increase in ductility. However, to the authors' knowledge, no studies have been carried out to quantitatively determine the order parameter of these compounds. It is noted that tensile ductility does not always require the presence of five independent slip systems, because 
the presence of strong crystallographic textures may allow grain boundary cohesion to be maintained with less than five independent slip systems, as has been observed in the case of textured hexagonal close packed Be [17]. Perhaps more pointedly, it has been suggested that a strong texture can influence the tensile ductility of NiAl [18, 19]. Finally, Baker and Schulson [20] have proposed the possibility that "diffusion assisted restorative processes," including dynamic recovery and recrystallization, may be responsible for ductility in $\mathrm{B} 2 \mathrm{NiAl}$ deformed at a homologous temperature of around 0.4. However, in case of $\mathrm{AgY}$, the ductility observed is at a low homologous temperature of 0.2 and hence the possibility of diffusional accommodation is viewed as unlikely. We presently examine phase purity, order and crystallographic texture of compounds from the newly discovered family using x-ray diffraction.

\subsection{Experimental Procedures}

\subsubsection{Alloy Preparation}

Transition Metal- Based Intermetallics: $\mathrm{AgY}, \mathrm{AgEr}, \mathrm{CuY}$ and $\mathrm{CuDy}$ were made at the Ames Laboratory [21]. The same heats of these materials have been tested and proven to be ductile by the group at Ames [1]. The details of the method of preparation of these alloys were published previously [22]. An important point to note is that these materials have been encapsulated in stainless steel and hot rolled. Another AgY alloy sample, used for the order parameter determination, was prepared at the University of Virginia (UVa) from high purity Ag (99.9 wt \%) and Y (99 wt \%) by arc melting in an Ar atmosphere. Yet another AgY alloy sample was arc melted and drop cast at Oak Ridge National Laboratory (ORNL). 
A brief description of the arc melting procedure:

In the arc melter, the raw materials are placed in a water cooled copper hearth. The hearth is also equipped with an insert in which titanium is placed. Initially the entire chamber is pumped down from atmospheric pressure (with a cold diffusion pump) using a roughing pump. In this configuration, the main roughing valve is closed and the foreline and gate valves are open. When the pressure in the chamber is below $6.67 \mathrm{~Pa}$, the diffusion pump is turned on. When the pressure in the chamber is below $6.67 \times 10^{-4} \mathrm{~Pa}$, the gate valve is closed, the diffusion pump is turned off and the chamber is back-filled with with argon to a pressure of $30 \mathrm{kPa}$. The foreline valve should be kept open and the roughing pump must remain on. Once the chamber has been back-filled with argon an arc is struck between the tungsten electrode and the titanium. Since titanium has a high affinity for oxygen it serves as a "getter" of oxygen remaining in the chamber. Once the titanium has melted completely, the arc is shifted to the charge which is then melted. The charge may be melted and flipped several times to ensure homogeneity of the melt.

Magnesium based intermetallics: The MgY castings were prepared using high-purity Mg (99.9 wt \%) and Y (99 wt \%) at ORNL. The alloying was performed using a Pt-wire resistive furnace within an inert-gas (Ar) filled glove box with additional oxidation protection provided by small additions of the heavy cover gas, $\mathrm{SF}_{6}$. The $\mathrm{Mg}$ was melted within a mild steel crucible coated with Zircwash $\left(\mathrm{ZrO}_{2}\right)$ and the $\mathrm{Y}$ was then added to the melt. The molten metal was cast into a cylindrical copper mold yielding castings of $\sim 12$ mm x $100 \mathrm{~mm}$. The MgCe alloy was prepared at UVa from high-purity Mg (99.9 wt \%) 
and Ce (99.5 wt \%) using induction melting in an evacuated chamber, back-filled with Ar. Both the alloying and solidification were carried out in a graphite crucible coated with boron nitride. Finally, the NiAl sample was arc melted, alloyed, and drop cast into a chilled $\mathrm{Cu}$ mold $\sim 12 \mathrm{~mm} \times 100 \mathrm{~mm}$ within an Ar atmosphere at ORNL.

A brief description of the induction melting procedure:

The raw materials are placed in a graphite crucible coated with boron nitride. The coated crucible should be baked out at around $150^{\circ} \mathrm{C}$ for 1 hour prior to being used. The chamber is evacuated using a roughing pump to a pressure of around $1.33 \mathrm{~Pa}$ and it is then back-filled with argon to a pressure of $30 \mathrm{kPa}$.

Details regarding the purity of the alloys prepared at Ames Laboratory can be found elsewhere [1]. The chemical compositions of all the other alloys were determined by Northern Analytical using the inductively coupled plasma mass spectrometry (ICPMS) technique. The LECO combustion technique was used for determining the carbon and oxygen contents. In atomic percent, the compositions were $49.3 \% \mathrm{Mg} / 50.4$ \% Y for $\mathrm{MgY} ; 50.1 \% \mathrm{Mg} / 49.8 \% \mathrm{Ce}$ for $\mathrm{MgCe} ; 49.4 \% \mathrm{Ni} / 50.5 \% \mathrm{Al}$ for $\mathrm{NiAl}$; and 52.1 $\% \mathrm{Ag} / 47.1 \% \mathrm{Y}$ for $\mathrm{AgY}$. After considering the assays of the raw materials and likely additional impurities dependent on the method of melting, selected impurities were determined and their amounts are given in Table 2.1. 
Table 2.1: Impurities analyzed in $\mathrm{AgY}, \mathrm{MgY}, \mathrm{MgCe}$ and $\mathrm{NiAl}$ in atomic percent $\times 10^{2}$

\begin{tabular}{|c|c|c|c|c|c|c|c|c|c|c|c|}
\hline At \% & Al & W & Cu & Zr & Mn & B & Fe & Si & Ta & C & O \\
\hline AgY & 0.91 & 4.46 & & & & & & & & & 79.45 \\
\hline MgY & 6.13 & 0.32 & 0.33 & 22.32 & & & & & & & \\
\hline MgCe & 7.02 & & & & 0.42 & 0.69 & & & & & \\
\hline NiAl & & & 0.12 & & & & 0.76 & 1.14 & $<0.01$ & 2.98 & 0.66 \\
\hline
\end{tabular}

\subsubsection{Quantitative Phase Analysis}

The compounds AgY, AgEr, CuY, CuDy, MgY and NiAl were examined for phase purity. X-ray diffraction scans of $2 \theta$ from 20 to $100^{\circ}$ were done on the materials in the bulk form (not powdered) using continuous scans of $1 \%$ min with a step size of $0.05^{\circ}$. The measurements were carried out on a Scintag XDS 2000 diffractometer with a $\mathrm{Cu} \mathrm{K}_{\alpha}$ sealed tube source, and the data obtained were compared to powder diffraction file (PDF) data to determine the phases present. [Slit sizes and samples sizes were chosen such that the size of the incident X-ray beam did not exceed the sample size in the range of $2 \theta$ being examined.] The quantitative phase analysis was carried out using the direct comparison method [23]. The diffracted intensity is given by

$$
I=\frac{K \times R}{2 \mu_{m}}
$$

where, $K$ is a constant independent of the kind and amount of diffracting substance, $\mu_{m}$ is the linear absorption coefficient of the mixture, and $R$ is given by 


$$
R=\frac{m \times L P \times e^{-2 M} \times F^{2}}{v^{2}}
$$

where, $m$ is the multiplicity, $L P$ is the Lorentz Polarization Factor, $\mathrm{e}^{-2 M}$ is the temperature factor, $F$ is the structure factor, and $\mathrm{v}$ is the unit cell volume. If we consider an alloy consisting of two phases $A$ and $B$,

$$
I_{A}=\frac{K \times R_{A} \times C_{A}}{2 \mu_{m}} ; I_{B}=\frac{K \times R_{B} \times C_{B}}{2 \mu_{m}}
$$

where $C_{A}$ and $C_{B}$ are the concentrations in volume percent of phases $A$ and $B$. This gives us,

$$
\frac{I_{A}}{I_{B}}=\frac{R_{A} \times C_{A}}{R_{B} \times C_{B}}
$$

From this equation, the value of $C_{A} / C_{B}$ can be obtained and assuming that these are the only two phases present (i.e. $C_{A}+C_{B}=1$ ) the values of $C_{A}$ and $C_{B}$ (volume fraction) are obtained. The same concept can be extended to more than two phases and in this case has been extended to three phases, i.e. $C_{A}+C_{B}+C_{C}=1$.

\subsubsection{Order Parameter Determination}

The order parameters of the compounds $\mathrm{AgY}, \mathrm{MgY}, \mathrm{MgCe}$ and $\mathrm{NiAl}$ were determined, with the latter serving as a reference, because it was already known to be fully ordered at room temperature [25, 29]. Powder samples were produced from castings by filing. The powder was encapsulated in a narrow quartz tube with $\mathrm{Ti}$ as a getter and annealed for 24 hours and then furnace cooled. $\mathrm{AgY}$ was annealed at a temperature of $625^{\circ} \mathrm{C}, \mathrm{MgY}$ at $500^{\circ} \mathrm{C}, \mathrm{MgCe}$ at $350^{\circ} \mathrm{C}$, and $\mathrm{NiAl}$ at $800^{\circ} \mathrm{C}$. The powder was then sieved to 450 mesh $(<32 \mu \mathrm{m})$. X-ray diffraction was carried out by step scanning with a $2 \theta$ step 
size of $0.01^{\circ}$ over the range of 20 to $120^{\circ}$ with a counting time of $8 \mathrm{~s}$. [Again, Slit sizes and samples sizes were chosen such that the size of the incident X-ray beam did not exceed the sample size in the range of $2 \theta$ being examined.]

The order parameter can be determined by comparing the intensity of a superlattice reflection to that of a fundamental reflection [24]. The experimentally observed intensities were analyzed using the method given below [25]. The integrated intensity of an X-ray peak is given by [23];

$$
I_{0}(h k l)=\left(\frac{K \times m \times L P \times F^{2}}{2 \mu_{m} v^{2}}\right) \times e^{-2 M}
$$

where $M$ depends on the x-ray wavelength and diffraction angle as follows:

$$
M=B\left(\frac{\sin \theta}{\lambda}\right)^{2}
$$

Because we are focusing on a single phase for which multiple peaks are observable, it is, in this case, possible to estimate the temperature factor mentioned above. The unknown factor, $B$, can be determined empirically by linear regression of the following relationship involving the calculated $\left(\mathrm{I}_{0}\right)$ and observed intensity, $I_{o b s}$

$$
\ln \left(\frac{I_{0}}{I_{o b s}}\right)=2 B\left(\frac{\sin \theta}{\lambda}\right)^{2}-\ln C
$$

where $C$ is another empirical parameter. Using the derived value, $B$, we can determine the order parameter, $S$, by comparing the observed intensity ratio with the calculated intensity ratio for the case of a perfectly stoichiometric compound.

$$
S=\sqrt{\frac{I_{o b s}(h k l) / I_{o b s}\left(h^{\prime} k^{\prime} l^{\prime}\right)}{I_{0}(h k l) / I_{0}\left(h^{\prime} k^{\prime} l^{\prime}\right)}}
$$


where $(h k l)$ refers to a superlattice reflection and $\left(h^{\prime} k^{\prime} l^{\prime}\right)$ refers to a fundamental reflection. Because of the $\theta$ dependence in $M$ (see Eq. 6), the temperature factor does not cancel out of the calculated ratio.

\subsubsection{Texture Determination}

A Scintag X1 diffractometer equipped with a four circle goniometer and $\mathrm{Cu} \mathrm{K}_{\alpha}$ sealed tube source was used for crystallographic texture measurements in the Shulz reflection geometry. The (100), (110) and (211) pole figures were measured on a $5 \times 5^{\circ}$ grid for sample tilts $\chi=0-80^{\circ}$ and azimuthal rotations $\phi=0-355^{\circ}$. Defocusing corrections were made using experimentally determined defocusing curves from standard random powder samples. Complete orientation distributions and recalculated full pole figures were determined using the Williams-Imhof-Matthies-Vinel (WIMV) algorithm within the preferred orientation package of Los Alamos (popLA) code [26]. The compounds $\mathrm{AgY}, \mathrm{AgEr}, \mathrm{CuY}$ and $\mathrm{MgY}$ have been examined.

\subsection{Results}

\subsubsection{Phase Content}

The X-ray diffraction patterns for the materials analyzed are shown in Figure 2.1. All of the ductile $\mathrm{B} 2$ alloys show peaks of the $\mathrm{M}_{2} \mathrm{R}$ phase and $\mathrm{R}_{2} \mathrm{O}_{3}$ phase (in addition to the primary MR phase) clearly showing that these materials are not single phase. $\mathrm{MgY}$ (Fig.2.1e) appears to be single phase, to within the resolution of the technique $(\sim 2$ volume \%), with no other peaks being present. The small peak preceding the (110) peak is a $\mathrm{W} \mathrm{L}_{\alpha}$ "escape peak". Similarly, NiAl is also shown (Fig.2.1f) to be single phase. The quantitative phase fractions are shown in Table 2.2. As previously stated, the ductile MR 
intermetallics are line compounds and any deviation from ideal composition (50:50) during alloy preparation will result in second phase formation. In contrast, $\mathrm{NiAl}$ and $\mathrm{MgY}$ are not line compounds and a small deviation from ideal composition will still result in a single phase.

Table 2.2: Quantitative phase fractions (vol. \%)

\begin{tabular}{|c|c|c|c|}
\hline Alloy & MR & $\mathbf{M}_{2} \mathbf{R}$ & $\mathbf{R}_{\mathbf{2}} \mathbf{O}_{\mathbf{3}}$ \\
\hline $\mathrm{AgY}$ & 85.70 & 9.35 & 4.98 \\
\hline $\mathrm{AgEr}$ & 94.30 & 2.84 & 2.85 \\
\hline $\mathrm{CuY}$ & 84.90 & 10.90 & 4.17 \\
\hline $\mathrm{CuDy}$ & 80.53 & 16.02 & 3.44 \\
\hline $\mathrm{MgY}$ & 100.00 & 0.00 & 0.00 \\
\hline $\mathrm{NiAl}$ & 100.00 & 0.00 & 0.00 \\
\hline
\end{tabular}

In our calculations of phase content (using Eq. 4), we have neglected the effect of the temperature factor on the intensities, because only a few reflections are present for the minority phases, making calculation of the temperature factor susceptible to inaccuracies. Because we have also characterized the texture present in the majority phase (discussed below) we have intentionally selected reflections, for the quantitative analysis, so as to minimize the effects of texture. In all cases, except $\mathrm{CuY}$, the (110) reflection has been used for the MR stoichiometric phase. In case of $\mathrm{CuY}$, the texture is almost perfectly 
random and, hence, the (111) reflection was used because the (110) was convoluted with other small peaks which could not be independently resolved. For the $\mathrm{M}_{2} \mathrm{R}$ phase and the $\mathrm{R}_{2} \mathrm{O}_{3}$ phases, the reflections which were clearly resolved and were unique to that particular phase (i.e. the reflection was not overlapping with reflections from other phases) were selected. In case of the $\mathrm{R}_{2} \mathrm{O}_{3}$ phase this is the (222) reflection. For $\mathrm{Ag}_{2} \mathrm{Y}$, $\mathrm{Ag}_{2} \mathrm{Er}$ and $\mathrm{Cu}_{2} \mathrm{Y}$ the (103) reflection was used, while for $\mathrm{Cu}_{2} \mathrm{Dy}$ the (200) reflection was used. Table 2.2 shows the results obtained. 

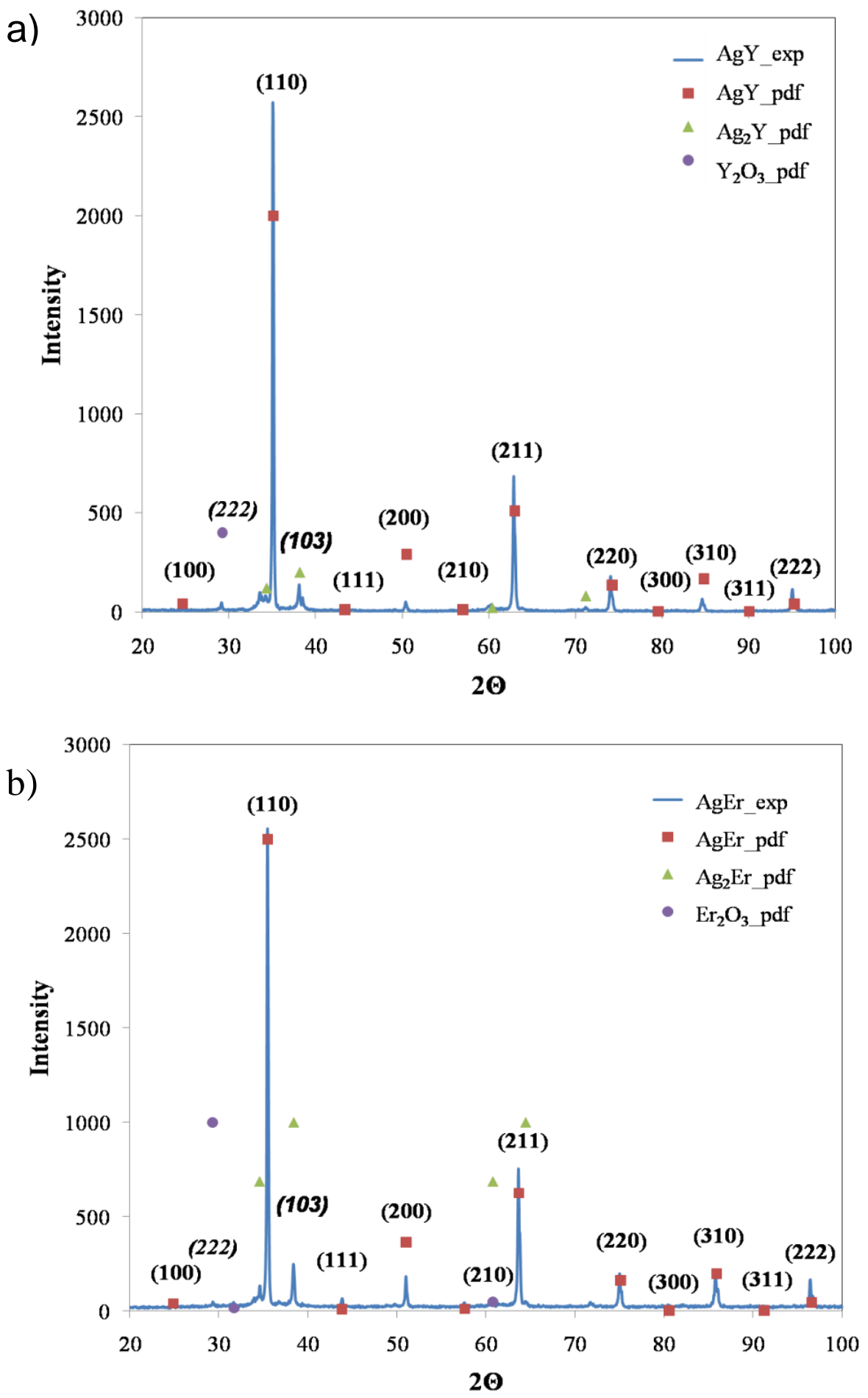

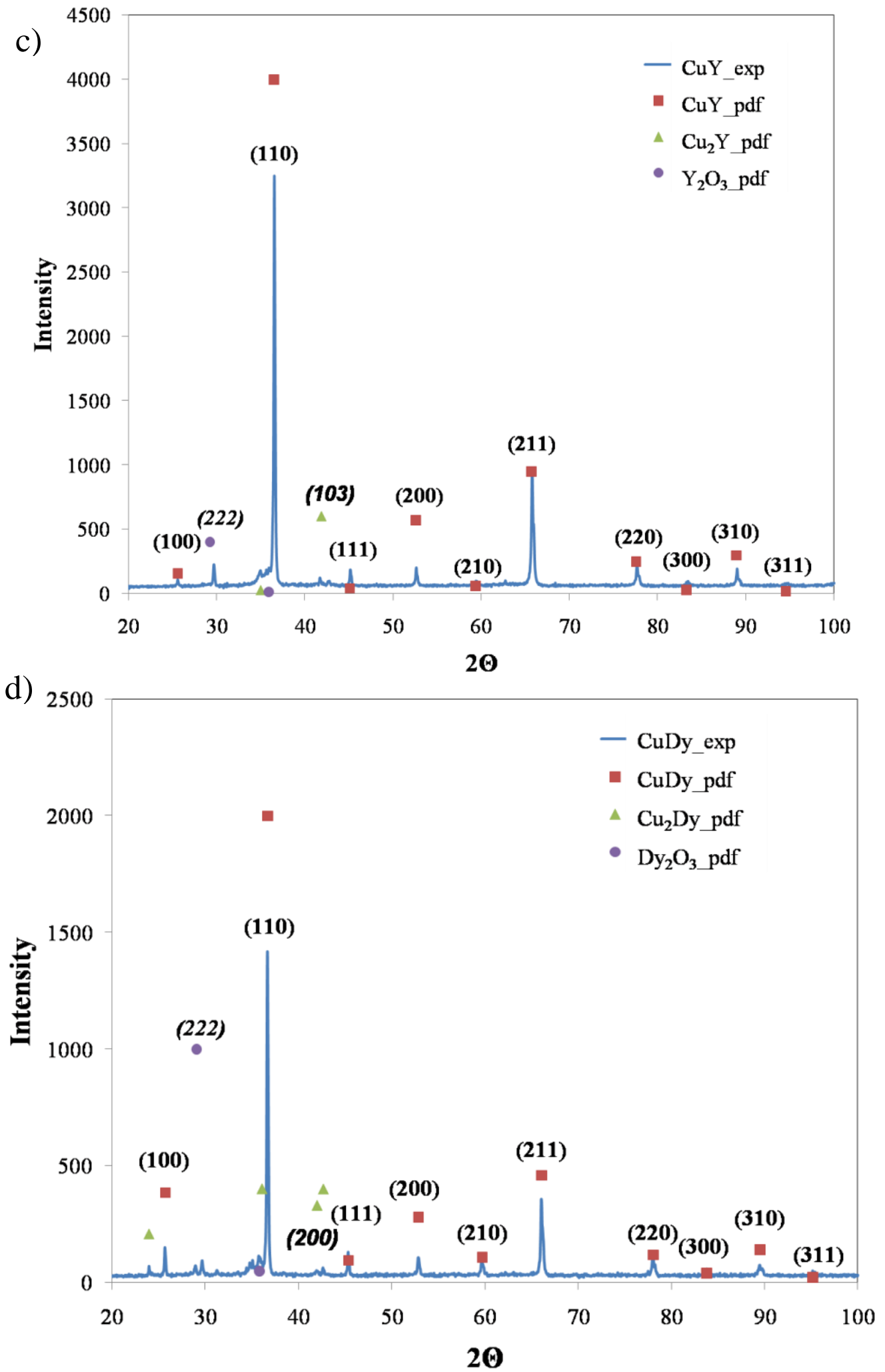
e)

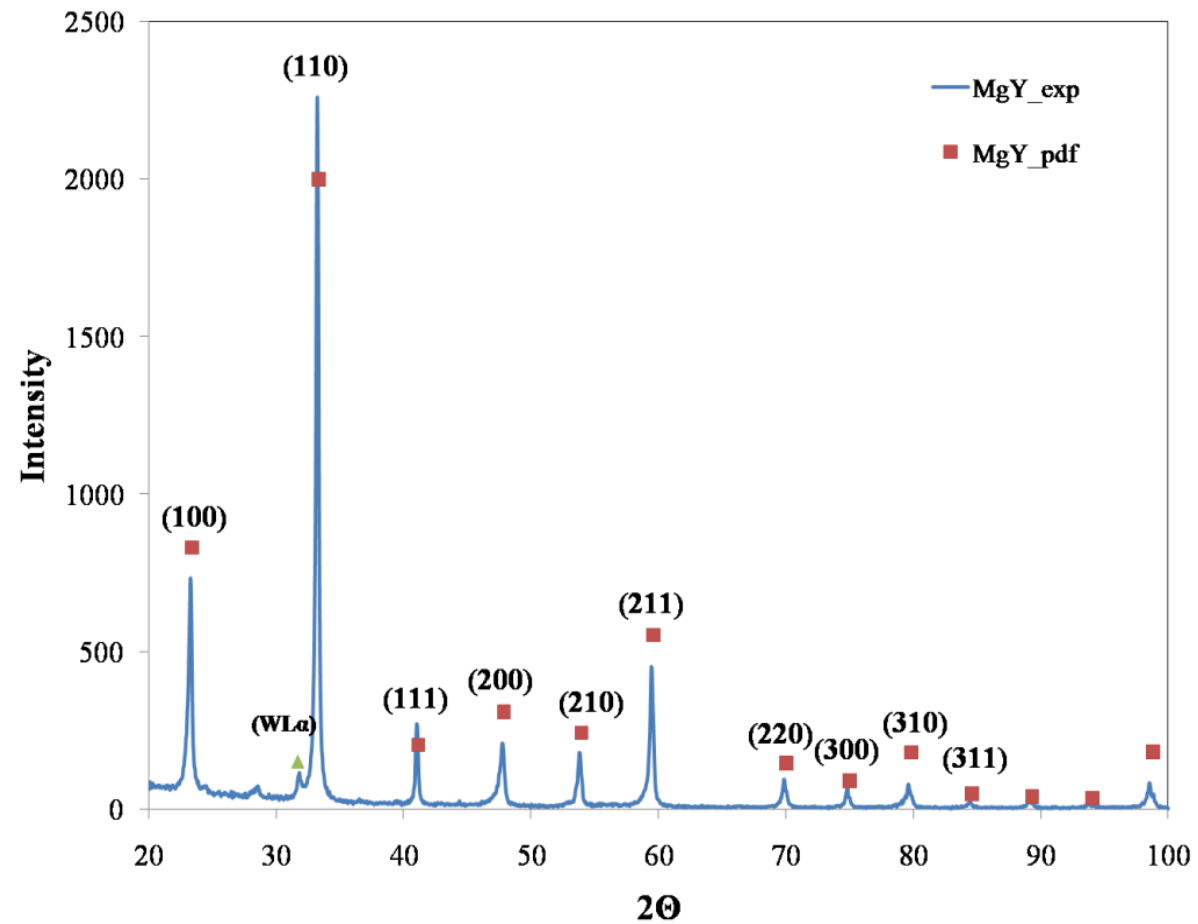

f)

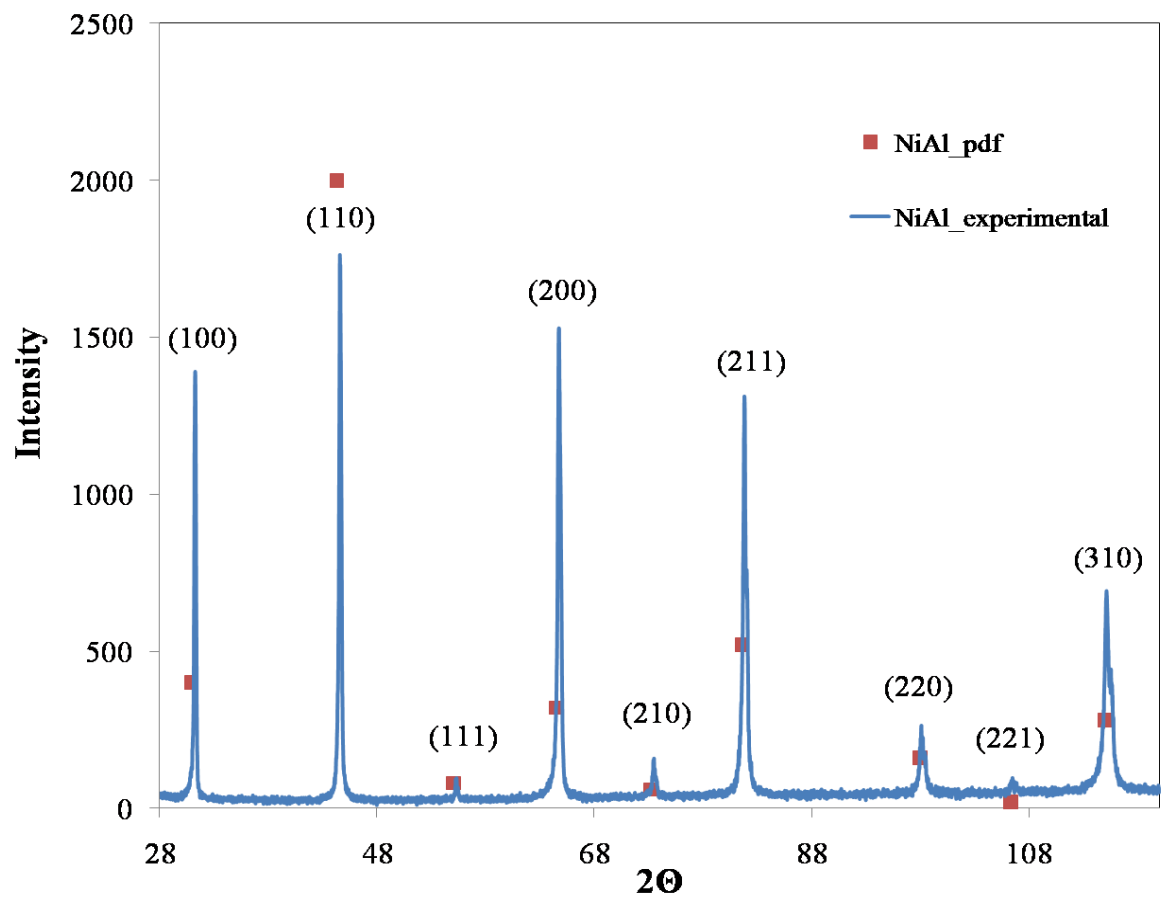

Figure 2.1: X-ray Diffraction patterns used in the phase analysis of a)AgY b)AgEr c) CuY d)CuDy e)MgY and f) NiAl 


\subsubsection{Chemical ordering}

All the X-ray diffraction patterns have superlattice reflections ((100), (111), (210), etc.), confirming the presence of an ordered B2 crystal structure (Fig.2.2). In the cases of $\mathrm{AgY}, \mathrm{MgY}$ and $\mathrm{MgCe}$ (Fig. $2.2 \mathrm{a}, \mathrm{b}, \mathrm{c}$ ), some additional oxide peaks appear in the scans of the powdered and annealed material, which were not present in the as-cast material. It appears that oxidation was not prevented, despite encapsulation within evacuated quartz ampoules, back-filling with Ar, and gettering with Ti. All of the alloy constituents $\mathrm{Mg}, \mathrm{Y}, \mathrm{Ce}, \mathrm{Ag}$, and $\mathrm{Y}$ have very high affinity for oxygen and strong oxidizing tendencies at high temperatures.

The integrated intensity values of (100) and (200) reflections were used to eliminate any possibility of texture effects, though only the weakest textures are likely in the powdered samples that were prepared. Rather than making multiple measurements of the order parameter to determine a standard deviation, an error analysis was performed using standard methods of error propagation [27]. This enabled incorporation of the uncertainty associated with measured intensities as well as the regression analysis carried out to determine temperature factor [28]. A sample plot for the determination of the temperature factor (Eqs. 5-7) is shown in Figure 2.3. The values of the order parameters obtained (and their calculated uncertainties) are presented in Table 2.3. All of the compounds are shown to be ordered. ${ }^{\text {iii }}$

iii In the case of $\mathrm{AgY}$, an $\mathrm{S}$ value greater than 1 was obtained from equation (8). Though it is physically impossible for the order parameter to be greater than one, such values can result because of experimental uncertainties [19,30], and they have been interpreted to mean the long range order is essentially perfect [31], within the limits of experimental error. 
Table 2.3: Measured order parameters

\begin{tabular}{|c|c|}
\hline Alloy & $\boldsymbol{S}$ \\
\hline $\mathrm{AgY}$ & $1.0 \pm 0.05^{\text {iii }}$ \\
\hline $\mathrm{MgCe}$ & $0.73 \pm 0.01$ \\
\hline $\mathrm{MgY}$ & $0.88 \pm 0.01$ \\
\hline $\mathrm{NiAl}$ & $0.95 \pm 0.02$ \\
\hline
\end{tabular}

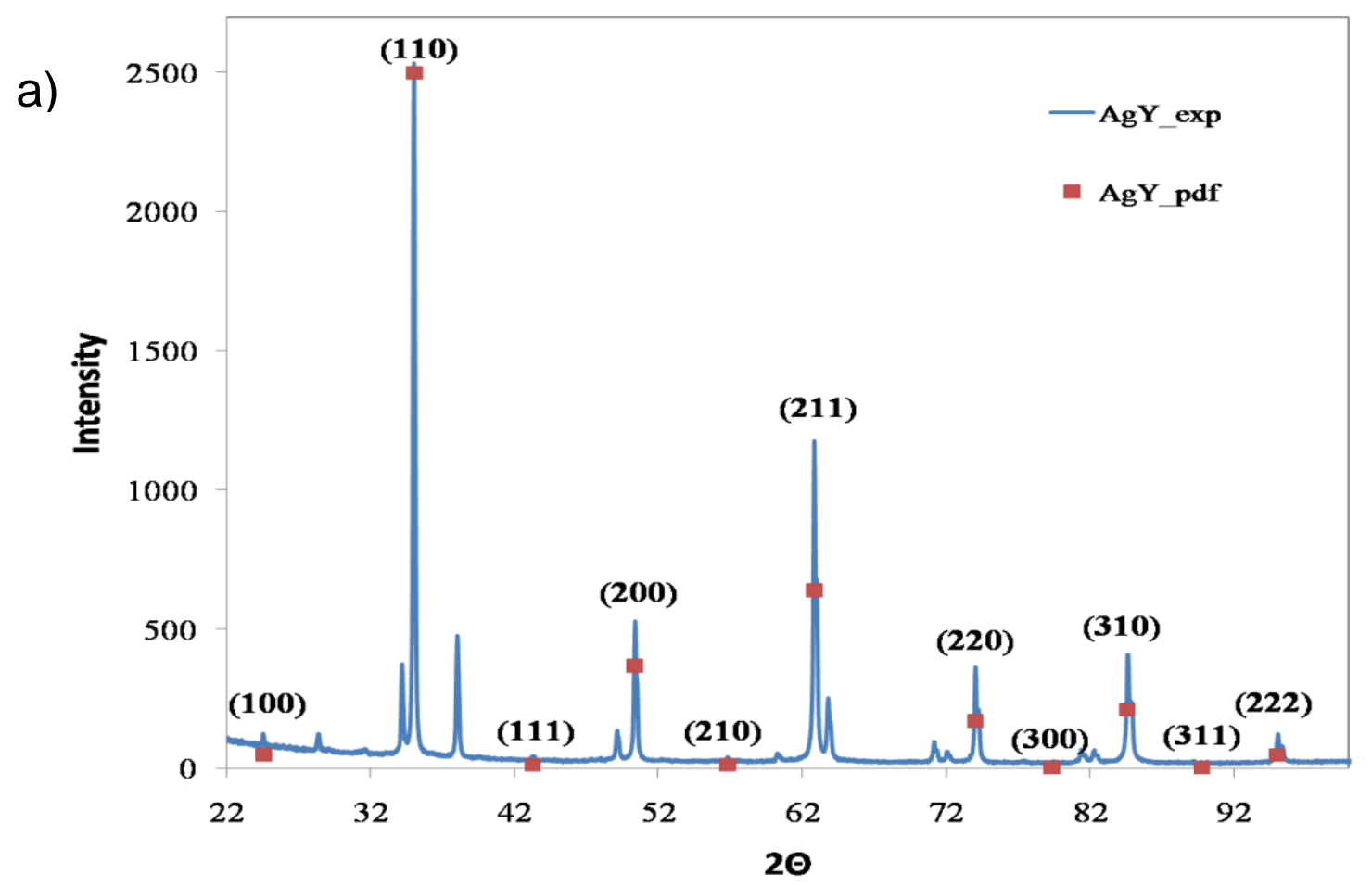


b)

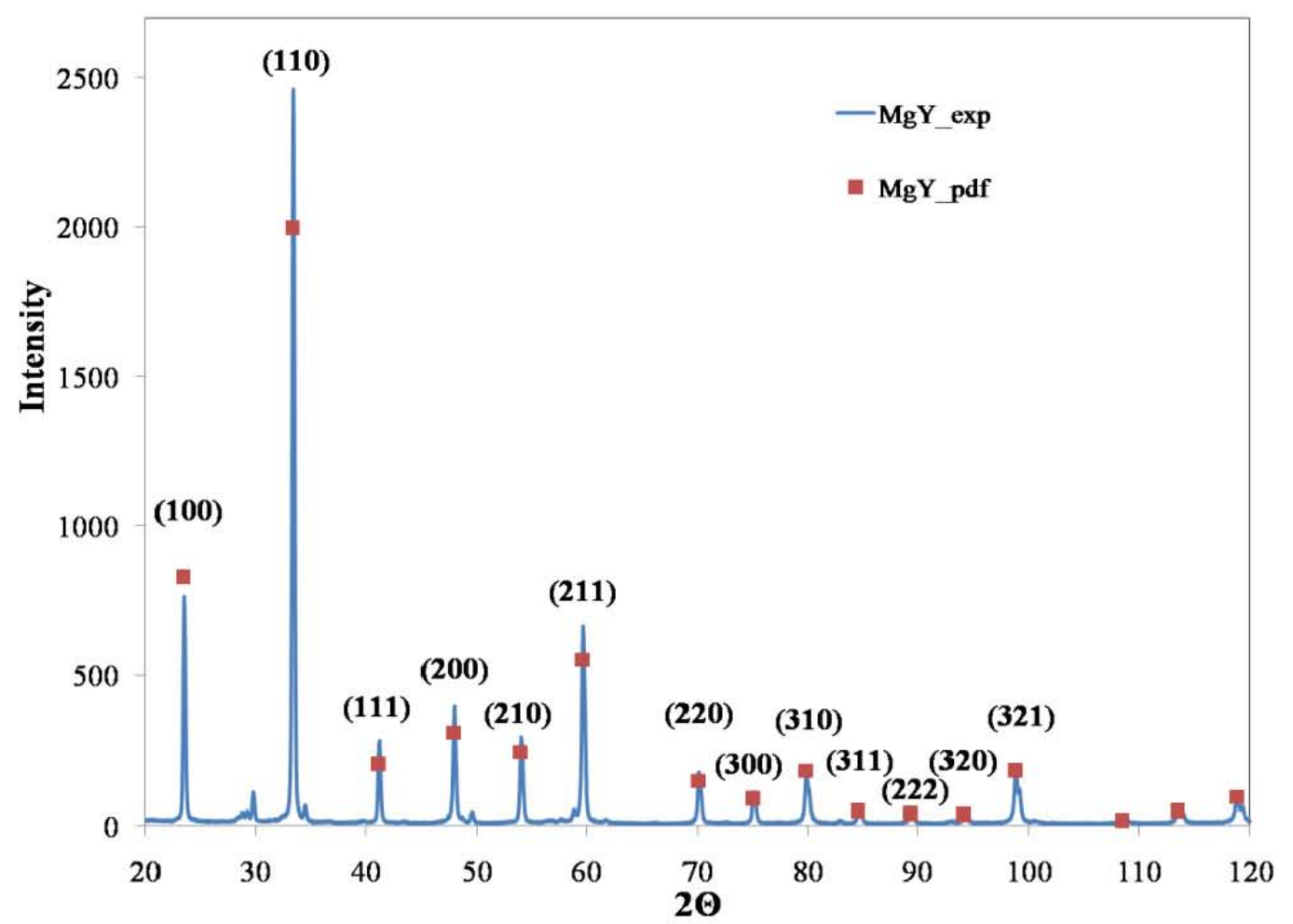

c)

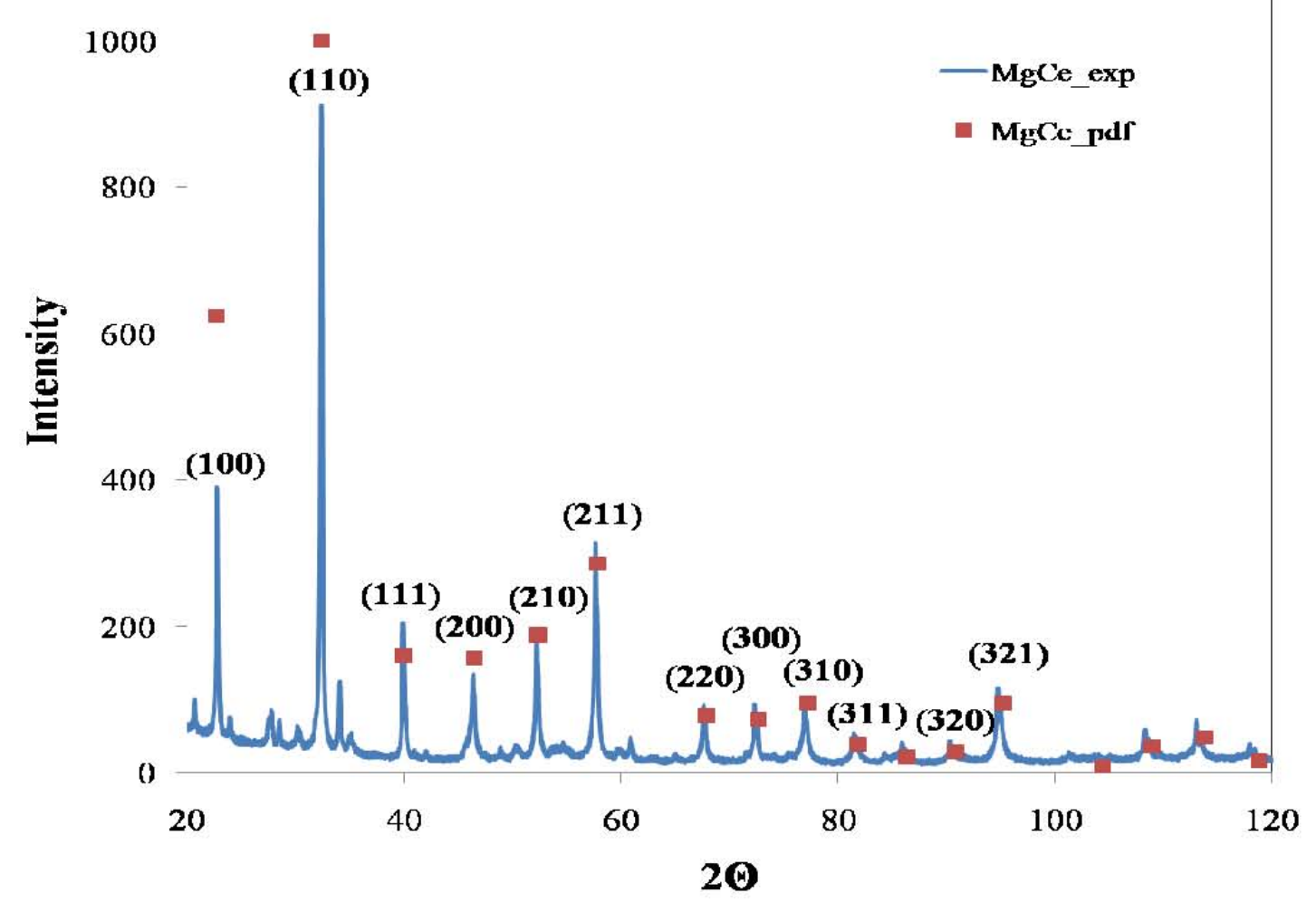




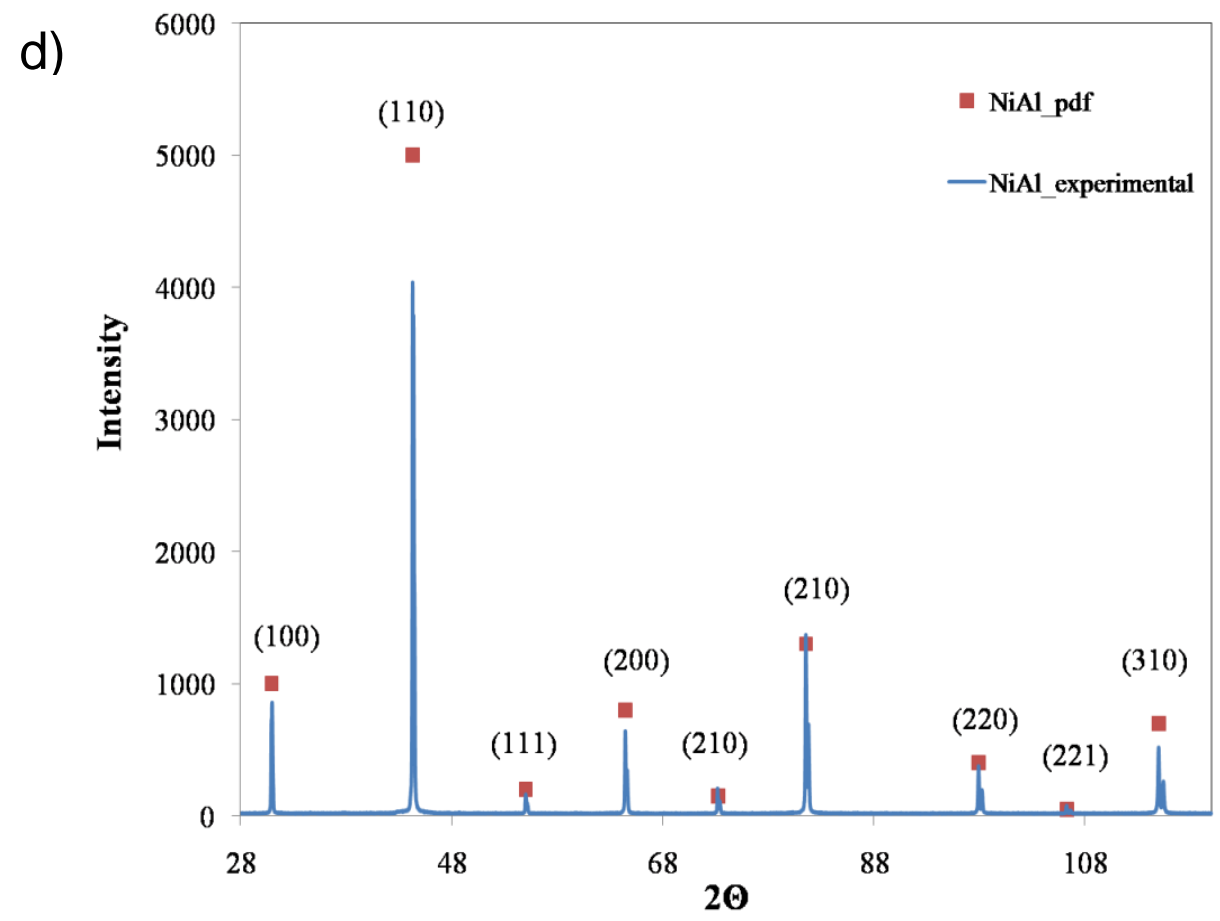

Figure 2.2: X-ray Diffraction patterns used in the order parameter determination of a)AgY b)MgY c)MgCe and d) NiAl

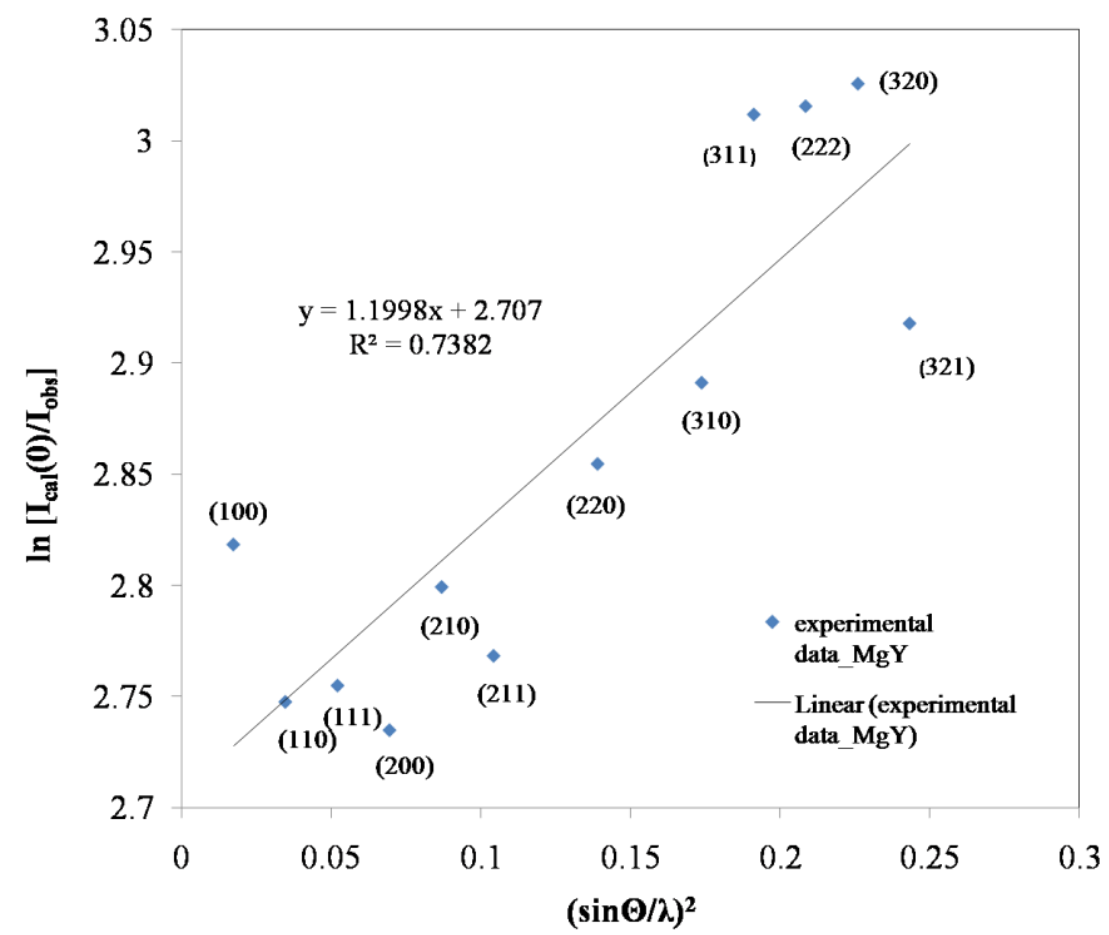

Figure 2.3: A typical plot of MgY used to obtain the temperature factor. 


\subsubsection{Crystallographic Texture}

The texture data are presented in equal area projection pole figures (Fig.2.4). The hot-rolled CuY (Fig.2.4 b) and AgEr (Fig.2.4c) are very weakly textured, while alloy AgY (Fig.2.4a) does exhibit a moderate texture strength, with peak intensities of 3.59 in the (100) pole figure. The cast MgY (Fig.2.4d) sample is shown to be randomly textured. It is important to note that none of the materials exhibit a particularly strong crystallographic texture, which could serve as a categorical explanation for why B2 compounds with $<100>$ "cube" slip as the primary deformation mechanism would be observed to be ductile. If a polycrystalline material is strongly textured, in the extreme case, every grain is oriented in the same direction and it is effectively a single crystal and hence does not need to satisfy von Mises criterion for ducility.
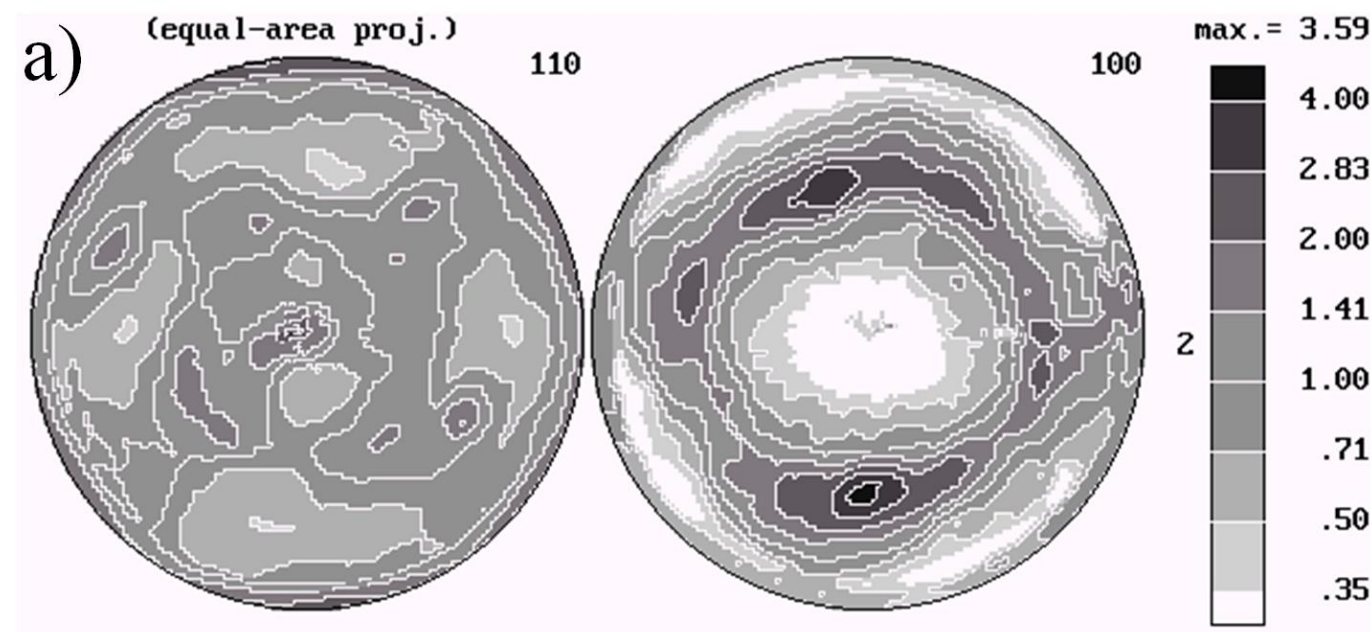

1

$\log$. scale 

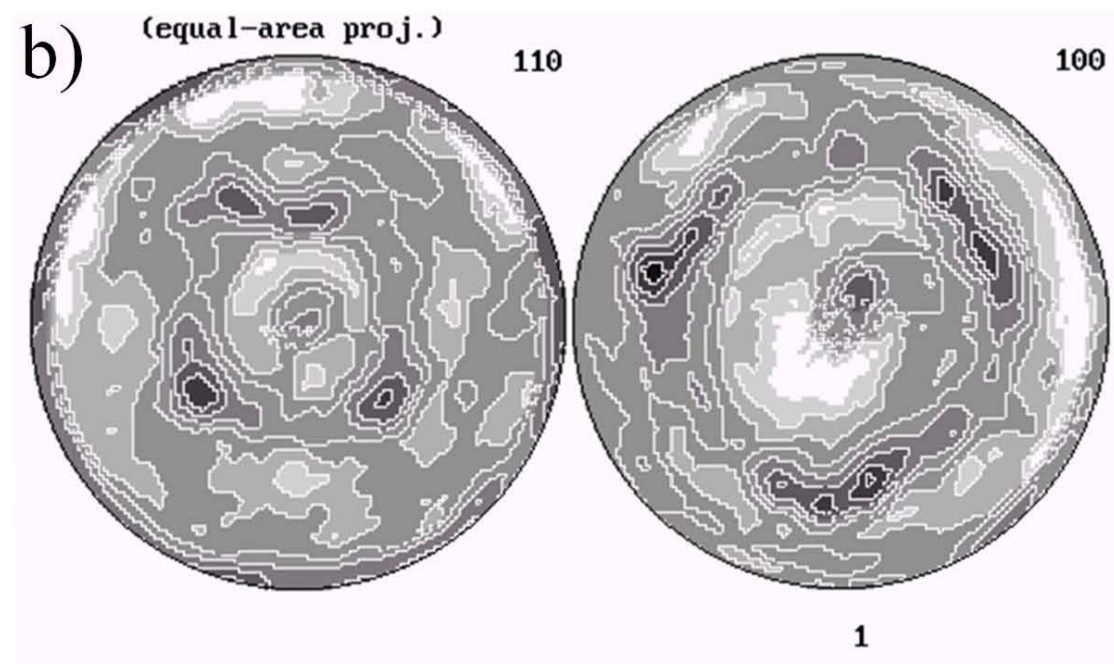

$\max .=3.63$

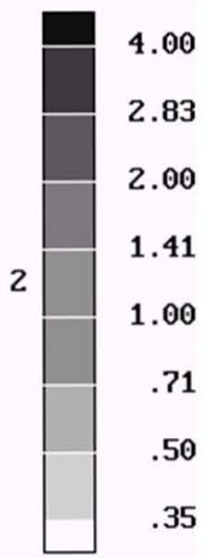

$\log$. scale
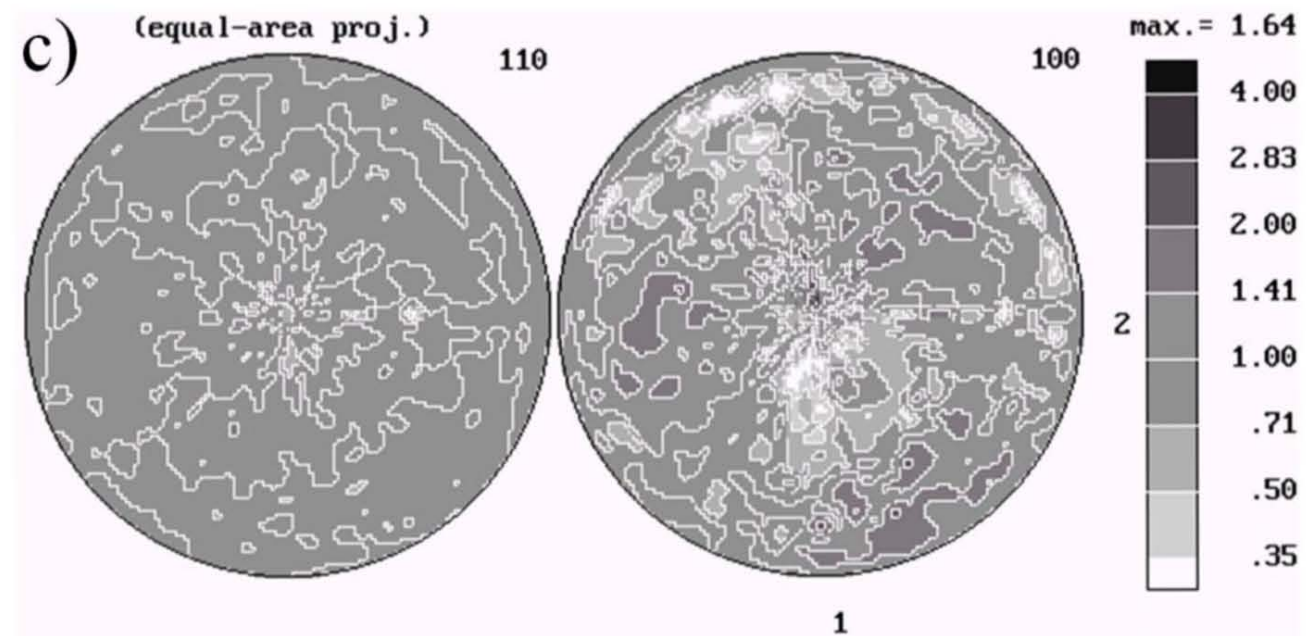

log. scale 

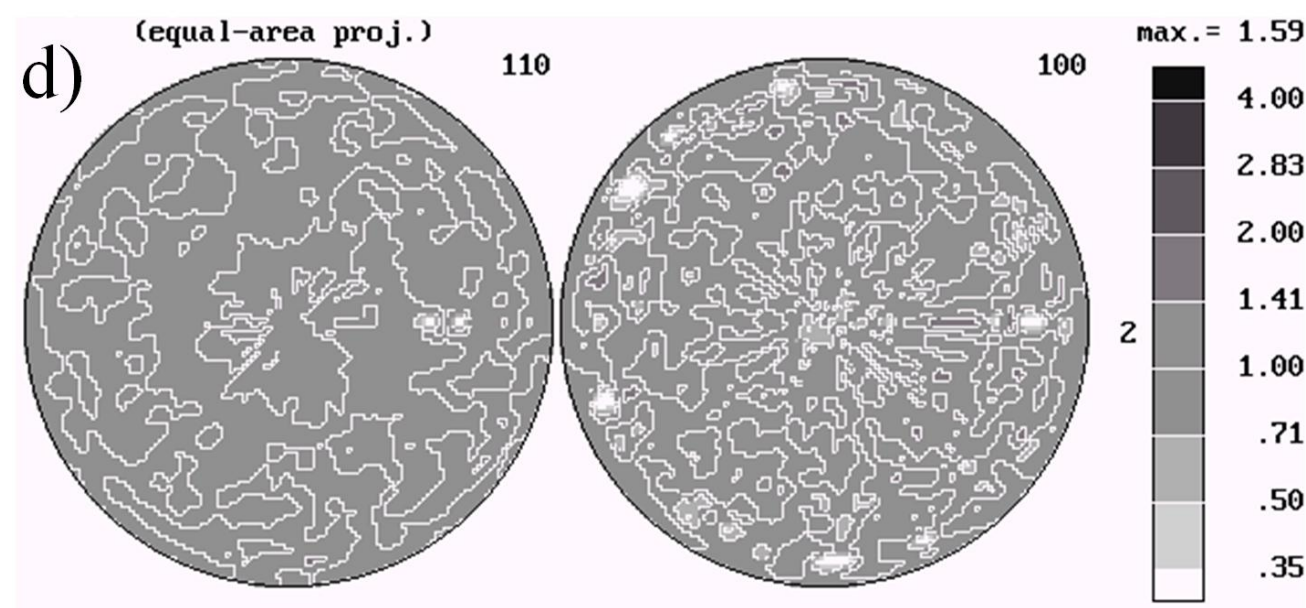

1

$\log$. scale

Figure 2.4: Pole figures representing the texture of rolled a) AgY b) AgEr and c) $\mathrm{CuY}$, with rolling direction vertical and transverse direction horizontal, and d) cast MgY, cylinder axis out of the page.

\subsection{Discussion}

\subsubsection{Phase Content}

The X-ray quantitative phase analysis (Fig.2.1 and Table 2.2) clearly shows that the ductile B2 materials are not single phase. They generally contain some amount of $\mathrm{M}_{2} \mathrm{R}$ as well as $\mathrm{R}_{2} \mathrm{O}_{3}$ phases. It is difficult to produce line compounds with perfect stoichiometry. For instance, it is possible that the vapor pressure of one constituent is higher than another, which would lead to an alteration of the composition of the melt during arc melting. In the present case, it is suggested that even when the overall metal alloy composition is stoichiometric, the strong affinity of rare-earth elements for oxygen reduces the effective rare-earth content. This explains why the departure from 

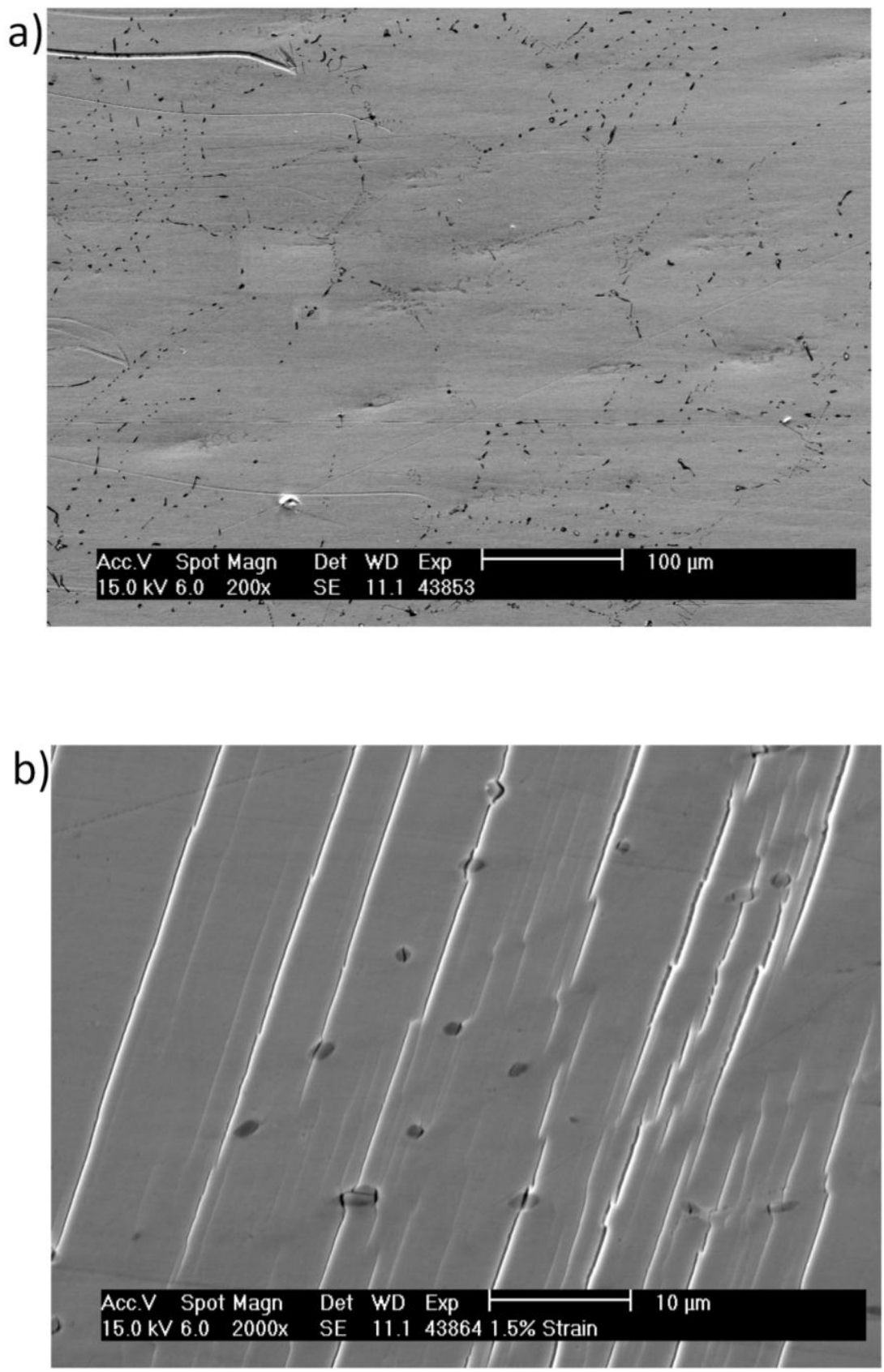

Figure 2.5: a) Micrograph of $\mathrm{AgY}$ showing second phases $\operatorname{Ag}_{2} \mathrm{Y}$ and $\mathrm{Y}_{2} \mathrm{O}_{3}$. b) Micrograph of AgY showing interaction between cracks and second phase particles. 
stoichiometry is always on the transition metal rich side, resulting in the formation of the $\mathrm{M}_{2} \mathrm{R}$ phase (see Fig. 1.2). AgY samples made at the respective laboratories Ames, ORNL (see Fig.2.5 for a microstructure overview), and UVa all contained similar phase mixtures.

Even intrinsically ductile materials can exhibit brittle behavior due to the presence of brittle second phases as seen in $\mathrm{B} 2 \mathrm{NiGa}[32,33]$. Tensile tests carried out on cast and annealed AgY samples clearly show an interaction between cracks and second phase particles (Fig.2. 5b). However, in spite of this fact, the samples still exhibited a ductility of 4-5\%. We find it significant that the MR compounds exhibit significant ductility $[1,9]$ in spite of the fact that they contain measureable quantities of second phase particles, which has not been previously reported.

\subsubsection{Chemical Ordering}

The order parameter study has shown that, like NiAl, AgY is fully ordered (Table 2.3). Traditionally, disorder and departure from stoichiometry have been shown to increase the ductility. This has been observed in B2 Fe-rich FeAl, where disorder makes slip of $<111>$ dislocations easier [34]. However, that does not seem to be the case in this family of materials; because despite the fact that $\mathrm{AgY}$ is fully ordered, it is ductile. Although we have not observed the Mg-based compounds to exhibit significant tensile ductility, it is still worth noting that both $\mathrm{MgY}$ and $\mathrm{MgCe}$ are experimentally observed to have high order parameters, though they are slightly lower than NiAl and AgY. This result validates theoretical predictions that such compounds will have a very strong tendency to order [35]. 


\subsubsection{Crystallographic Texture}

None of the materials studied are strongly textured (Fig. 2.4) Therefore, it cannot be argued that the anomalous ductility is due to a strong texture that alleviates the usual requirement for five independent slip systems [17]. In fact, as-cast and homogenized samples of $\mathrm{AgY}$ still exhibited $\sim 5 \%$ elongation at room temperature, despite having a presumably weak texture like the other cast samples examined in this study. CuY is one of the more ductile MR compounds observed to date, yet it is shown to be essentially randomly textured, even in the hot-rolled condition. Nevertheless, the moderate texture present in AgY may explain why it accommodates more strain before failure than the other ductile B2 intermetallics and why it is regarded as the most ductile B2-rare-earth intermetallic explored to date.

Evidence in support of this hypothesis is provided by extending the results of Wollmershauser, et al [36, 37], who have recently used a combined approach involving in-situ neutron diffraction and polycrystal plasticity modeling to determine the active deformation mechanisms within ductile B2 intermetallics. They began with a comparative study of archetypal ductile and brittle B2 compounds ( $\mathrm{CuZn}$ and $\mathrm{NiAl}$, respectively) and compared the results with $\mathrm{AgCe}$, a representative of the potentially ductile MR family of compounds. It was clearly shown that the initial deformation of $\mathrm{AgCe}$ is accommodated by $<100>\{011\}$ slip, but that another mechanism is required to explain the behavior at the highest stress levels. Further study of similarly ductile B2 compounds, CoTi and CoZr, has more clearly shown a transition during which strain accommodation by exclusively $<100>\{011\}$ slip changes to include either $<110>\{110\}$ or $\langle 111>\{110\}$ slip systems. The hardening rates are initially so steep that previous 
investigations of CoTi mistakenly identified the observed transition in plastic deformation mechanisms as the yield point. Thus, a secondary finding of these studies is an explanation of the very rapid initial strain hardening rates [38] that are common to all
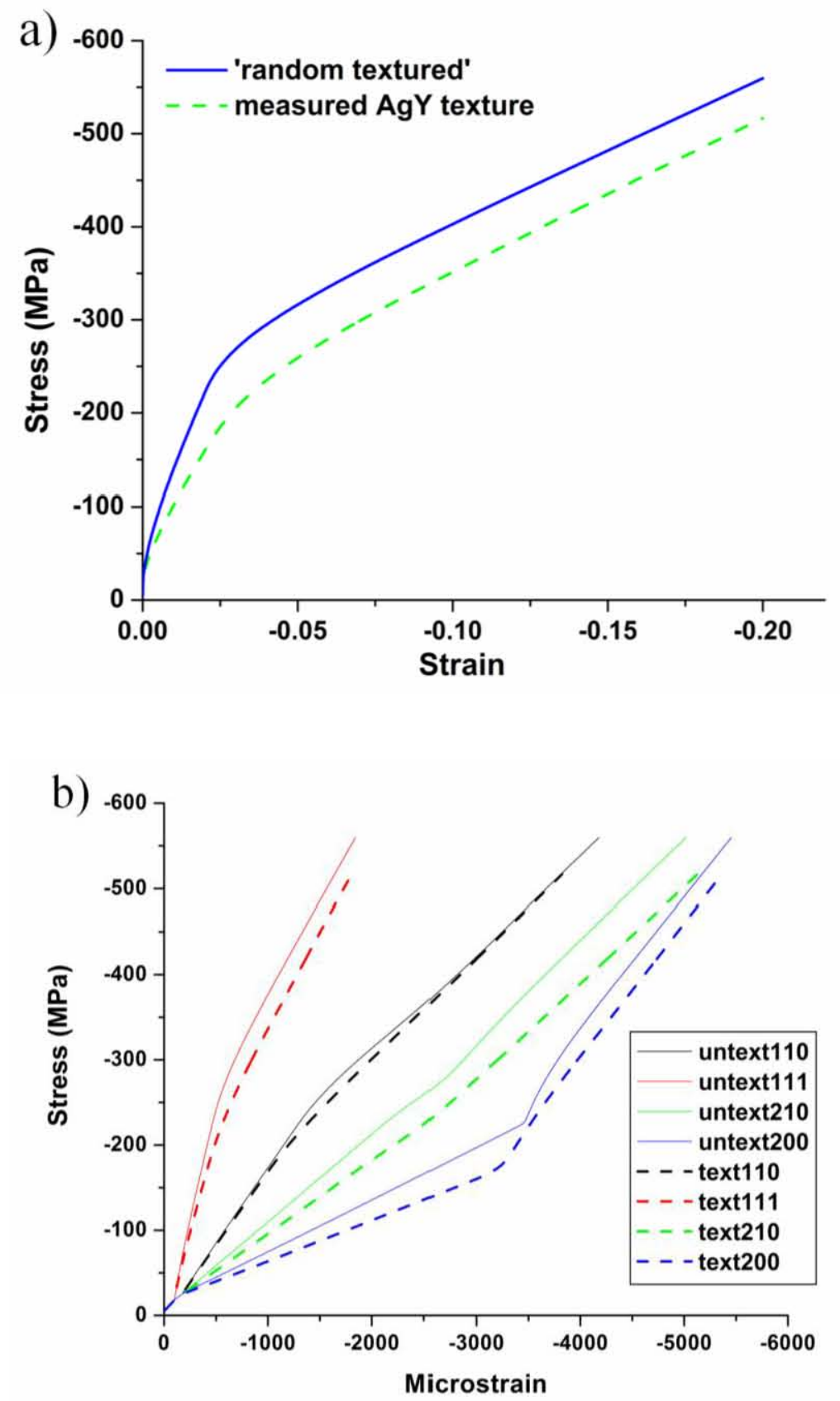

Figure 2.6: EPSC modeling of the mechanical behavior of AgY, a) Stress vs. Strain curve b) Internal strains, which show that the stresses built up are lower in the presence of a moderate texture. 
of these compounds in the small strain regime, where only $<100>\{011\}$ slip is observed. In short, the lack of independent slip systems offered by the primary slip mode causes great heterogeneity in the strain distribution between the grains, giving rise to a sort of composite effect as some grains remain largely elastic even well past the initial yield point.

It is not the purpose of the present paper to review the details of modeling performed previously. Rather, we simply present the effect that a moderate texture can have on the flow stress and internal stress development. For further information on EPSC modeling, the interested reader is guided to [39]. For the present exercise, we use slip parameter data, which is the best fit to the behavior of CoZr, and changed only the input textures. The measured texture for AgY was used to describe the orientation of the grains in Case 1, and a model material with no preferred orientation is used in Case 2. Compared to the untextured Case 2, it can be seen that the moderate, Case 1, texture of hot-rolled AgY allows for a reduction in the flow stress (Fig.2.6a). This has the important implication that even a moderate texture may serve to extend the ductility since the material would reach the failure stress at a higher value of strain. At any value of strain beyond the yield point, the sample with a moderate texture will have a lower elastic strain energy due to a lower stress. This is significant, since elastic strain energy is a primary driving force for fracture,

Figure $2.6 \mathrm{~b}$ presents predictions of the internal strains within differently oriented grains. The grain orientations have been parsed based upon how they would contribute to different diffraction peaks during an in-situ deformation experiment. This kind of presentation is useful for comparison with previously published in-situ neutron 
diffraction data. Note that strong inflections in the curves denote a transition in the strain carrying mechanism. The textured material undergoes the transition to secondary slip at a lower macroscopic stress, and this will ultimately have an even greater effect (on the delay in fracture) than the simple fact that the moderate texture provides a larger volume fraction of grains which are oriented such that primary slip can more easily accommodate the deformation prior to the transition. Again, although the presently highlighted role of texture does not provide an explanation for the general phenomenon of ductility in these materials, it does provide an explanation for why AgY has exhibited the greatest level of ductility to date.

Although the present study eliminates many possible reasons for the larger than expected ductility, the list is not exhausted. A recent first-principles modeling study [40] has suggested that the absence of $d$-band electrons near the Fermi level may be used to predict the ductility of B2 compounds, a concept which could be used in future ductile alloy development.

\subsubsection{CoZr and CoTi}

As stated in chapter 1 , out of the several anomalously ductile MR compounds, this dissertation focuses on CoTi and CoZr. In light of this, it is important to ensure that the conclusions drawn in this chapter, based largely upon examinations of rare earth containing alloys, are also applicable to CoTi and CoZr. Details regarding the alloy preparation can be found in chapters 3 and 5. Details regarding the neutron diffraction measurements upon which the texture analysis is based are also given in chapter 5 . Both of the alloys show a weak texture with the $<111>$ direction parallel to the extrusion axis (Fig. 2.7). 
a)

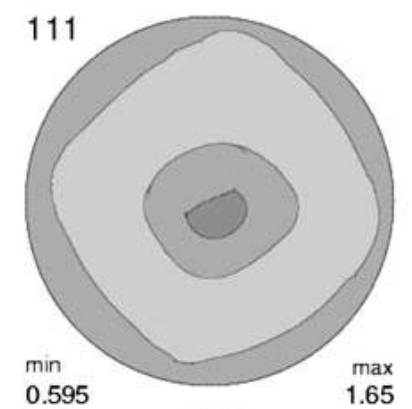

b)

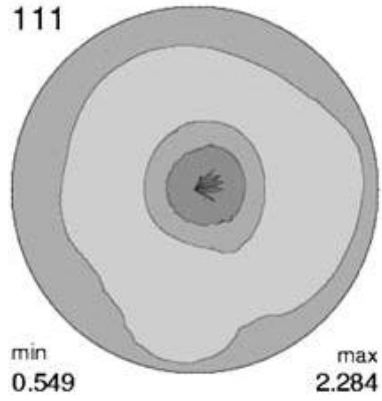

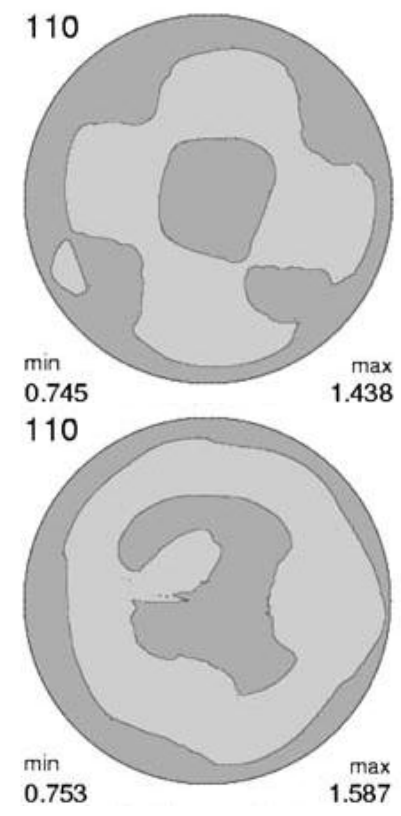

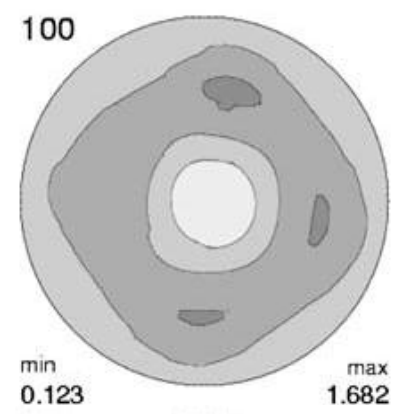

Figure 2.7: Pole figures of a) $\mathrm{CoZr}$ and b) CoTi with the extrusion axis out of the page. Both alloys have a weak texture with $<111>$ parallel to the extrusion axis.

Figure 2.8 shows $\mathrm{X}$-ray diffraction patterns obtained from $\mathrm{CoZr}$ and CoTi in the bulk form. Since both alloys contain Co, which has a K-absorption edge $(0.161 \mathrm{~nm})$ higher than the characteristic $\mathrm{Cu} \mathrm{Ka}$ radiation $(0.154 \mathrm{~nm})$, a higher background is observed. Nevertheless, by running slower scans (step size of $0.08^{\circ}$ and counting time per step of $40 \mathrm{~s}$ ) which allow for a larger number of counts, it is possible to observe some peaks with lower intensities. No evidence of secondary phases is present in the X-ray diffraction scans of either CoTi or CoZr (Fig. 2.8). This provides evidence that the presence of second phases is not needed for ductility. TEM and neutron diffraction experiments (chapters 3 and 5) carried out on both these alloys, as well as neutron diffraction experiments carried out by Wollmershauser, et al. [37] on the same heat of alloys, did not show the presence of any other phases which is in agreement with the $\mathrm{X}$ ray diffraction results. 
a)
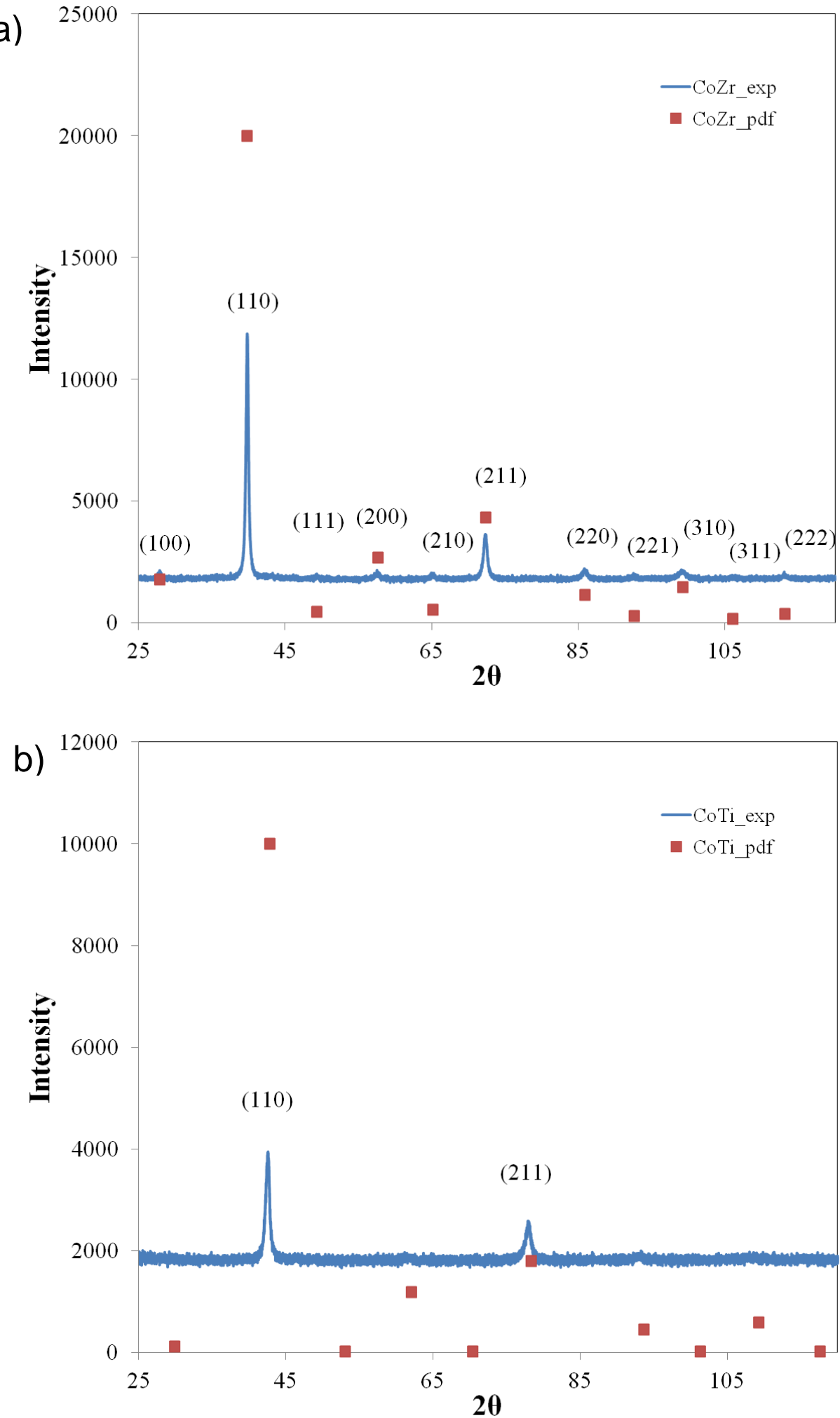

Figure 2.8: X-ray diffraction patterns of a) $\mathrm{CoZr}$ and b) CoTi. 
Figure 2.8 a shows the presence of superlattice reflections in CoZr. The order parameter was determined using equation 8 (neglecting the temperature factor) and was found to be 1 , indicating that CoZr is fully ordered. Again, neutron diffraction results (chapter 5) also show the presence of strong superlattice reflections. In the case of CoTi, because of the small difference in the scattering cross-sections of $\mathrm{Co}$ and $\mathrm{Ti}$, the intensities of the superlattice reflections were too low to be seen in figure $2.7 \mathrm{~b}$. However, superlattice reflections were clearly seen in diffraction patterns obtained using the TEM (Chapter 3), indicating that CoTi is indeed ordered, though a complete quantification of the order parameter was not conducted.

\subsection{Conclusions}

Measurable amounts of second phase impurities are observed in all the ductile B2 rare-earth containing compounds. This is not surprising, but it is important to note that the anomalous ductility observed in some MR compounds does not require a high level of phase purity, a fact which has not been previously reported. Secondly, the MR compounds are experimentally verified to be fully ordered and, therefore, do not require disorder or departure from stoichiometry in the primary phase to exhibit ductility, as has been observed for some other intermetallic compounds. Thirdly, because none of the materials examined have a pronounced texture, the possibility that texture is fundamentally responsible for the anomalous ductility is ruled out. However, the moderate texture present in AgY may explain the higher ductility in this material, as compared to other MR compounds, and polycrystal modeling confirms that the observed texture will lower the flow stress below that required in a randomly textured aggregate. 


\subsection{Acknowledgements}

The work carried out at the University of Virginia was sponsored by the National Science Foundation through a CAREER Grant DMR-0547981. Work in Oak Ridge National Laboratory was sponsored by the Division of Materials Sciences and Engineering, US

Department of Energy. We are grateful to Dr. Joachim Schneibel and Mr. Cecil A. Carmichael, Jr. (Oak Ridge National Laboratory) for providing the NiAl arc-casting.

\subsection{References}

1. Gschneiner K, et al. Nature Mater. 2003; 2:587-590

2. Mulay RP, Agnew SR, Scripta Mater. 2009; 61(11): 1036-1039.

3. Stumphy BD, Thesis (M.S.) 2006; Iowa State University

4. Rachinger WA, Cottrell AH, Acta. Metallurgica 1956; 4(2): 109-113.

5. Schulson EM, Teghtsoonian E, Philos. Mag. 1969; 19:155.

6. von Mises R, Z. Ang. Math. Mech. 1928;8,161-185

7. Saka H, et al. Phil. Mag.A, 1984;50: 65-70.

8. Potter DI, Materials Science and Engineering 1970; 5: 201

9. Russell AM, et al., Acta Metall. Mater. 2004; 52 :4033-4040.

10. Wu YR, Hu WY, et al. Physica B-Condensed Matter 2008; 403: 3792-3797.

11. Takasugi T, Masahashi N, Izumi I, Acta Metallurgica, 1987; 35: 381-391.

12. Russell AM, in Intermetallics Research Progress ,Nova Science Publishers: New York; 2008:213-235

13. Ishida K, Kainuma R, et al. Metall. Trans.A- 1991;22: 441-446. 
14. Baker I, Deformation \& Fracture of ordered intermetallics III ,TMS:

Cincinnati;1996:27-44

15. Wood DL, Westbrook JH, J.Nucl. Mater. 1964;12:208

16. Westbrook JH, Metallurgical Reviews. 1964;9:415

17. Webster D, Beryllium science and technology, vol. 1, New York: Plenum Press., 1979, p.133.

18. Hahn KH, Vedula K, Scripta Metall. 1989;23:7-12

19. Rozner AG, Wasilewski RJ, Journal of institute of metals 1966;94:169

20. Baker I, Schulson EM, Metall. Trans. A 1984; 15: 1129-1136.

21. Munroe Materials Preparation Center, Ames Laboratory US-DOE, Ames, IA, USA

22. Zhang Z, et al., Intermetallics 2005;13: 559-564

23. Cullity BD, 3rd Ed., in Elements of X-ray Diffraction, Pearson: 2001, pp. 347-361.

24. Warren BE, in X-Ray Diffraction, Addison Wesley: 1968, pp. 208-213.

25. Xiao H, Baker I, Acta Metall. Mater. 1993; 42:1535-1540.

26. Kallend JS, et al., Mater. Sci. Eng.A. 1991; 132: 1-11.

27. Taylor JR, An Introduction to error Analysis, Oxford University Press: 1981, Chapter

3, pp. 40-80.

28.. Ibid. Chapter 8, pp.153-172.

29. Hughes T, et al., Journal of Applied Physics 1971;42: 3705-3716

30. Keating DT, Warren BE, Journal of Applied Physics 1951;22: 286-290

31. Chipman D, Warren BE, Journal of Applied Physics 1950;21: 696

32. Seybolt AU, Westbrook JH, Acta Metall. 1964;12: 449-460 
33. Westbrook JH, Metall. Mater. Trans. A, 1977; 8A: 1327

34. Vedula K. FeAl and $\mathrm{Fe}_{3} \mathrm{Al}$, in: Westbrook JH, Fleischer RL (Eds.). Intermetallic Compounds: Principles and Practice. Vol.2. New York: John Wiley \& Sons, Ltd.; 1995. p. 199-209 [chapter 9].

35. Shang SL, Hector LG, et al. Journal of Physics-Condensed Matter 2009;21(24): 12.

36. Wollmershauser JA, Kabra S, Agnew SR, Acta Mater. 2009; 57 : 213-223.

37. Wollmershauser JA, Neil CJ, Agnew SR. Metall Trans A 2010; 41A(5): 1217-29.

38. Xie S, et al., Scripta Mater.2008; 58 :1066-1069.

39. Turner PA and Tomé CN, Acta Metall. Mater 1994; 42 : 4143-4153.

40. Gschneidner K, et al., Acta Mater. 2009; 57 : 5876-5881. 


\title{
3.Hard slip mechanisms in $\mathrm{B2}^{\mathrm{CoTi}}{ }^{i}$
}

\author{
R.P. Mulay and S.R. Agnew \\ Materials Science and Engineering, University of Virginia, \\ Charlottesville, VA 22904-4745
}

\subsection{Abstract}

Deformation mechanisms in B2 CoTi have been examined using $\mathbf{g} \cdot \mathbf{b}$ analysis in a transmission electron microscope (TEM) and in-grain misorientation axes (IGMA) obtained from electron backscattered diffraction (EBSD). The results from both techniques confirm that cube dislocations are activated at low stresses and that $<100\rangle$ $\{011\}$ and $<100>\{001\}$ are the dominant slip modes. However, in samples deformed beyond a transition in the strain hardening behavior, activation of a secondary deformation mechanism is confirmed. TEM analysis shows the presence of dislocations with $<110>$ and $<111>$ Burgers vectors and IGMA analysis indicates the activation of $<111>\{1 \overline{10}\}$ slip mode. The slip of dislocations with $\langle 110\rangle$ or $<111>$ Burgers vectors can reduce stress concentrations that could otherwise lead to premature fracture, thus providing a satisfying explanation for the anomalous ductility of CoTi and related compounds, like CoZr.

Keywords: dislocation, diffraction, intermetallic compounds, strain, ductility

\footnotetext{
${ }^{\mathrm{i}}$ R.P.Mulay and S.R. Agnew, Hard slip mechanisms in B2 intermetallic CoTi, Acta Materialia, 2012, accepted.
} 


\subsection{Introduction}

Many intermetallic compounds have attractive properties like high hardness, high melting point, good oxidation resistance, etc. However, their brittle nature has always been the limiting factor in their practical application [1]. B2 intermetallics with a high anti-phase boundary (APB) energy, like NiAl, are known to slip along the $<001>\{110\}$ slip mode [2-4] which provides only three independent slip systems and, hence, is unable to satisfy von Mises criterion for polycrystalline ductility, which requires 5 independent slip systems . Despite this, some B2 intermetallics like CoZr and CoTi [5-7], and more recently, $\mathrm{AgY}, \mathrm{CuY}$, etc., [8,9] have been shown to exhibit a significant amount of ductility. In particular, CoZr exhibited a tensile elongation of more than $20 \%$ prior to failure [10]. Transmission electron microscopy (TEM) studies and single crystal slip trace analyses carried out on $\mathrm{CoZr}$ and CoTi have unambiguously identified the presence of only $<100>$ dislocations [11-14].

Recent in-situ neutron diffraction and polycrystal plasticity modeling of CoTi and CoZr has exposed a transition in the strain hardening behavior in these alloys (Fig.3.1) and revealed that prior to this transition only the $\langle 100\rangle\{011\}$ slip mode is active, but past the transition a secondary mechanism is activated [7]. This prior work suggested that the hard slip mechanism is initially 23.5 or 30 times harder than the primary slip system, for $<111>\{1 \overline{10}\}$ and $<110>\{1 \overline{10}\}$ slip modes, respectively. However, this prior work was unable to discriminate which secondary mechanism was actually responsible. (Model predictions that made use of either mechanism reproduced experimental results equally well.) The present work uses $\mathbf{g} \cdot \mathbf{b}$ analysis in a TEM and in-grain misorientation axes 
(IGMA) obtained using EBSD to elucidate the deformation mechanisms active in CoTi, particularly past the transition in strain hardening.

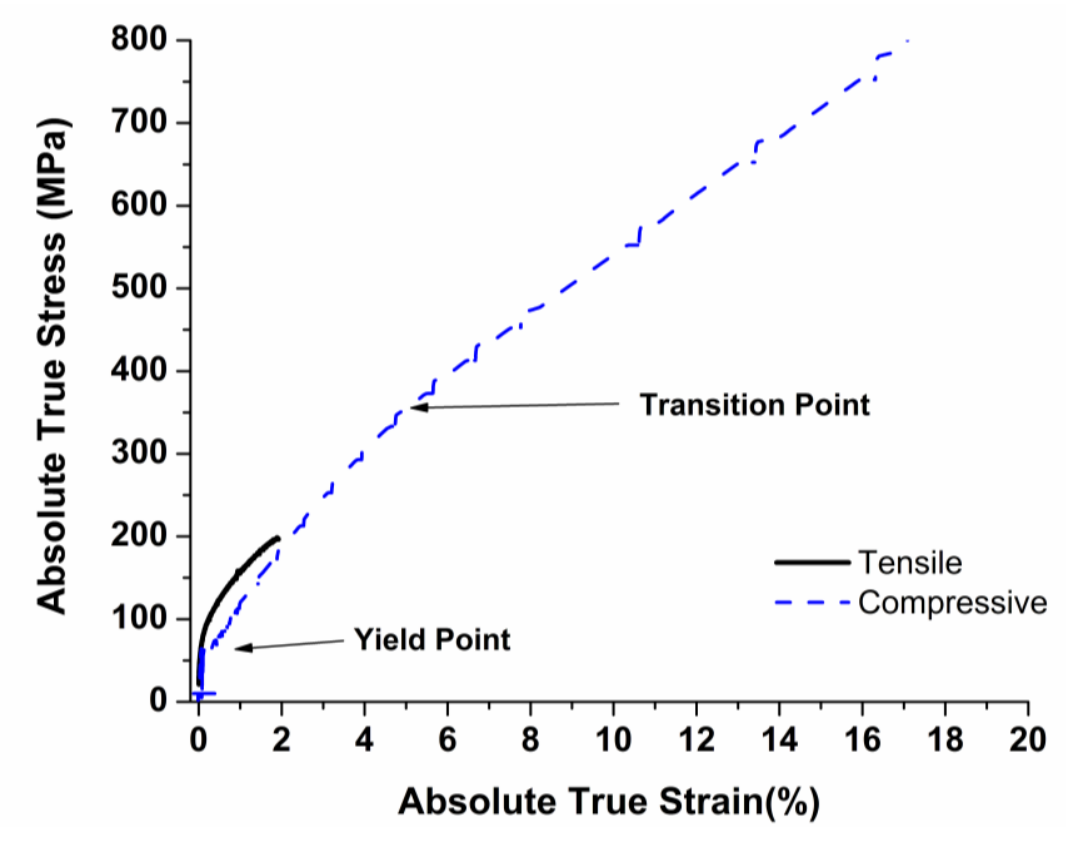

Figure 3.1: Compressive and tensile stress-strain curves of CoTi [7].

\subsection{Experimental procedure}

Cobalt (99.9 wt\%) and titanium (99.98 wt \%) purchased from Alfa Aesar were arc melted and drop cast as a cylinder (24.5 mm diameter) in an Ar atmosphere at the Oak Ridge National Laboratory (ORNL). The major impurities present in the raw materials are provided in Table 3.1. The mass loss was below $0.06 \%$. Hence, additional chemical analysis was not performed. The drop cast cylinder was homogenized in a horizontal tube vacuum furnace for 24 hours at $1000{ }^{\circ} \mathrm{C}$ and a pressure of $\sim 10^{-3} \mathrm{~Pa}$. The cylinder was then sealed in an evacuated stainless steel container and preheated to $1000^{\circ} \mathrm{C}$ for 1 hour prior to extrusion. Extrusion was performed at ORNL with a 4:1 reduction ratio, resulting 
in a final sample diameter of $\sim 12.7 \mathrm{~mm}$. The extruded billet was then turned on a lathe to remove the cladding and expose the alloy. Cylindrical compression specimens of

Table 3.1: Major impurities in raw materials.

\begin{tabular}{|l|c|c|c|c|c|c|c|c|c|c|c|}
\cline { 2 - 11 } \multicolumn{1}{c|}{} & \multicolumn{7}{|c|}{ Impurities (ppm) } \\
\hline Element & Al & C & Cr & Fe & Hf & N & Nb & Ni & O & Ta & W \\
\hline Cobalt & $<4$ & 6 & $*$ & $<3$ & $*$ & $<1$ & $*$ & 148 & 18 & $*$ & $*$ \\
\hline Titanium & 0.9 & 30 & 0.4 & 80 & $<0.01$ & 18.5 & $<0.2$ & 0.6 & 346 & $<5$ & 0.03 \\
\hline
\end{tabular}

* Not reported

diameter $4 \mathrm{~mm}$ and length $8 \mathrm{~mm}$, diameter $10 \mathrm{~mm}$ and length $20 \mathrm{~mm}$, rectangular compression specimens $6.5 \times 3.0 \times 3.0 \mathrm{~mm}$ and tensile specimens of gauge length 15.75 mm (Fig.3.2) were then cut with the loading axis along the extrusion direction using electro-discharge machining (EDM). Finally, the specimens were individually encapsulated in a quartz tube back filled with $\mathrm{Ar}$, and recrystallized at $1000{ }^{\circ} \mathrm{C}$ for 10 hours, with titanium as a getter, followed by furnace cooling as per previous studies in the literature [15]. For measuring the grain size, the recrystallized sample was mechanically polished up to 1200 grit silicon carbide paper and then with a $3 \mu \mathrm{m}$ diamond polishing compound. It was etched using a solution of $2.5 \mathrm{ml} \mathrm{HF}, 2.5 \mathrm{ml} \mathrm{HNO}_{3}$ and $95 \mathrm{ml}$ water [16]. 


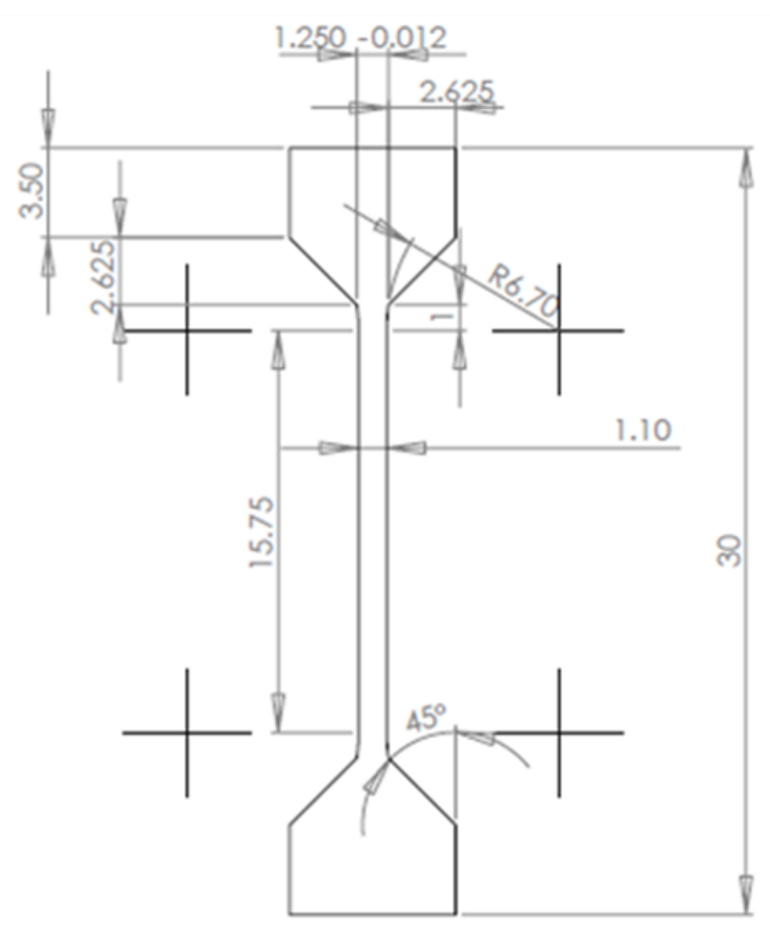

All dimensions are in $\mathrm{mm}$

Figure 3.2: Tensile specimen geometry.

Compression tests were carried out on an MTS screw-driven machine at a constant cross-head speed with an initial strain rate of $5 \times 10^{-4} \mathrm{~s}^{-1}$. A compression "cage fixture" was used in which the samples are compressed while the machine moves in a tensile mode [17]. This helped in achieving proper alignment in the load-train. Strain was calculated from the cross-head displacement. The strain values were also confirmed by measuring the strain directly off the sample after deformation. The large cylindrical samples (diameter $10 \mathrm{~mm}$ and length $20 \mathrm{~mm}$ ) were tested on a custom built Instron (Norwood, MA) load frame in the flight path of the time-of-flight neutron beam line, Spectrometer for Materials Research at Temperature and Stress (SMARTS), at the Manuel Lujan Jr. Neutron Scattering Center, (LANSCE, Los Alamos, NM). An 
extensometer was attached to the sample with rubber bands to measure the strain. The stress-strain curve is shown in Figure 3.1.Tensile tests were carried out on an Instron model 1321 (Norwood, MA) at a constant strain rate of $10^{-4} \mathrm{~s}^{-1}$ with an extensometer attached to the gauge length with rubber bands.

For TEM, samples deformed in compression up to 7 and $9 \%$ strains were cut into slices of $0.5 \mathrm{~mm}$ thickness, perpendicular to the loading axis, using electro discharge machining (EDM). This is a larger strain level than is normally employed in TEM studies, but as mentioned in the introduction (section 3.2), the interest here is in characterizing a deformation mechanism that only becomes active at higher strain levels. The slices were further ground down to a thickness of $\sim 0.1 \mathrm{~mm}$. $3 \mathrm{~mm}$ discs were then punched out of the slices and electropolished to perforation in a solution of $20 \%$ sulphuric acid and $80 \%$ methanol at a temperature of $-10^{\circ} \mathrm{C}$ and a voltage of $10 \mathrm{~V}$ using a Fischione Model 120 twin-jet electropolisher. TEM studies were carried out on a JEOL 2000FX operating at $200 \mathrm{kV}$. Burgers vectors of the dislocations were determined using the $\mathbf{g} \cdot \mathbf{b}$ method (e.g., [18]).

EBSD samples were prepared from deformed tension (2\% strain) and compression samples (16\% strain) by grinding with 600 grit silicon carbide paper followed by electropolishing in a solution of $20 \%$ sulphuric acid and $80 \%$ methanol [19]. No difference in the dislocation types between tension and compression samples is expected, rather the dislocation types active are thought to be dependent on the level of strain (and stress).The EBSD system is installed within a JEOL 840 scanning electron microscope and consists of a Nordlys NL-1 high-speed CCD camera and HKL Channel 5.0 software. Automatic beam scan mapping was carried out at an accelerating voltage of $30 \mathrm{kV}$, probe 
current of 0.1 to $0.6 \mathrm{nA}$, magnification of $500-750 \mathrm{X}$, working distance of $21 \mathrm{~mm}$ and a step size of $0.5 \mu \mathrm{m}$.

The deformation mechanisms in CoTi were analyzed by considering the dominant misorientation axis within a grain. This approach, called in-grain misorientation axis (IGMA), is based on slip induced lattice rotation about an axis perpendicular to the Burger's vector and slip plane normal and the determination of this rotation axis [20-24]. For a given slip mode, the IGMA is given by,

\section{IGMA $=\mathbf{b} \times \mathbf{n}$}

where, $\mathbf{b}$ is the Burger's vector and $\mathbf{n}$ is the slip plane normal. The IGMA for different slip modes in B2 alloys is shown in Table 3.2. Previous studies on $\mathrm{Zr}$ [20] and Be [21] have used Laue X-ray diffraction to determine the IGMA and gain insight into the active deformation modes. Recently, the deformation modes in Mg alloys [22, 23] and Ti [24] have been analyzed with IGMA obtained using EBSD. With EBSD it is possible to determine the IGMA in a large number of grains. It is to be noted that in IGMA analysis, we are only considering the geometrically necessary dislocation (GND) content and the IGMA is insensitive to the character of the dislocation [23].

Table 3.2: In grain misorientation axis (IGMA) corresponding to the different slip modes.

\begin{tabular}{|c|c|}
\hline Slip system & IGMA \\
\hline$<001>\{110\}$ & $<110>-6$ variants \\
\hline $\begin{array}{c}<001>\{100\} \\
\text { or } \\
<110>\{1 \overline{10}\}\end{array}$ & $<010>-3$ variants \\
\hline$<111>\{110\}$ & $<11 \overline{2}>-12$ variants \\
\hline
\end{tabular}


In the present work, the misorientation axis between neighboring scanning grid point pairs, with a misorientation of $0.5-2^{\circ}$, is plotted on an irreducible triangular projection (equal area projection) and presented with an intensity (with multiples of uniform distribution (mud)) contour plot by summing Gaussian distributions, with a half width of $10^{\circ}$, on every measured point. Qualitative distribution of the dominant IGMA was not found to be sensitive to the step size employed in acquiring the data. Since we are analyzing the misorientation axis within a grain, most of the data are at low misorienation angles $\left(\leq 2^{\circ}\right)$. However, the accuracy of IGMA determination is reduced at these low angles. In this study, we are interested in the dominant IGMA within a grain and not in the accuracy of IGMA at each point.

\subsection{Results}

\subsubsection{Optical metallography and mechanical tests}

The microstructure of $\mathrm{CoTi}$ after recrystallization at $1000^{\circ} \mathrm{C}$ for 10 hours is shown in Figure 3.3. The grain size was calculated using the linear intercept method and found to be approximately $71 \mu \mathrm{m}$. The tensile and compressive stress-strain curves for CoTi deformed at room temperature are shown in Figure 3.1. In compression, CoTi yields at around $-60 \mathrm{MPa}$ after which it strain hardens rapidly. Since the initial strain hardening is almost linear, a transition point in the strain hardening behavior can be defined using a $1 \%$ plastic strain offset similar to a yield point. While defining the transition point corresponding to the $1 \%$ plastic strain offset, the slope of the initial linear strain hardening is used akin to using the elastic modulus while defining the yield point. This transition in the strain hardening behavior is near -350 MPa after which the material strain hardens at a lower rate. In tension, it yields at a value of $100 \mathrm{MPa}$ but fractures at 
$\sim 200 \mathrm{MPa}$ and 2\% strain. Hence, this tensile tested sample did not strain enough to reach the transition in strain hardening behavior observed during compressive deformation. Notably, a similar alloy, CoZr, has exhibited $\sim 10 \%$ tensile elongation [7], which was enough tensile ductility to observe the transition in strain hardening rate. Although the yield and transition stresses in CoTi and $\mathrm{CoZr}$ are different, it is hypothesized that the two essentially deform in the same fashion, given the many similarities in their constitutive response $[7,25]$.

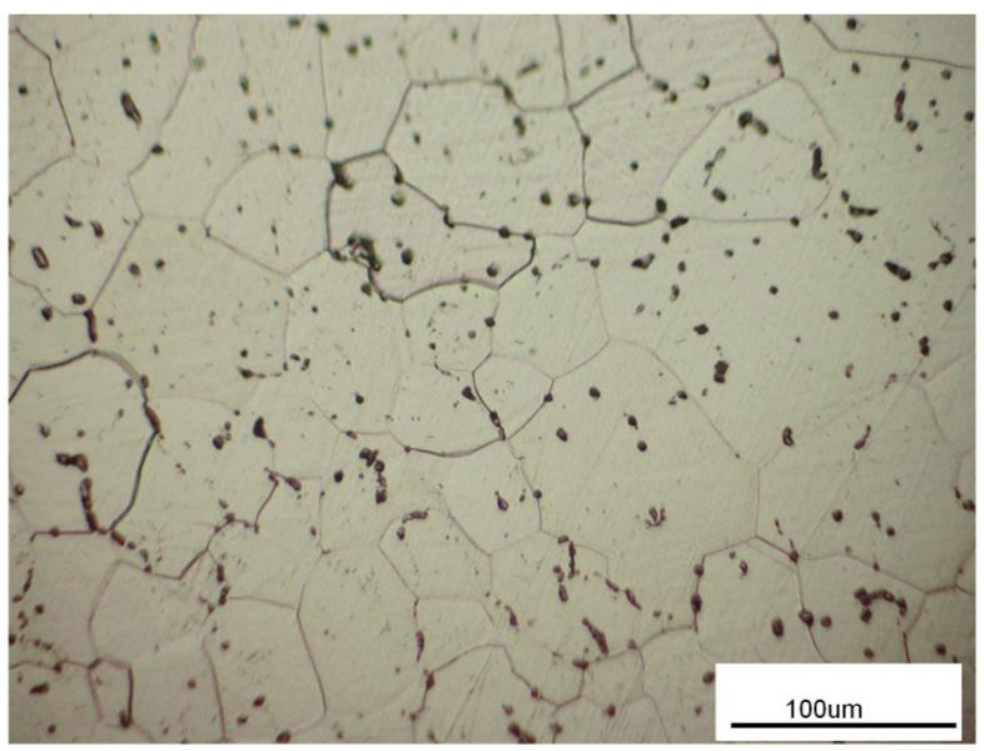

Figure 3.3: Optical micrograph showing the microstructure in recrystallized CoTi.

The black spots are etch pits. 


\subsubsection{TEM analysis of dislocation structures}
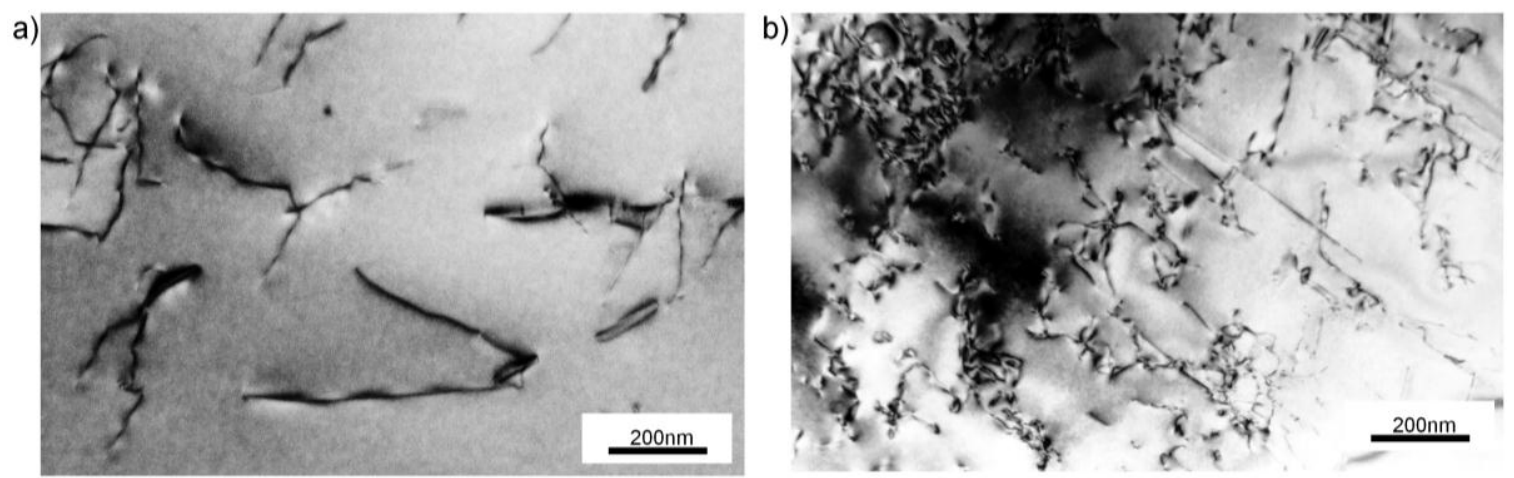

Figure 3.4: Bright field images of CoTi at different strain levels, a) recrystallized and b) $9 \%$ strain (beyond transition).

Bright field images of the microstructure in the recrystallized state and deformed up to $9 \%$ strain are shown in Figure $3.4 \mathrm{a}$ and $\mathrm{b}$, respectively. It can be seen that the dislocation density is much higher in the latter, as would be expected. This makes it challenging to isolate individual dislocations [26]. In order to alleviate this problem $\mathbf{g} \cdot \mathbf{b}$ analysis is usually carried out on samples strained only to a few percent or less. Because the dislocation types of interest in this study are only active at higher strains, TEM analysis was done on samples strained to $~ 7 \%$ (just beyond the transition) and $9 \%$ strain. 
a)

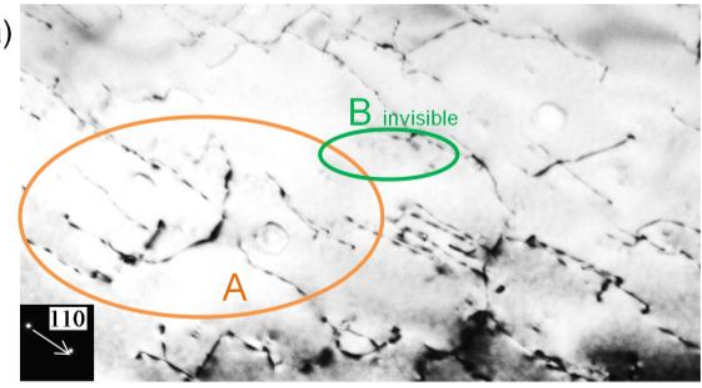

c)

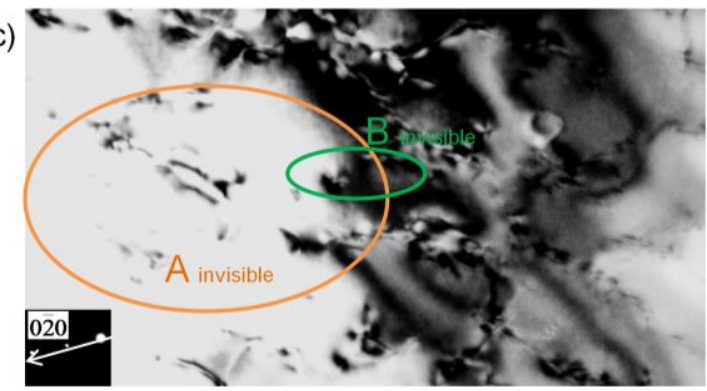

b)

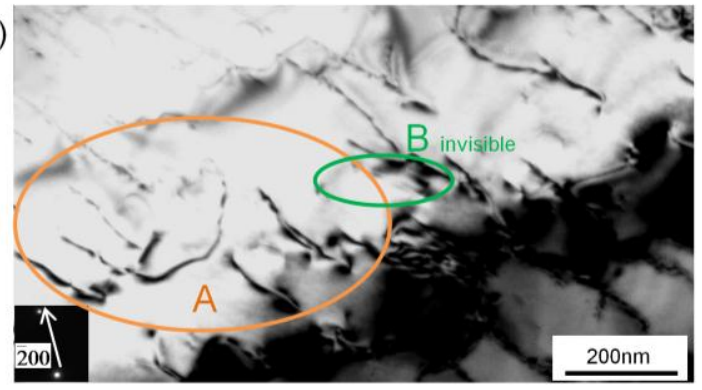

d)

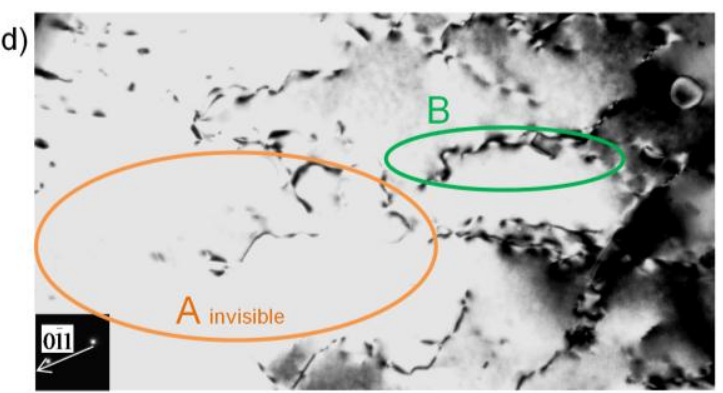

Figure 3.5: Series of two-beam bright field images of dislocations in CoTi deformed to $7 \%$ strain. The electron beam direction is near [001] for images $a, b$, $c$ and near [111] for image d. Analysis is shown in Table 3.3.

Table 3.3: Visibility-invisibility analysis of the dislocations in Figure 3.5.

\begin{tabular}{|c|c|c|c|c|}
\hline $\mathbf{b} \backslash \mathbf{g}$ & 110 & $\overline{2} 00$ & $0 \overline{2} 0$ & 011 \\
\hline A [100] & visible & visible & invisible & invisible \\
\hline B [001] & invisible & invisible & invisible & visible \\
\hline
\end{tabular}


a)

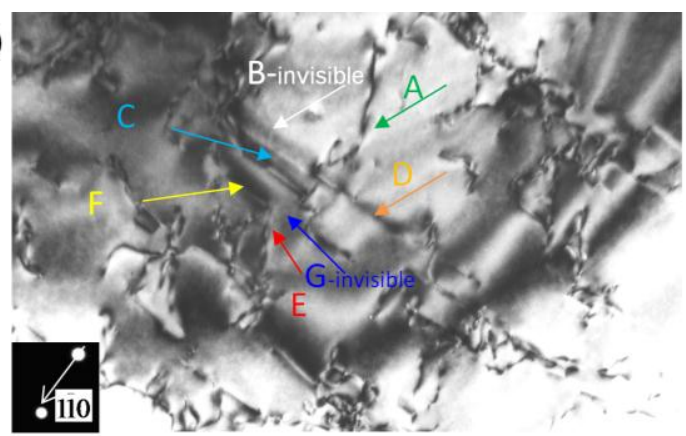

c)

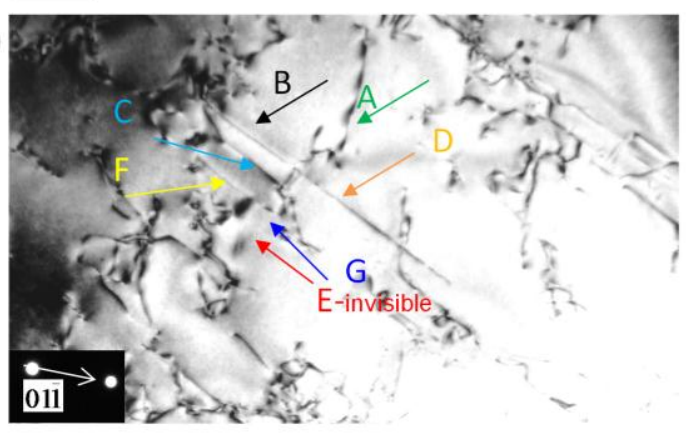

e)

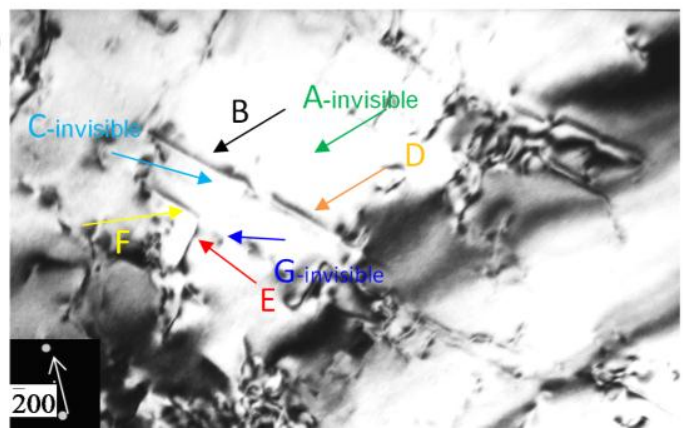

b)

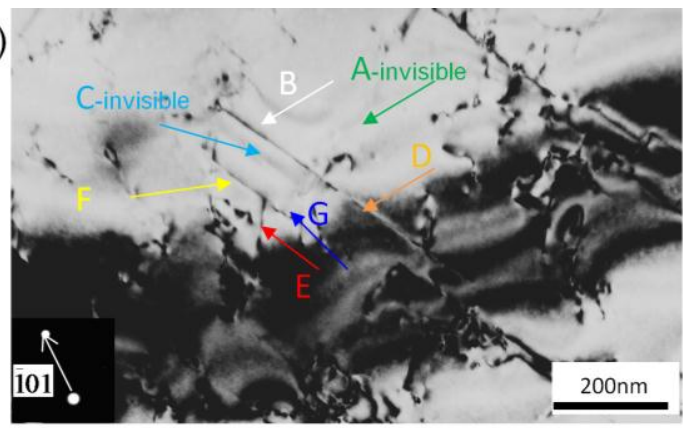

d)

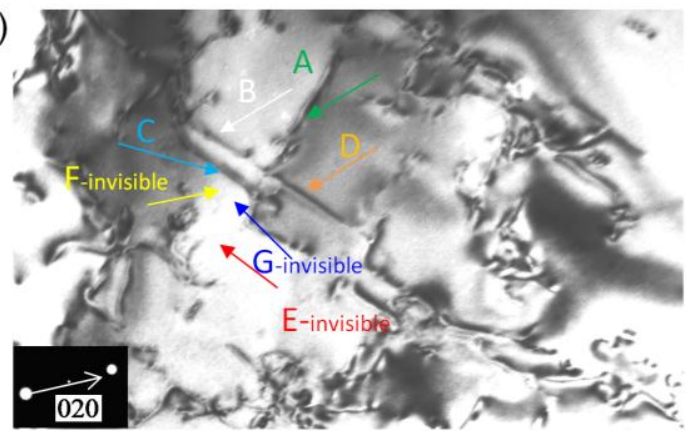

Figure 3.6: Series of two-beam bright field images of dislocations in CoTi deformed to $9 \%$ strain. The electron beam direction is near [111] for images $a, b$, $c$ and near [001] for images d and e. Analysis is shown in Table 3.4.

Table 3.4: Visibility-invisibility analysis of the dislocations in Figure 3.6

\begin{tabular}{|c|c|c|c|c|c|}
\hline $\mathbf{b} \backslash \mathbf{g}$ & $1 \overline{110}$ & $\overline{101}$ & 011 & 020 & $\overline{200}$ \\
\hline A [010] & visible & invisible & visible & visible & invisible \\
\hline $\mathrm{B}[110 / 111]$ & invisible & visible & visible & visible & visible \\
\hline $\mathrm{C}[010]$ & visible & invisible & visible & visible & invisible \\
\hline $\mathrm{D}[\overline{110}]$ & visible & visible & visible & visible & visible \\
\hline $\mathrm{E}[\overline{100]}$ & visible & visible & ple & ible & visible \\
\hline $\mathrm{F}[\overline{1} 01]$ & visible & visible & visible & invisible & visible \\
\hline $\mathrm{G}[001]$ & invisible & visible & visible & invisible & invisible \\
\hline
\end{tabular}


Figure 3.5 shows a series of two-beam bright field images of the sample deformed up to $7 \%$ strain. $\mathbf{g} \cdot \mathbf{b}$ analysis was carried out to determine the active Burgers vector(s) of the imaged dislocations. Dislocations in group A were found to have a Burgers vector of [100] while those in group B were found to have a Burgers vector of [001] (Table 3.3). Figures 3.6, 3.7 and 3.8 show a series of two-beam bright field images of the sample deformed up to $9 \%$ strain and the analysis of the dislocation types is shown in Tables 3.4, 3.5 and 3.6 respectively. In Figure 3.6, it can be seen that dislocations B and D are clearly visible when $\mathbf{g}=(020)$ and $\mathbf{g}=(\overline{200})$. This rules out the possibility that these dislocations can be of the $<100\rangle$ type. By analyzing the images obtained with other $\mathbf{g}$ conditions it is possible to identify dislocation $\mathrm{D}$ as $\mathbf{b}=[\overline{110}]$ from the fact that it is visible in all of the two-beam conditions considered. Dislocation $\mathrm{F}$ has also been identified to be $\mathbf{b}=[\overline{101}]$ and appears to be formed by the junction of a [100] and [001] dislocation, namely E and G. Similarly, in Figure 3.7 dislocation B is visible in both the $\mathbf{g}$ $=(0 \overline{2} 0)$ and $\mathbf{g}=(\overline{2} 00)$ diffraction conditions, and hence, is either a $\langle 110\rangle$ or a $\langle 111\rangle$ type dislocation. In Figure 3.8, dislocations A and B are invisible in two diffracting conditions (101) and $(0 \overline{2} 0)$ and are, thus, identified to have a Burgers vector, $\mathbf{b}=[\overline{101}]$. The dislocation morphology shows a number of straight segments with some kinks as well as tangles. By considering the trace direction of the dislocation segments in two different beam directions, it is possible to determine the line direction [27]. In the case of the $<100>$ dislocations, the line directions are frequently near $<111>$ directions, i.e. these segments are found to be of the mixed character. A similar morphology has also been observed in previous studies carried out on CoTi and CoZr [12, 14]. In some of the 
a)

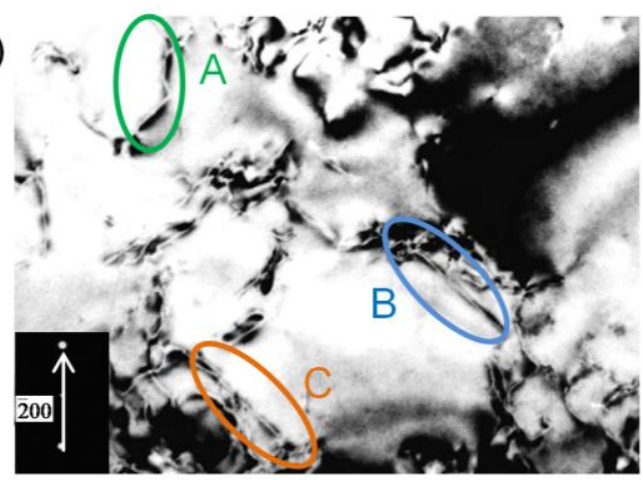

c)

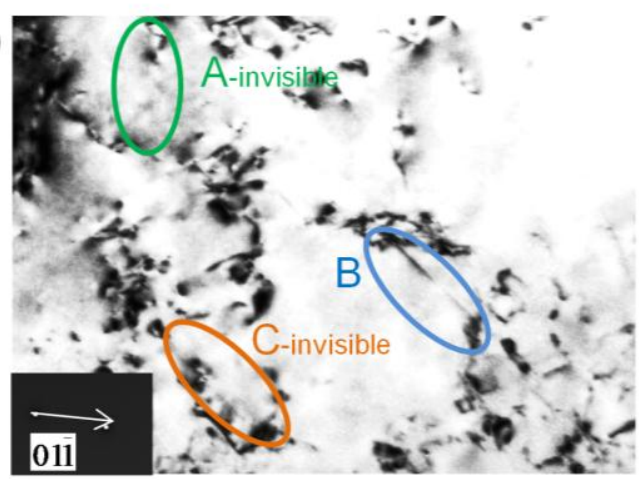

e)

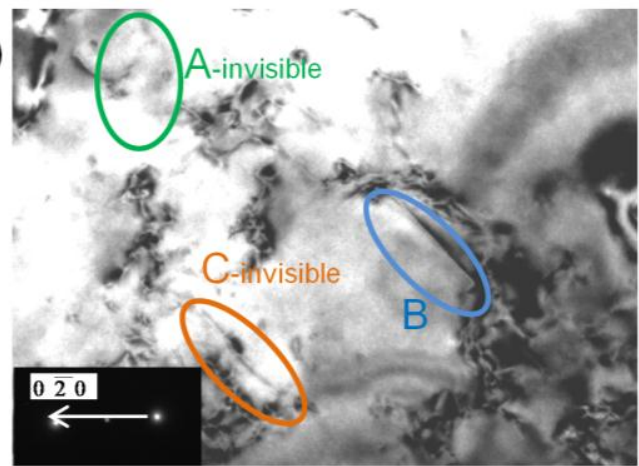

b)

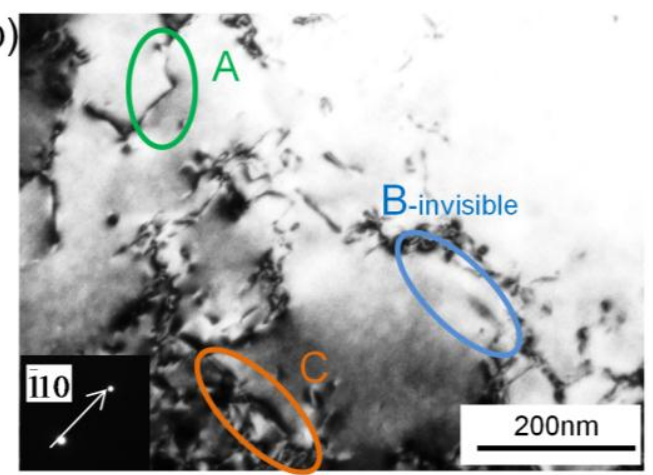

d)

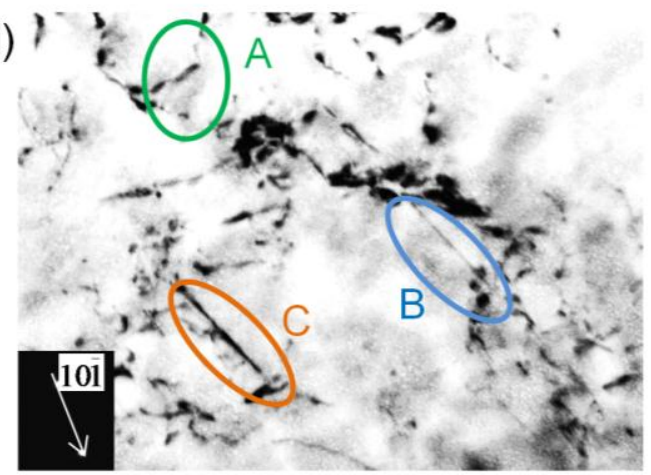

Figure 3.7: Series of two-beam bright field images of dislocations in CoTi deformed to $9 \%$ strain. The electron beam direction is near [001] for images $a, b$, e and near [111] for images c and d. Analysis is shown in Table 3.5.

Table 3.5: Visibility-invisibility analysis of the dislocations in Figure 3.7

\begin{tabular}{|c|c|c|c|c|c|}
\hline $\mathbf{b} \backslash \mathbf{g}$ & $\overline{2} 00$ & $\overline{110}$ & 011 & $10 \overline{1}$ & $0 \overline{2} 0$ \\
\hline A [100] & visible & visible & invisible & visible & invisible \\
\hline B [110/111] & visible & invisible & visible & visible & visible \\
\hline C [100] & visible & visible & invisible & visible & invisible \\
\hline
\end{tabular}



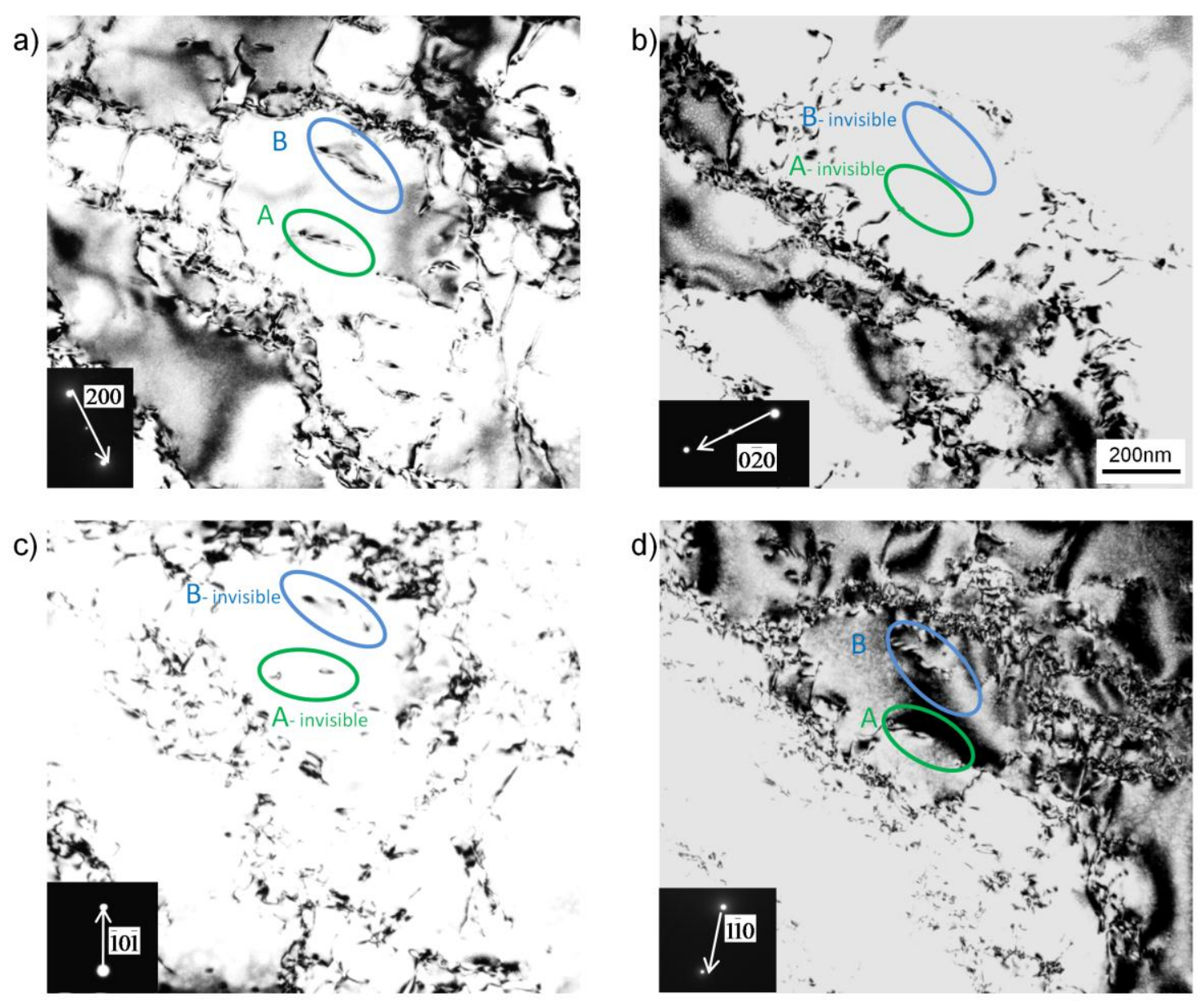

Figure 3.8: Series of two-beam bright field images of dislocations in CoTi deformed to $9 \%$ strain. The electron beam direction is near [001] for images $a, b, d$ and near [111] for image c. Analysis is shown in Table 3.6.

Table 3.6: Visibility-invisibility analysis of the dislocations in Figure 3.8

\begin{tabular}{|c|c|c|c|c|}
\hline $\mathbf{b} \backslash \mathbf{g}$ & 200 & $0 \overline{2} 0$ & $10 \overline{1}$ & $1 \overline{10}$ \\
\hline A [ $[\overline{101}]$ & visible & invisible & invisible & visible \\
\hline B [ $[\overline{101}]$ & visible & invisible & invisible & visible \\
\hline
\end{tabular}




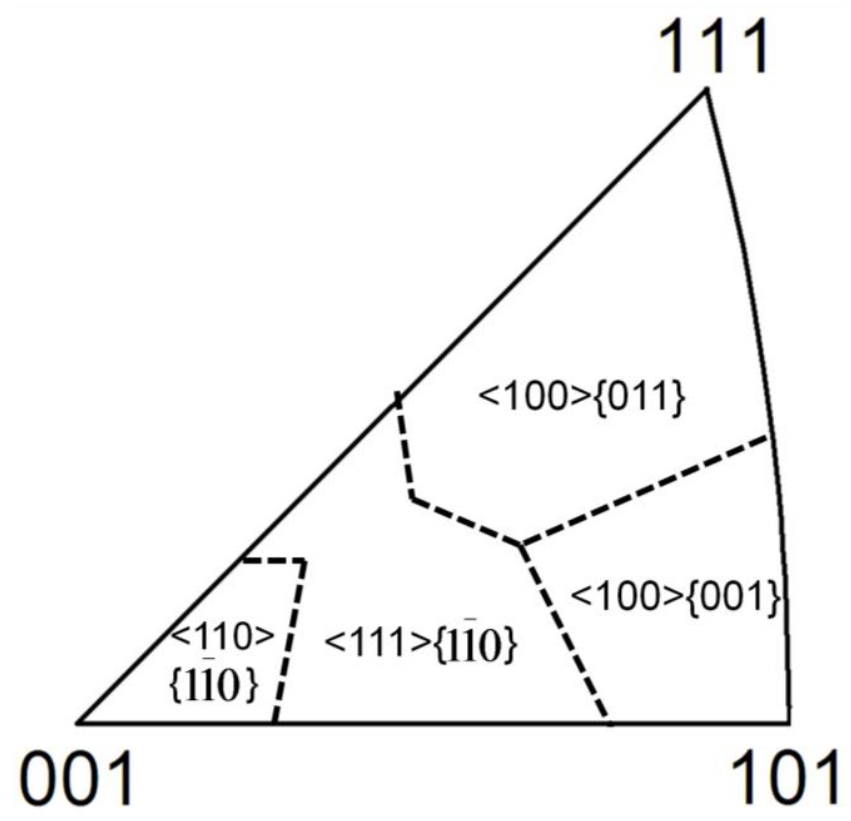

Figure 3.9: Preferred slip mode in B2 alloys based on schmid factor. All slip modes are assumed to have the same critical resolved shear stress

analyzed grains, the operative slip system was in agreement with the preferred slip system based on Schmid factor (Fig.3.9), but in some grains, a discrepancy was seen.

This can be explained by the fact that the post deformation loading axis might have changed considerably compared to the initial loading axis due the rotation of grains under the action of slip. The EBSD data (Fig.3.11b and 3.13b) show that the grains develop significant misorientations associated with the generation and motion of geometrically necessary dislocations. Therefore, it is reasonable to suppose that some grains (or regions within grains) are far from their initial orientation. Additionally, the grain is surrounded by neighboring grains, which place constraints that enforce a different stress state than that imposed on the polycrystalline aggregate. This makes a slip system prediction based upon Schmid factors (based upon macrostresses) of limited value, especially in materials 
with strong single crystal plastic anisotropy, since the developed intergranular stresses can be quite large. No evidence of martensitic phase transformations or twinning was observed.

\subsubsection{EBSD analysis of the deformation modes}

The orientation image map (OIM) of a CoTi tensile sample (inverse pole figure (IPF) coloring scheme) that failed at $2 \%$ strain (before the transition) is shown in Figure 3.10.

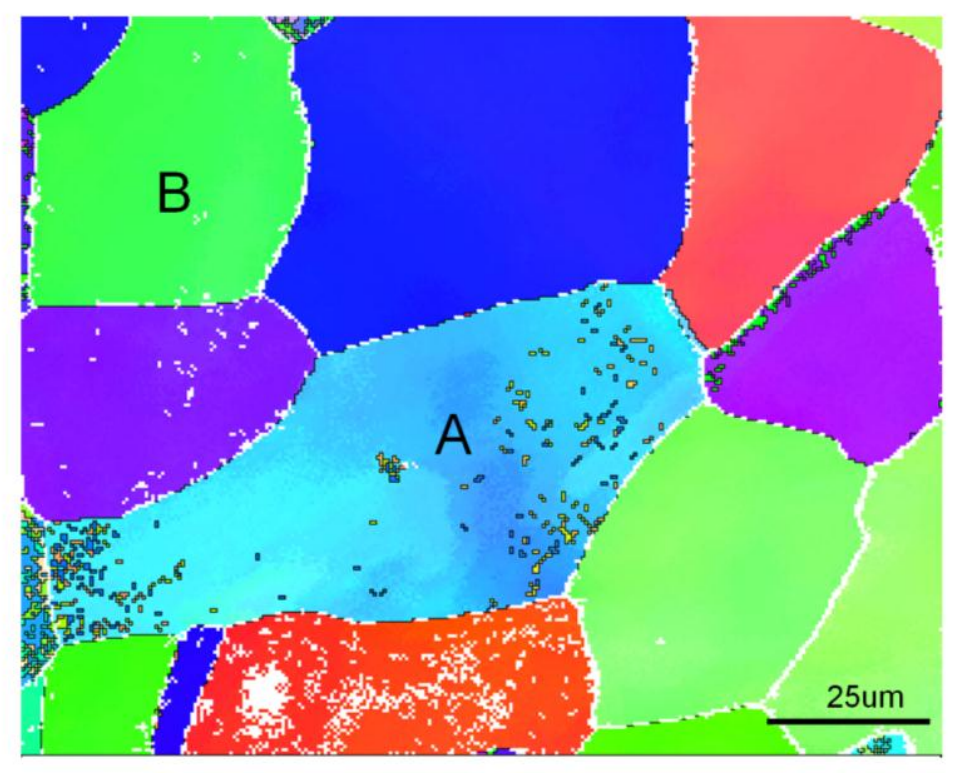

Figure 3.10: OIM of CoTi tensile sample which failed prior to the transition in strain hardening.

Figure 3.11a shows the IGMA distribution from two grains marked A and B and Figure $3.11 \mathrm{~b}$ shows the orientation of the tensile axis of these grains on an inverse pole figure. The maximum and minimum intensities are indicated on each figure. Grain A shows an IGMA corresponding to the $\langle 100>\{011\}$ slip mode while Grain B shows an IGMA corresponding to the $\langle 100\rangle\{001\}$ slip mode (Fig.3.11 and Table 3.2). Of the 22 grains analyzed in this strain regime $32 \%$ of the grains showed an IGMA corresponding 
to the $\langle 100\rangle\{011\}$ slip mode and $50 \%$ of the grains showed an IGMA corresponding to the $\langle 100>\{001\}$ slip mode (Table 3.7). $18 \%$ of the grains showed an IGMA that did not directly correspond to any of the slip modes considered and could not be deconvoluted.

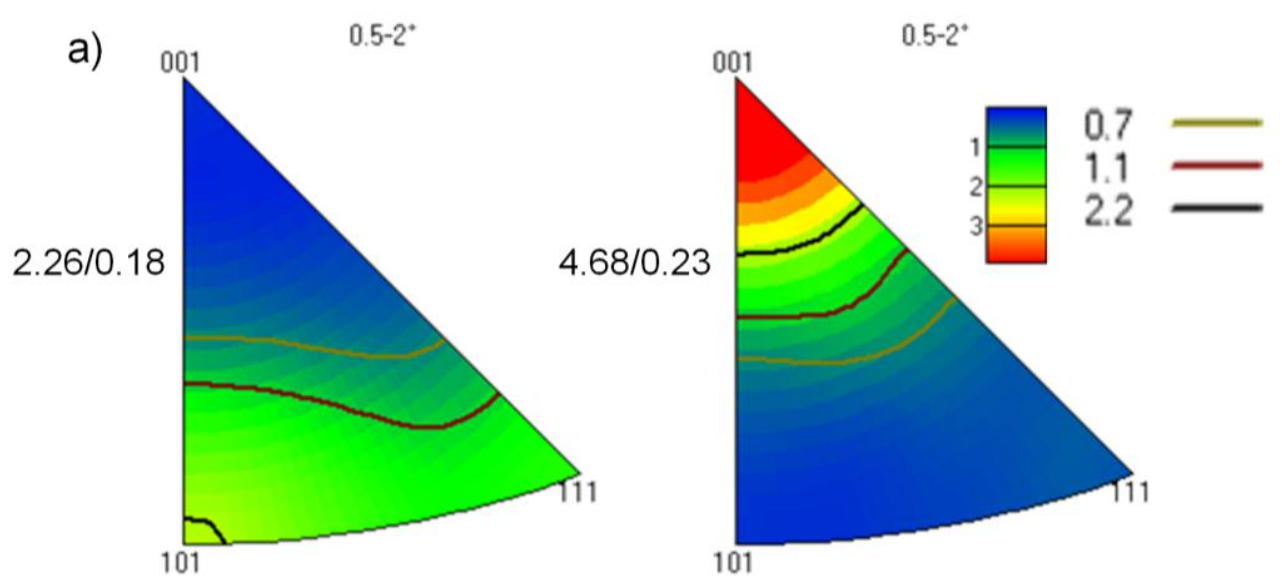

b)

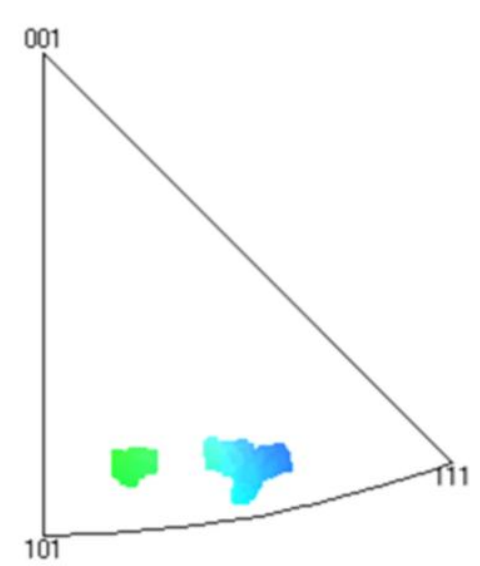

Figure 3.11: Irreducible stereographic triangle showing, a) the in-grain misorientation axis for grains A and B (Fig.3.10) respectively. The maximum and minimum intensities for each plot are indicated. b) The orientation of the loading axis in grains $\mathrm{A}$ and $\mathrm{B}$. 
In $56 \%$ of the grains that showed a clear IGMA, the slip mode found to be active was in agreement with the one that had the highest Schmid factor (Fig.3.9 and 3.11b). None of the grains showed an IGMA corresponding to the $<111>\{110\}$ slip mode. It is acknowledged that it is not possible to distinguish between the $\langle 100\rangle\{001\}$ and $<110>\{110\}$ slip mode using this technique since they both have an IGMA of the type $<010\rangle$. However, previous TEM work carried out in these strain regimes has only shown the presence of $\langle 100\rangle$ dislocations [12]. Hence, at this strain it is reasonable to assume that an IGMA of the type $<010\rangle$ is due to the $\langle 100>\{001\}$ slip mode. Thus, prior to the transition in strain hardening only cube slip modes are active. Finally, this conclusion is in keeping with prior evidence obtained using in-situ neutron diffraction combined with polycrystal plasticity modeling [7].

Table 3.7: Percentage of grains showing a particular IGMA at two different strain levels

\begin{tabular}{|c|c|c|c|}
\hline Slip system & IGMA & $\begin{array}{c}\text { \% grains (2 \% strain) } \\
\text {-prior to transition }\end{array}$ & $\begin{array}{c}\text { \% grains (16 \% } \\
\text { strain)-beyond the } \\
\text { transition }\end{array}$ \\
\hline$<001>\{110\}$ & $<1 \overline{10}>-6$ variants & 32 & 43 \\
\hline $\begin{array}{c}<001>\{100\} \\
\text { or } \\
<110>\{1 \overline{1} 0\}\end{array}$ & $<010>-3$ variants & 50 & 23 \\
\hline$<111>\{1 \overline{10}\}$ & $<11 \overline{2}>-12$ variants & 0 & 17 \\
\hline N/A & No clear IGMA & 18 & 17 \\
\hline
\end{tabular}




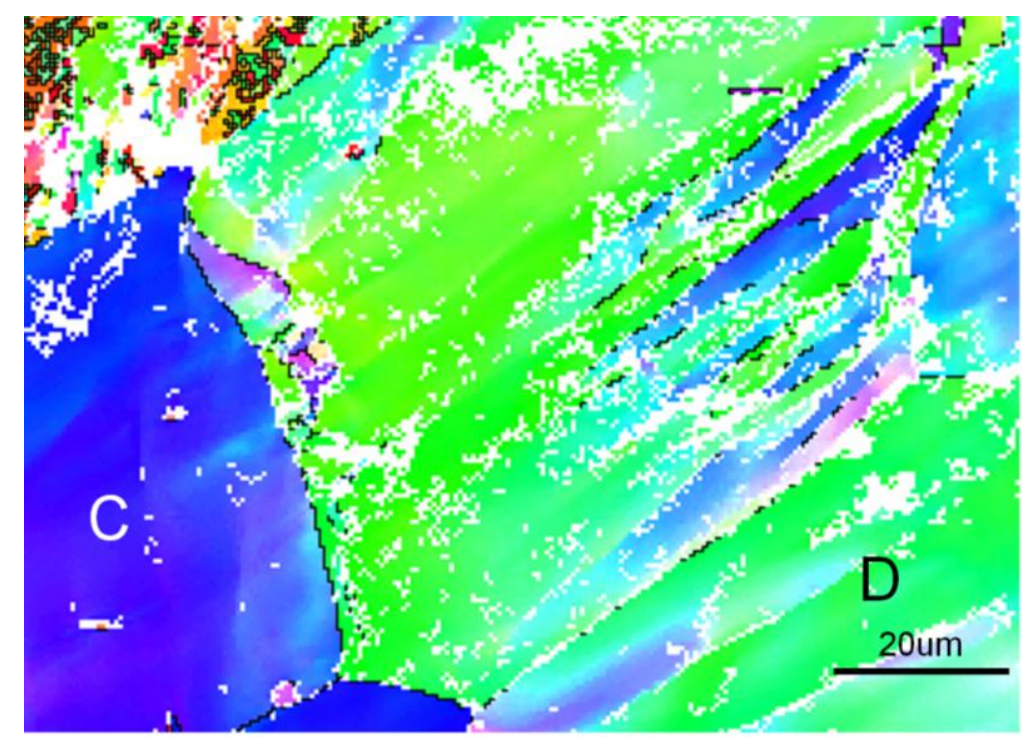

Figure 3.12: OIM of CoTi compression sample deformed beyond the transition in strain hardening.

The OIM (IPF coloring scheme) of a CoTi compression sample that failed at $\sim 16 \%$ strain (beyond the transition) is shown in Figure 3.12. Figure 3.13a shows the IGMA distribution from two grains marked $\mathrm{C}$ and $\mathrm{D}$ and Figure $3.13 \mathrm{~b}$ shows the orientation of the tensile axes of these grains on an inverse pole figure. The maximum and minimum intensities are indicated on each figure. Of the 30 grains analyzed in this strain regime 17 $\%$ of the grains show a dominant $<11 \overline{2}>$ IGMA which corresponds to the $<111>\{1 \overline{10}\}$ slip mode (Table 3.7). For example, Grain C shows two dominant IGMA's, one corresponding the $\langle 100\rangle\{011\}$ slip mode and one corresponding to the $<111>\{1 \overline{10}\}$ slip mode. Grain D shows an IGMA corresponding to the $<111>\{1 \overline{10}\}$ slip mode (Figure 3.13 and Table 3.2 ). Again, since the IGMA due to the $<110>\{1 \overline{10}\}$ and $<100>\{001\}$ slip modes is the same, it is not possible to unambiguously determine whether $\langle 110>\{1 \overline{10}\}$ slip is present. In samples strained to lower levels, the intensity of 
the dominant IGMA is higher than in samples with large strains (Figs. 3.11 and 3.13). [Again, no evidence of martensitic phase transformations or twinning was seen.]
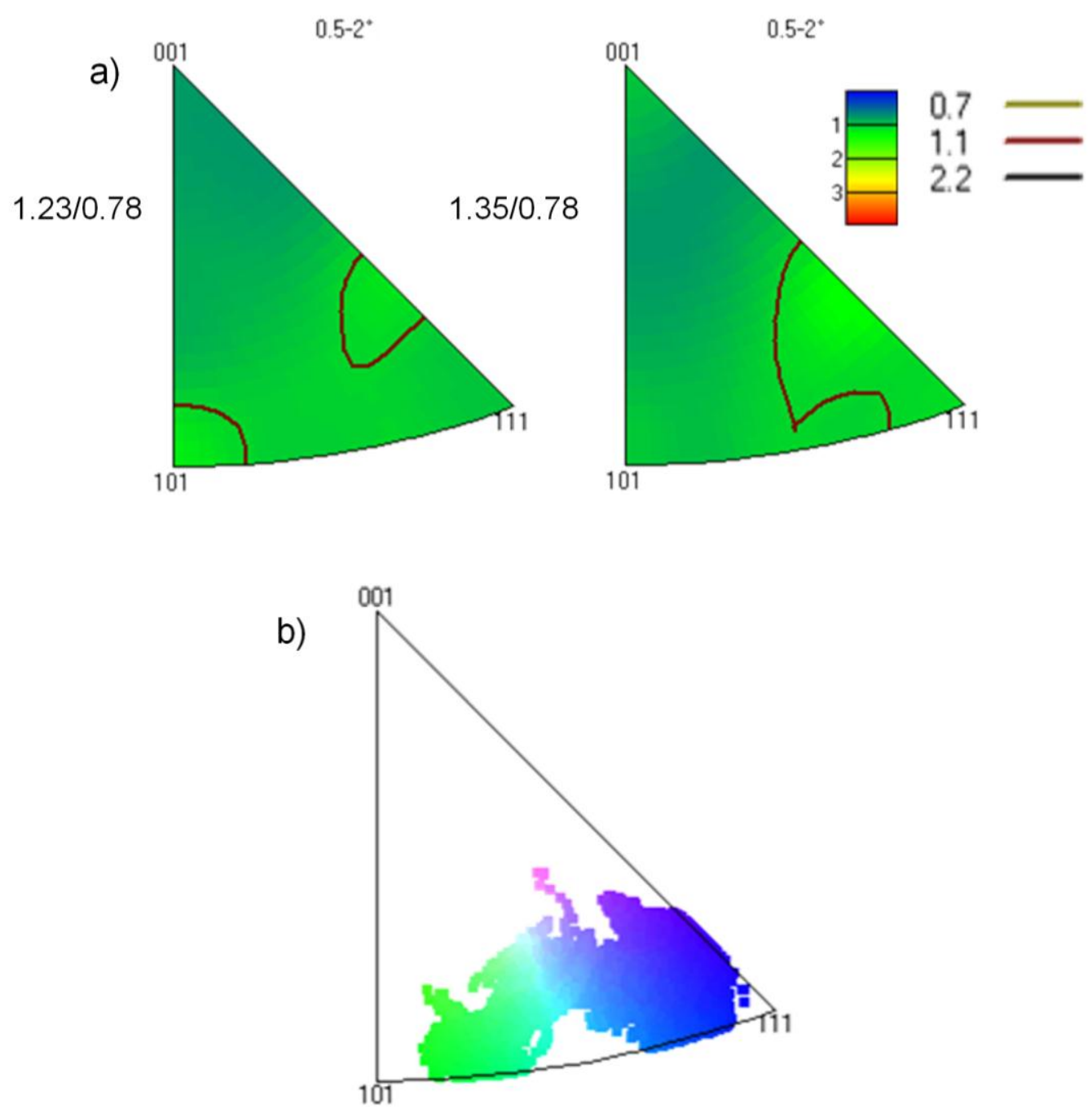

Figure 3.13: Irreducible stereographic triangle showing, a) the in-grain misorientation axis for grains $\mathrm{C}$ and $\mathrm{D}$ (Fig.3.12) respectively. The maximum and minimum intensities for each plot are indicated. b) The orientation of the loading axis in grains $\mathrm{C}$ and $\mathrm{D}$. 


\subsection{Discussion}

The present results ( $\mathbf{g} \cdot \mathbf{b}$ and IGMA analysis) suggest the activity of both the $<110>\{1 \overline{10}\}$ and $<111>\{1 \overline{10}\}$ slip modes in CoTi, but only at strains beyond the transition in strain hardening. The presence of either of these slip modes would provide 5 independent slip systems, and hence, provide a satisfactory explanation for the anomalous ductility of this B2 compound. As shown by Wollmershauser, et al. [7], when only the $\langle 100>\{011\}$ slip mode is present, the stress builds up at a very fast rate and such high stress levels can accelerate fracture. On the other hand, the activation of a secondary slip mode makes plastic deformation easier and promotes a delay in fracture. It would not represent a strict satisfaction of the von Mises criterion, which calls for 5 independent "easy" slip systems, since the $<110>$ and $<111>$ type slip systems obviously have a higher critical resolved shear stress than the $<100>$ "cube" type slip systems.

\subsection{1 $\mathrm{g} \cdot \mathrm{b}$ analysis}

Previous TEM studies done on CoTi did not reveal the presence of dislocations other than those with a $<100>$ Burgers vector $[12,28]$. One of the reasons for this could be that all these samples were strained to only $\sim 2 \%$ and the present results show that the $\langle 110\rangle$ and $<111>$ dislocations appear to be active only at higher strains (and stresses). $<110>$ dislocations have been observed in very few B2 alloys deformed at room temperature (see review [4].) <110> dislocations have been seen in NiAl deformed at room temperature [29], but molecular dynamics calculations with embedded-atom potentials suggest that they are not mobile [30]. Several researchers have reported the presence of $<100\rangle,\langle 110\rangle$ and $<111>$ dislocations in $\mathrm{B} 2 \mathrm{RuAl}$ alloys, (which has the highest melting

point of all B2 aluminides) [31- 34]. Consistent with this observation of 5 independent 
slip systems, RuAl is reported to have a high "chisel toughness" [35]. However, no dissociation of these dislocations has been observed. A few $\langle 110\rangle$ dislocations have also been observed in B2 CoZr. In the case of CoZr, $\langle 110\rangle$ dislocations were found at junctions with $<100>$ dislocations [14], and the authors speculated that they were formed by these junctions and are sessile. In the present work, some of the $\langle 110\rangle$ dislocations form junctions with <100> dislocations (Fig.3.6) and while this junction may serve as a source mechanism, we hypothesize that slip in these alloys may actually be mobility rather than source limited. The aforementioned neutron diffraction results suggest that the hard dislocation mechanisms are glissile (albeit with a high CRSS.)

The observation of $\langle 111\rangle$ dislocations is surprising in light of theoretical predictions [36] which suggest the operation of only $<100>$ type slip in CoTi. Although the $<111>$ APB energy for CoTi is not predicted to be as high as $\mathrm{NiAl}\left(900 \mathrm{~mJ} / \mathrm{m}^{2}\right.$ [37], the calculated value of $553 \mathrm{~mJ} / \mathrm{m}^{2}$ [36], it is still considered high. Historically, it has been held that <111> slip only occurs in B2 alloys with a low antiphase domain boundary (APB) energy. However, recent studies of RuAl have revealed the presence of <111> dislocations [33, 34]. Similarly, some TEM [9] evidence of $<111>$ slip has been reported in the ductile rare-earth B2 family which are predicted to have a high APB energy. Although the focus of this paper is on CoTi, similar work by the authors on CoZr has also revealed $<111>$ dislocations in CoZr deformed past the transition in strain hardening (see Fig. 3.14). In light of these findings, the present observation of $\langle 111\rangle$ type dislocations in CoTi is simply one more in a growing list of exceptions to the rule. 
a)

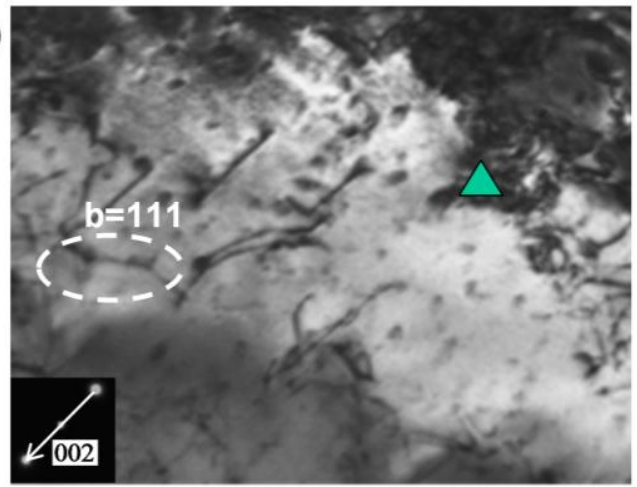

c)

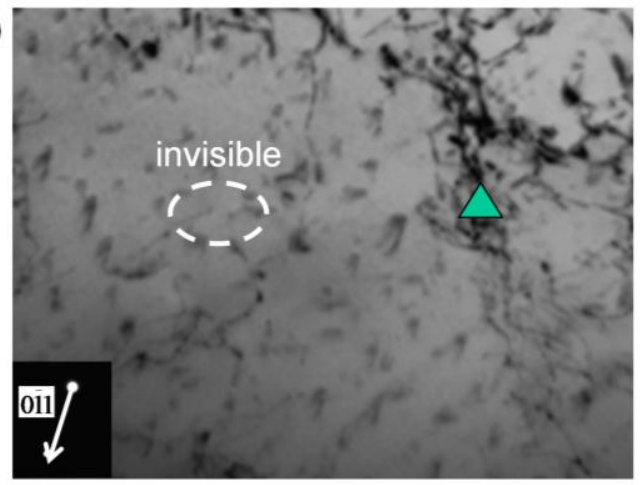

b)

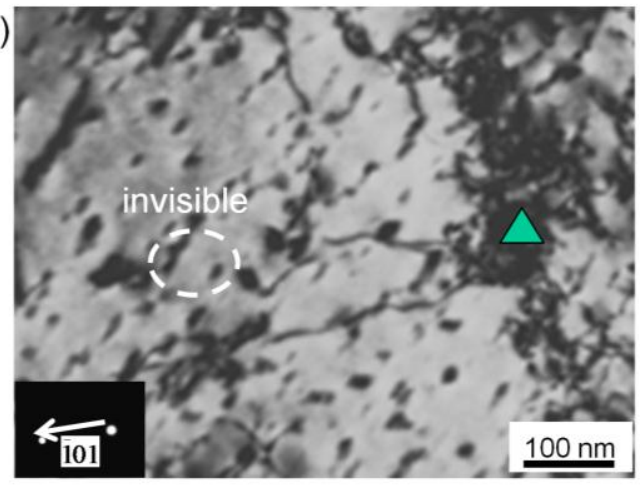

d)

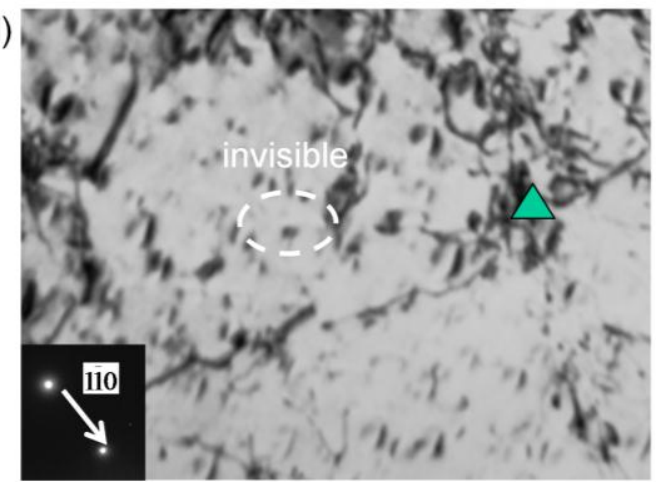

Figure 3.14: Series of two-beam bright field images of dislocations in CoZr

deformed to $20 \%$ strain. The electron beam direction is near [110] for image a and near [111] for image $b, c$ and $d . g \cdot b$ analysis shows the presence of $a$ [111]

\section{dislocation.}

\subsubsection{IGMA analysis}

A weak $<112>$ IGMA corresponding to the $<111>\{110\}$ slip mode has been observed (Fig.3.13a). A weak IGMA generally points to the activity of a number of slip systems [24]. If dislocations of multiple slip systems are present within a grain, the resultant IGMA is the weighted sum of the IGMA's of the different slip systems [23] and this lowers the certainty of determining the individual slip system activities. In fact, the problem of deconvolving dislocation content from EBSD-based misorientation data has 
been extensively treated by others [38-41]. In the present case, the mere occurrence of a weak IGMA (see Figs. 3.12 and 3.13) is indicative of hard slip mode activation because the cube $<100>$ slip mode is not expected to involve the simultaneous activation of many slip systems. Also, the orientation spread within a single grain is much higher at the higher strain levels. The spread is so large that it is likely that different slip systems and even slip modes are active within different parts of the grain. Notably, the intensity of the IGMA distributions increased somewhat, if smaller regions within the grains were considered.

\subsubsection{Source and mobility of dislocations}

The present $\mathbf{g} \cdot \mathbf{b}$ and IGMA analysis as well as previous neutron diffraction and crystal plasticity modeling [7] together strongly suggest the activity of $\langle 110\rangle$ and/or $<111>$ slip in CoTi. Previous studies have discussed dislocation sources and mobilities in B2 alloys $[42,43]$. In the case of isotropic elasticity, the energy of a $<111>$ dislocation is three times while that of a $\langle 110>$ dislocation is two times higher than a $<100>$ dislocation. However, in B2 alloys where the anisotropy parameter is greater than one $\left(\mathrm{A}=2 \mathrm{C}_{44} /\left(\mathrm{C}_{11}-\mathrm{C}_{12}\right)\right)$, after accounting for elastic anisotropy, the energy difference between a $\langle 111\rangle$, $\langle 110\rangle$ and $\langle 100\rangle$ screw dislocation is reduced, for e.g., in FeAl the energy of a $<111>$ screw dislocation is only 1.9 times the energy of a $<100>$ screw dislocation [36]. Therefore, <111> dislocation formation would actually lower the energy with respect to the $3<100>$ dislocations which would be required to form it and the same notion is true for $\langle 110\rangle$ dislocations. In $\mathrm{CuZn}$, where the elastic anisotropy is very large, a $<111>$ screw dislocation is actually energetically more favorable than a $<100>$ screw dislocation [36]. 
Using the elastic constants given by Yasuda et al. [44] and the anisotropic elastic selfenergy analysis given by Hirth and Lothe [45], the energy of a $<111>$ screw dislocation was found to be 2 times higher and the energy of a $<110>$ screw dislocation was found to be 1.5 times higher than a $\langle 100\rangle$ dislocation in CoTi. Thus, formation of $\langle 110\rangle$ and $<111>$ dislocations by the combination of $\langle 100\rangle$ dislocations will be energetically favorable in these cases and such an attractive junction could serve as a source mechanism for $\langle 110\rangle$ and $\langle 111\rangle$ dislocations. However, if these hard slip modes are source limited, the alloys with higher anisotropy (energetically more favorable to form $<110>$ and $<111>$ dislocations and hence more sources) would be expected to be more ductile than the more isotropic alloys. In reality, some of the most ductile B2 alloys like $\mathrm{CoZr}, \mathrm{AgY}$ and $\mathrm{CuY}$ are nearly isotropic. Hence, it appears that the choice of slip direction is not source, but mobility limited. Nevertheless, this analysis of the self-energy does provide an explanation for TEM observations of $\langle 111\rangle$ and $\langle 110\rangle$ dislocations at junctions in elastically anisotropic B2 alloys, even when such dislocations are observed to be sessile. (In order to contribute to strain accommodation, these dislocations must be present in the microstructure, but their presence does not prove their role in strain accommodation.)

As a result, complementary techniques like in-situ neutron diffraction and crystal plasticity modeling are very useful, since they reveal information about the strain accommodating mechanisms. When there is no energetic driving force for junction formation (which is the case in isotropic alloys like CoZr), observation of large Burgers vector dislocations is more strongly suggestive of their role in the deformation. The present TEM/EBSD study reinforces prior conclusions that a hard slip mode(s) is 
responsible for the anomalous ductility of select B2 compounds because it did not reveal any alternative mechanisms, such as mechanical twinning or stress induced martensite. In ductile B2 intermetallics, like $\mathrm{CuZn}$, where deformation takes place by the slip of <111> dislocations, the planar dissociation of $\langle 111\rangle$ dislocations is predicted, and hence, they are considered to be more mobile than undissociated $<100>$ dislocations which may have a non-planar core [36]. However, in CoTi, both the $\langle 100\rangle$ and $\langle 111\rangle$ dislocations are predicted to be undissociated and relatively difficult to move (based upon the observed core configurations) [36]. The $<110>$ and $<111>$ dislocations have a large Burgers vector which translates into a high Peierls resistance, compared to the $\langle 100\rangle$ dislocations. However, the critical resolved shear stress (CRSS) for the activation of these secondary slip modes is lower in CoZr and CoTi as compared to the brittle NiAl. <111> slip has also been observed in NiAl [46], but the CRSS is so high that it is unable to delay fracture. On the other hand, in alloys like CoTi and CoZr, the secondary slip systems are activated early enough to increase the ductility in these alloys. CoZr, which has a lower relative CRSS for the secondary slip modes than CoTi [7], shows a higher ductility, consistent with this explanation. A more detailed knowledge of the core structure of <110> and <111> dislocations under stress may provide a more fundamental understanding regarding what enables the activation of early secondary slip in select B2 alloys.

\subsection{Conclusions}

TEM-based $\mathbf{g} \cdot \mathbf{b}$ and EBSD-based IGMA analyses confirm that the dominant slip modes in CoTi are $<100>\{011\}$ and $<100>\{001\}$. However, beyond a transition in strain hardening, secondary slip modes are activated. Prior work has shown that the slip of 
either $<110>$ or $<111>$ dislocations (or both) could explain the observed internal strain developments. The current study shows that both of these types of dislocations are present. A limited amount of $\langle 110\rangle$ or $\langle 111\rangle$ dislocation motion can reduce stress concentrations that could otherwise lead to premature fracture. The presence of the $<111>\{1 \overline{1} 0\}$ slip mode provides five independent slip systems by itself. The $<110>$ $\{1 \overline{10}\}$ and $\langle 100\rangle\{110\}$ (or $\langle 100\rangle\{010\}$ ) slip modes combine to provide five independent slip systems. The verified presence of the hard slip modes (and absence of any other strain accommodating mechanisms like twinning or martensitic phase transformations) provides a satisfying explanation for the ductility observed in CoTi and extends to other anomalously ductile B2 compounds, like CoZr. Based upon the current and prior observations of $<110\rangle$ and $<111>$ dislocations in B2 intermetallic compounds, it is suggested that these dislocations are mobility, rather than source, limited. The present results suggest that future atomistic modeling studies of dislocation dissociations and core structures in B2 alloys should focus on the evaluating the mobility of dislocations which were previously suggested to be sessile.

\subsection{Acknowledgements}

This work was supported by the National Science Foundation through a CAREER Grant DMR-0547981. We are grateful to Mr. Cecil A. Carmichael, Jr. and Dr. Evan Ohriner (Oak Ridge National Laboratory) for performing the arc-casting and hot extrusion of CoTi, respectively. We would also like to thank Mr. Richard White (University of Virginia). 


\subsection{Referenecs}

1. Sauthoff G. Intermetallics, New York: VCH; 1995.p.1-5.

2. Potter DI. Materials Science and Engineering 1970; 5(4): 201-9.

3. Miracle DB. Acta Materialia 1993; 41(3): 649-84.

4. Baker I. Materials Science and Engineering A 1995; 193:1-13.

5. Lall C, Loretto MH, Harris IR. Acta Metallurgica 1978; 26(10):1631-41.

6. Yamaguchi T, Kaneno Y, Takasugi T. Scripta Materialia 2005; 52(1): 39-44.

7. Wollmershauser JA, Neil CJ, Agnew SR. Metall Trans A 2010; 41A(5): 1217-29.

8. Gschneidner K, et al. Nature Materials 2003; 2(9): 587-90.

9. Russell AM, et al. Acta Materialia 2004; 52(13): 4033-40.

10. Kaneno Y, et al. J. of Alloys and Compounds 2008; 456(1-2): 125-34.

11. Takasugi T, et al. Philosophical Magazine A 1990; 61(5): 785 - 800.

12. Takasugi T, Yoshida M, Kawabata T. Philosophical Magazine A 1992; 65(1): 2940.

13. Yoshida M, Takasugi T. Philosophical Magazine A 1993; 68(2): 401-17.

14. Francois A. Veyssiere P. Intermetallics 1994; 2(1): 9-22.

15. Kaneno Y, Takasugi T, Hanada S. Mater Sci Eng A 2001; 302(2): 215-21.

16. Kaneno Y, Takasugi T. Journal of Materials Science 2003; 38(5): 869-76.

17. Jain A. PhD Thesis-Modeling of Twinning in Mg AZ31B, Charlottesville: University of Virginia; 2005. p. 46.

18. Fultz B, Howe JM. Transmission Electron Microscopy and Diffractometry of Materials, $3^{\text {rd }}$ ed. New York: Springer; 2007. p. 359-69.

19. Takasugi T, Izumi O. Journal of Materials Science 1988; 23(4): 1265-73. 
20. Rapperport EJ,Hartley CS. Trans. AIME 1960; 218:869-77.

21. Taylor W, Moore A. Journal of Nuclear Materials 1964; 13(1): 23-7.

22. Chun YB, Davies CHJ. The Evolution of In-Grain Misorientation Axes (IGMA) During Deformation of Wrought Magnesium Alloy AZ31, in: Agnew SR, et al. (Eds.). Magnesium Technology 2010. Warrendale, PA: TMS (The Minerals, Metals \& Materials Society); 2010. p.433-7.

23. Hadorn JP. PhD Thesis-Texture weakening mechanisms of rare earth containing Mg alloys, Charlottesville: University of Virginia; 2010.p.13-20.

24. Chun YB, et al. Metall Mater Trans A 2010; 41A(13): 3473-87.

25. Mulay RP, Clausen B, Agnew SR. Metall Mater Trans A 2011; 42A(1): 60-70.

26. Wilkens M. Physica Stat. Sol. 1970; 2: 359-70.

27. Loretto MH, Smallman RE. Defect Analysis in Electron Microscopy, London: Chapman and Hall Ltd; 1975. p. 15-36.

28. Shindo D, et al. Intermetallics 1995; 3(2): 167-71.

29. Pascoe RT, Newey CWA. Phys. Stat. Sol. 1968; 29: 357.

30. Mills MJ, et al. HRTEM Observation and EAM calculation of dislocation cores in NiAl, in: Baker I, et al. (Eds.). High-Temperature Ordered Intermetallic Alloys V. Pittsburgh, PA: MRS (Materials Research Society); 1993.p. 257-62.

31. Fleischer R, Field RD, Briant CT. Metall Trans A 1991; 22A: 403.

32. Sabariz ALR, Taylor G. Preparation, Structure and Mechanical Properties Of RuAl and (Ru,Ni)Al Alloys, in: Koch CC, et al. (Eds.). High-Temperature Ordered Intermetallic Alloys VII. Pittsburgh, PA: MRS (Materials Research Society); 1997.p. 611-6. 
33. Lu DC, Pollock TM. Acta Materialia 1999; 47(3): 1035-42.

34. Nandy TK, Feng Q, Pollock TM. Scripta Mater 2003; 48(8):1087-92.

35. Fleischer RL, Zabala RJ. Metall.Trans. 1990; 21A: 2709.

36. Paidar V, et al. Intermetallics 2010; 18(7): 1285-87.

37. Fu CL, Yoo MH. First-Principles Investigation of Mechanical Behavior of B2 Type Aluminides: FeAl and NiAl, in: Johnson LA, et al. (Eds.). HighTemperature Ordered Intermetallic Alloys IV. Pittsburgh, PA: MRS (Materials Research Society); 1991.p. 667-72.

38. Sun S, Adams BL, King WE. Philosophical Magazine A 2000; 80(1): 9-25.

39. Kysar JW, Briant CL. Acta Materialia 2002; 50(9): 2367-80.

40. El-Dasher BS, Adams BL, Rollett AD. Scripta Materialia 2003; 48(2):141-5.

41. Pantleon W. Scripta Materialia 2008; 58(11): 994-7.

42. Yoo MH. Materials Transactions JIM 1990; 31(6): 435-42.

43. Veyssiere P, Douin J. Dislocations, in: Westbrook JH, Fleischer RL (Eds.). Intermetallic Compounds: Principles and Practice. Vol.1. New York: John Wiley \& Sons, Ltd.; 1995. p. 519-558 [chapter 22].

44. Yasuda H, Takasugi T, Koiwa M. Materials Transactions JIM 1991; 32(1): 48-51.

45. Hirth JP, Lothe.J. Theroy of Dislocations, $2^{\text {nd }}$ ed. Malabar, FL: Krieger Publishing Company; 1992. p.423-67.

46. Scharnweber M, et al. Journal of physics, Conference series 2010; 240: 012026. 


\title{
4. The fracture behavior of B2 structured MgR
}

\author{
intermetallics $^{i}$ \\ R.P. Mulay and S.R. Agnew \\ Materials Science and Engineering, \\ University of Virginia, Charlottesville, VA 22904-4745
}

\subsection{Abstract}

Recent discovery of ductile B2 intermetallics containing a rare earth (R) element and a transition or simple metal (M) raises questions regarding their fracture behavior. We have compared prototypical B2 intermetallics like NiAl and CuZn with some $\mathrm{MgR}$ compounds, which tend to exhibit brittle cleavage on $\{100\}$ cleavage planes, with the exception of $\mathrm{MgCe}$ which cleaves on $\{111\}$ planes.

Keywords: intermetallics, cleavage plane, rare earth, magnesium, EBSD.

For several decades, intermetallic compounds have been extensively studied since they have many attractive properties like high melting temperature, oxidation resistance, hardness, etc. However, their use as structural materials has been limited by low room temperature ductility. Recently, however, a family of ductile intermetallic compounds was discovered by a group at Iowa State University/Ames Laboratory [1]. These ductile intermetallics are line compounds composed of a rare earth metal $(\mathrm{R})$ and a transition metal (M) and they have the $\mathrm{B} 2(\mathrm{CsCl})$ crystal structure. The most surprising aspect of

\footnotetext{
${ }^{i}$ R. P. Mulay and S. R. Agnew ,"The fracture behavior of B2 structured MgR intermetallics." Scripta Materialia 2009; 61(11): 1036-1039. Reproduced with permission from Elsevier.
} 
this discovery is that plastic deformation in these materials appears to be accommodated by $\langle 100>\{011\}$ slip systems [2], which provide only three independent slip systems (similar to $\mathrm{NiAl}$ ), thus violating von Mises condition. Additionally, the anomalous ductility was obtained without any special testing environment or other contrivances like non-stoichiometry, metastable disorder, doping, etc., previously observed to increase the ductility in intermetallic compounds $[3,4]$. We have elected to investigate the possibility that $\mathrm{B} 2$ compounds composed of a rare earth $(\mathrm{R})$ and a simple metal $(\mathrm{Mg})$ may also exhibit the ductilizing effect, as previously speculated [1].

MgY castings were prepared using high purity magnesium (99.9 wt \%) and high purity yttrium $(99 \%$ ii $)$ at the Oak Ridge National Laboratory. The alloying was performed using a Pt-wire resistive furnace within an inert-gas (Ar) filled glove box with additional oxidation protection provided by small additions of the heavy cover gas, $\mathrm{SF}_{6}$. The magnesium was melted in a mild steel crucible coated with Zircwash $\left(\mathrm{ZrO}_{2}\right)$ and the yttrium was then added to the melt. The molten metal was cast into a cylindrical copper mold yielding castings of $\sim 12 \mathrm{~mm}$ x $100 \mathrm{~mm}$. MgCe and $\mathrm{MgNd}$ alloys were prepared from high purity magnesium $(99.9 \mathrm{wt} \%)$, cerium $\left(99.5 \%{ }^{\mathrm{ii}}\right)$ and neodymium $\left(99.5 \%{ }^{\mathrm{ii}}\right)$, respectively, by induction melting in a purpose-built low vacuum chamber back-filled with Ar gas. The alloying was carried out in a graphite crucible coated with boron nitride.

\footnotetext{
ii All the purities are in weight $\%$ and with respect to all elements in the periodic table. In case of rare earths (especially the lanthanides) it should be noted that purity in atomic \% will be lower due to the high atomic masses of these elements compared to the common metallic impurities.
} 
The compositions of these alloys were analyzed by Northern Analytical using the Inductively Coupled Plasma Mass Spectrometry (ICPMS) technique. In atomic percent, the compositions were determined to be $49.3 \mathrm{Mg} / 50.4 \mathrm{Y}$ for $\mathrm{MgY} ; 50.1 \mathrm{Mg} / 49.8 \mathrm{Ce}$ for $\mathrm{MgCe}$ and $52.0 \mathrm{Mg} / 47.9 \mathrm{Nd}$ for $\mathrm{MgNd}$. After considering the assays of the raw materials and likely additional impurities dependent on the method of melting, lists of selected impurities were determined (Table 4.1). The stoichiometric compounds are known to form the B2 crystal structure [5] and this was confirmed by X-ray diffraction carried out on all the alloys. No second phase peaks were discernible in these materials. Thus, these alloys are single phase, to within the detection limit of this technique which is about 2 vol \%. Studies carried out on ductile members of this family like AgY and CuY have shown that they contain measureable amounts of the $\mathrm{M}_{2} \mathrm{R}$ and $\mathrm{R}_{2} \mathrm{O}_{3}$ phases [6]. Hence, it is important to note that complete absence of second phases or the use of very high purity raw materials are not needed to obtain ductility in this family. Unlike AgY and other ductile MR compounds, the rare earth deficient compound stable at room temperature is $\mathrm{M}_{3} \mathrm{R}$ for both $\mathrm{MgCe}$ and $\mathrm{MgNd}$ (not $\mathrm{M}_{2} \mathrm{R}$ ), which will be present in much smaller amounts at small off-stoichiometry and, hence, may not be detected. Furthermore, $\mathrm{MgY}$ is not a perfect line compound but has some solubility and hence small deviations in stoichiometry will not lead to the formation of other phases.

Table 4.1: Impurities analyzed in MgY, MgCe and MgNd given in parts per million atomic concentration.

\begin{tabular}{|c|c|c|c|c|c|c|}
\hline & Al & W & Cu & Zr & Mn & B \\
\hline MgY & 613 & 32 & 33 & 2232 & & \\
\hline MgCe & 702 & & & & 42 & 69 \\
\hline MgNd & 139 & & & & 36 & 829 \\
\hline
\end{tabular}


Traditionally, a "hammer-test" has been used to distinguish between ductile and brittle intermetallic compounds. The MR compounds were discovered in a similar fashion [1]. We have discovered that all of the MgR compounds studied exhibit brittle cleavage failure during routine laboratory handling. We also observe that the samples break very easily when loaded in bending.

Because of the overall brittle character of the $\mathrm{MgR}$ alloys, compression testing was only carried out on MgY using an Instron universal testing machine with a constant crosshead velocity which yielded an initial strain rate of $10^{-3} / \mathrm{s}$. The sample was cut with a diamond saw and ground to a final rectangular geometry of $10.4 \mathrm{~mm} \times 5.9 \mathrm{~mm} \times 3.7$ $\mathrm{mm}$. Teflon tape was used as a lubricant between the sample and WC compression platens. Strain was calculated from the cross-head displacement. The elastic strains have been subtracted prior to conversion to true stress and true plastic strain in the usual way.

The stress vs. strain data (Fig. 4.1 [7]) shows a small yield point peak, which suggests the possibility of a limited density of mobile dislocations during the onset of plastic deformation. Additionally, essentially linear hardening is interrupted by load drops which suggest the possibility of microcracking during the test. The compression testing carried out on MgY shows that it exhibits some ductility in compression. Nevertheless, the fracture mode is brittle cleavage type failure. 


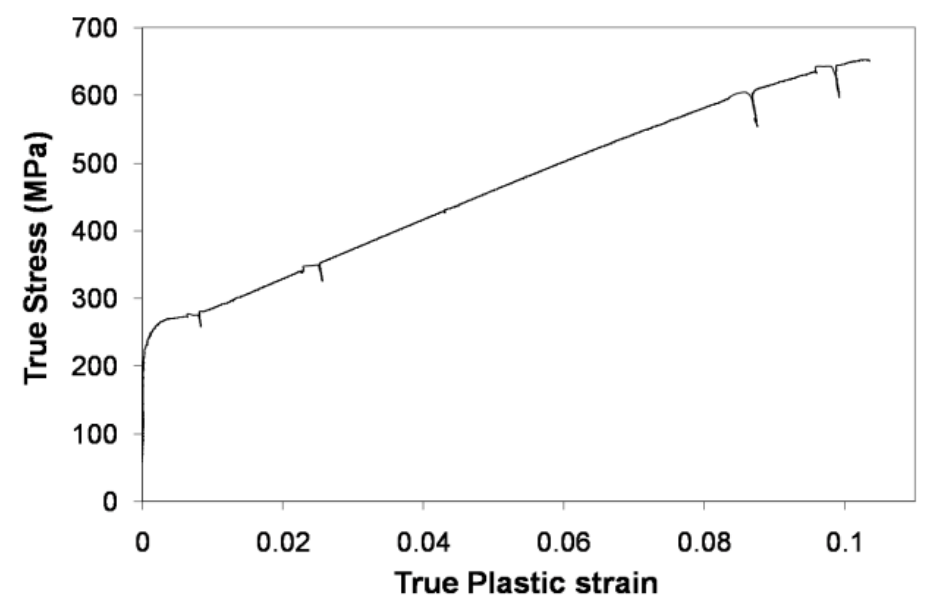

Figure 4.1: Compression test on MgY [7].

SEM fractographic examination shows that the MgR samples undergo primarily cleavage fracture as shown in Figures $4.2 \mathrm{~b}$ and $\mathrm{c}$. The fracture surface is faceted with river like patterns which is typical of cleavage fracture. The fracture surface of these materials has been compared to that of three other B2 compounds (Fig. 4.2), namely polycrystalline $\mathrm{NiAl}$ [8] (an archetypal brittle B2 intermetallic compound), CuZn (a ductile B2 intermetallic) and $\mathrm{AgY}$ (the most ductile member of the newly discovered B2 family). CuZn has a low ordering energy and hence shows the $<111>\{011\}$ slip mode in addition to cube slip, which provides the necessary 5 independent slip systems to satisfy von Mises criterion. CuZn shows evidence of its ductile behavior in the form of microvoid dimples; NiAl exhibits gross intergranular fracture in polycrystalline form; and $\mathrm{AgY}$ appears to exhibit a combination of cleavage (Fig. 4.2e) plus intergranular fracture (Fig. 4.2f [9]). Figure 4.2f is not a micrograph of a fracture surface but of a polished surface of a tensile specimen, and it clearly shows cracking along the grain boundaries. It is speculated that the cleavage stress must be low in $\mathrm{MgR}$ compounds, relative to that 
necessary to activate other fracture modes, whereas AgY appears to resist transgranular cleavage to the extent that other fracture mechanisms are also induced.
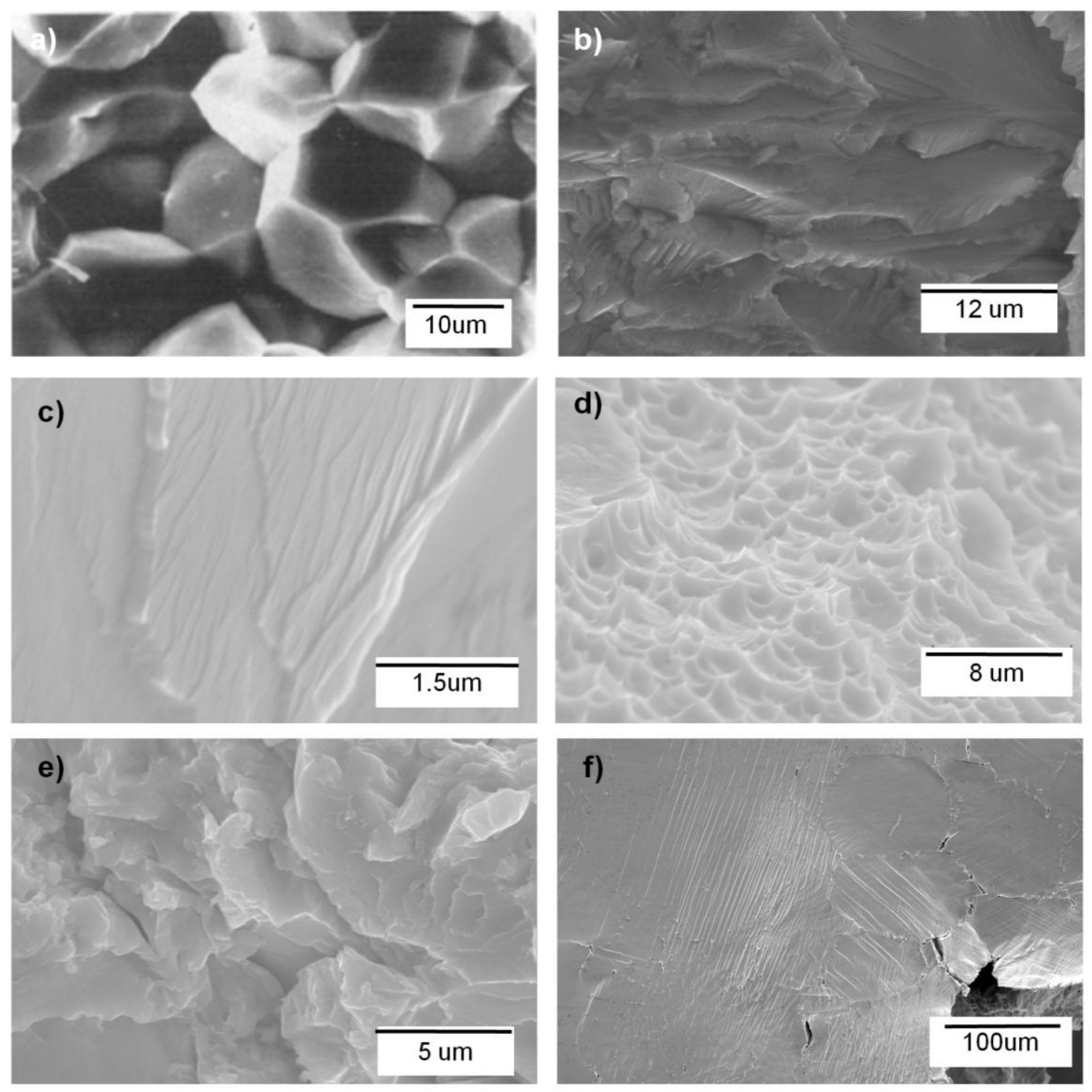

Figure 4.2: Fracture surface of a) $\mathrm{NiAl}[8]$, b) $\mathrm{MgY}$, c) $\mathrm{MgCe}$, d) $\mathrm{CuZn}$ and e)AgY, f) polished surface of a tensile specimen of AgY [9]

Electron backscattered diffraction (EBSD) patterns were obtained from a polished surface adjacent to the fracture surface (denoted Method 1). The sample was prepared by 
grinding with SiC paper up to 1200 grit. This was followed by polishing with 3 um and lum diamond pastes. Finally, the sample was electropolished in an electrolyte composed of $30 \mathrm{ml}$ phosphoric acid and $70 \mathrm{ml}$ ethanol for $\mathrm{MgY}$ and $30 \mathrm{ml}$ nitric acid and $70 \mathrm{ml}$ ethanol for $\mathrm{MgNd}$. The samples were then fractured in bending within a bench vice. Since the sample was fractured after polishing, the possibility of introducing any change to the fracture surface during the polishing process is eliminated. In Method 2, the EBSD patterns were obtained directly of the fracture surface and hence no special sample preparation is necessary $[10,11]$.

A combination of SEM-based stereology (to determine the cleavage facet plane normal vectors with respect to sample coordinates) and EBSD (to determine the grain orientation with respect to the same) have been used to, in turn, characterize the cleavage plane crystallography [12]. The stereology and EBSD measurements are performed with the sample in the same configuration within the SEM without sample replacement to minimize experimental uncertainty [12]. The EBSD system is installed within a JEOL 840 SEM and consists of a Nordlys NL-1 high speed CCD camera and HKL Channel 5.0 software.

Method 1- Automatic beam scan mapping was carried out on the polished surface adjacent to the fracture surface in order to obtain the grain orientation data. The EBSD patterns were obtained at a magnification of $1500 \mathrm{X}$, accelerating voltage of $25 \mathrm{kV}$, probe current of $0.6 \mathrm{nA}$, working distance of $25 \mathrm{~mm}$, and a scan step size of $1 \mu \mathrm{m}$. The experimental set-up is shown in Figure 4.3a. 
Method 2- In this case, the EBSD patterns were obtained directly from the fracture surface [11] and, hence, the fracture surface itself was tilted $\sim 70^{\circ}$ toward the detector. The EBSD patterns were obtained and indexed in the interactive spot mode. For this method, a higher accelerating voltage of $30 \mathrm{kV}$, probe current of $0.6 \mathrm{nA}$, and a working distance of $25 \mathrm{~mm}$ was used. The magnification used varied for each facet depending on the size of the facet. The experimental set-up is shown in Figure 4.3b.

a)

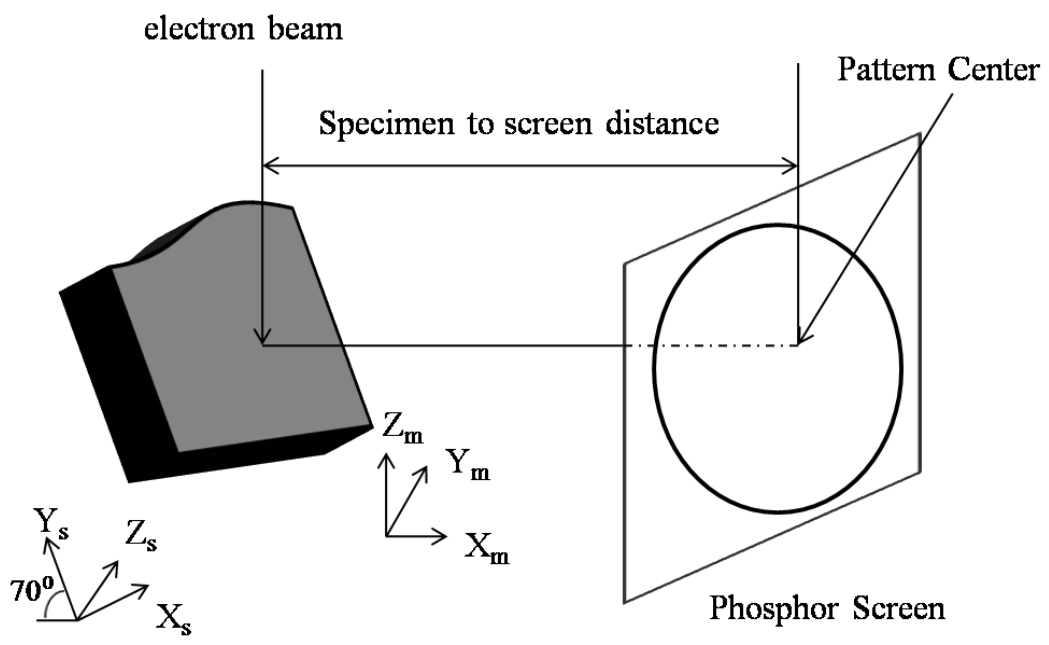

electron beam

b)

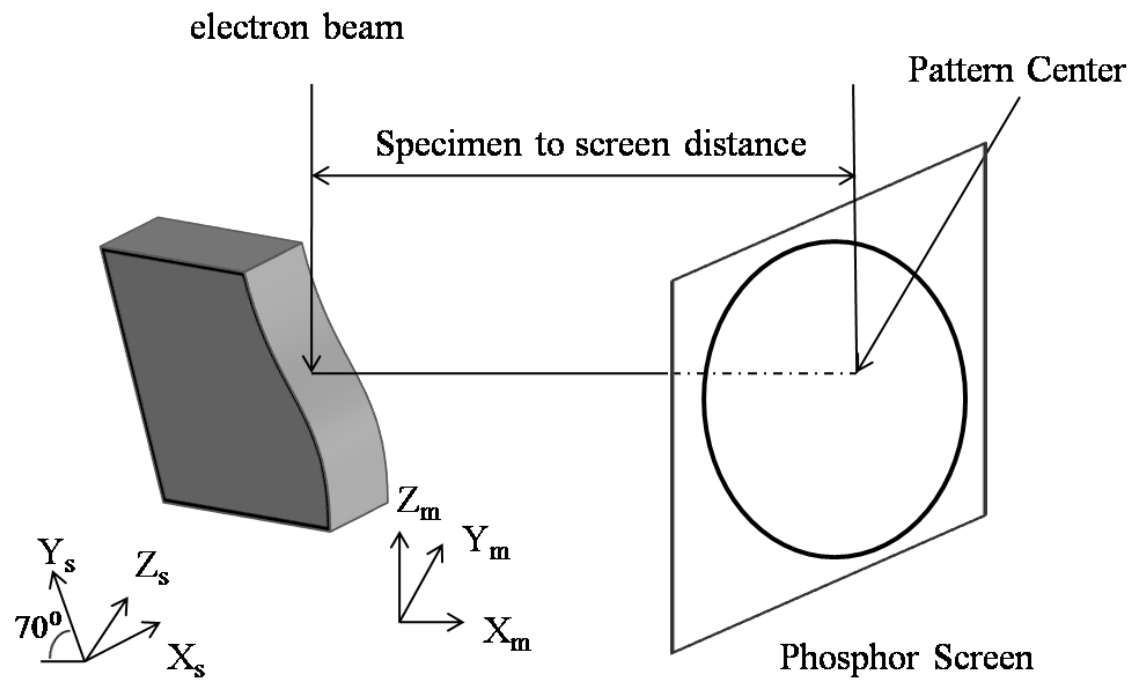

Figure 4.3: EBSD and stereology setup for a) Method 1 (polished surface EBSD), and b) Method 2 (on fracture surface EBSD). 

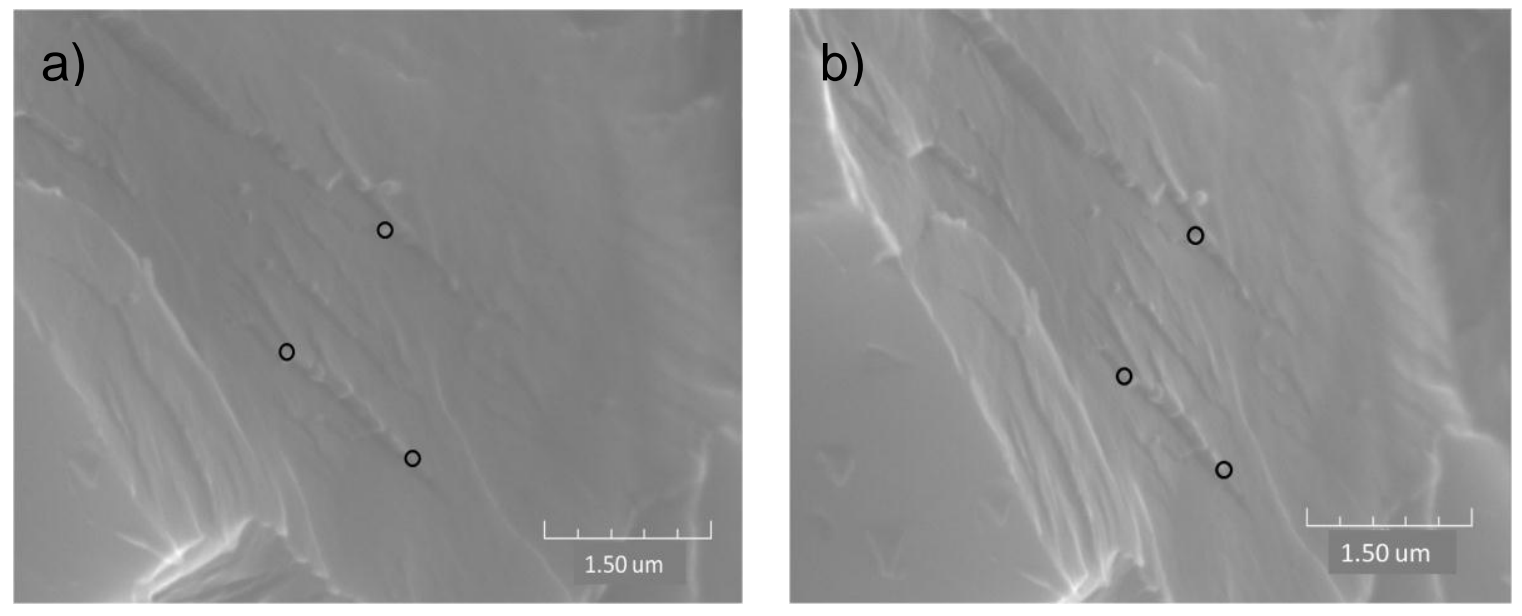

Figure 4.4: SEM micrographs of one of the MgY facets with a sample tilt of a) $60^{\circ}$ and b) $70^{\circ}$

Regardless of whether EBSD Method 1 or 2 was used to obtain the grain orientation information, SEM stereology was used to determine the spatial orientation of the fracture surface facet normals. Stereo image pairs were obtained with the sample first tilted at the $70^{\circ}$ orientation typical of EBSD measurements and then $10^{\circ}$ away from that position at a tilt of $60^{\circ}$. An example of such a stereoimage pair is shown in Fig.4.4, where the circled points are used to define the two vectors necessary to obtain the facet normal. Details of the stereological technique can be found elsewhere [e.g., [13]]. The facet normal was then subjected to a transformation of basis, from sample coordinates to crystal coordinates using the equation, $\mathbf{V}^{\prime}=\mathbf{g V}$, where $\mathbf{V}^{\prime}$ denotes the facet normal vector expressed with respect to crystal coordinates, $\mathbf{V}$ is the same facet normal vector, as measured with respect to sample (microscope or laboratory) coordinates, and $\mathbf{g}$ is the orientation matrix of the grain in which the facet appears. 
The grain orientations obtained using EBSD (Method 1) are represented in orientation image maps (OIMs) superimposed on images of the polished surface adjacent to the fracture surface (Fig.4.5). Again, cleavage-like features are observed.
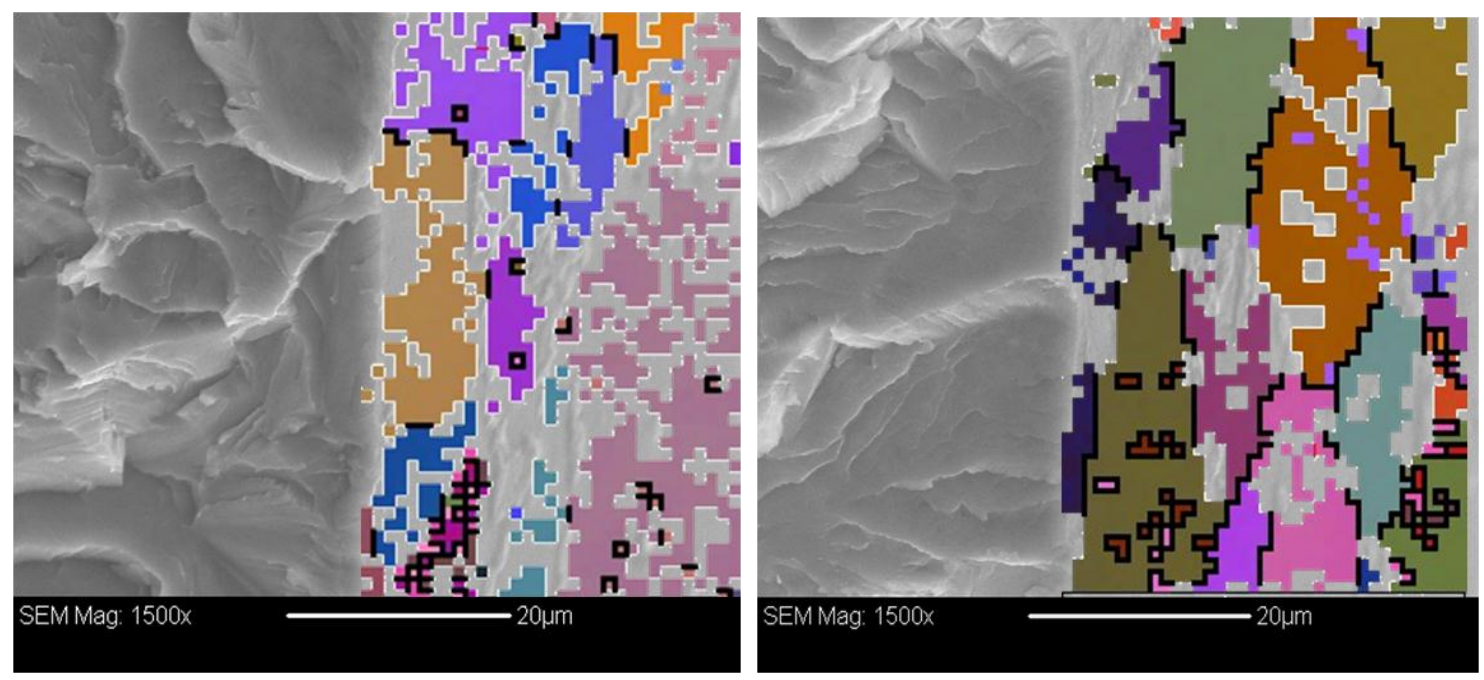

Figure 4.5: Orientation image map superimposed on the polished surface. The fracture surface is faceted with river-like patterns typical of cleavage failure. The analysis described in the text was carried out on the facets adjacent to the polished surface.

The crystallography of fracture surface facets is presented on the irreducible triangular projection in Figure 4.6a and 4.6b. The results indicate that $\mathrm{MgY}$ and $\mathrm{MgNd}$ tend to cleave along $\{100\}$ planes. In case of MgY, 19 specific facet orientations were measured from 9 different grains. 11 of the 19 facets $(58 \%)$ are within $15^{\circ}$ of a $\{100\}$ plane while 3 of the facets $(16 \%)$ are within $15^{\circ}$ of a $\{101\}$ plane. In case of $\mathrm{MgNd}, 15$ specific facet orientations were measured from 3 different grains. 7 of the 15 facets (47\%) are within $15^{\circ}$ of a $\{100\}$ plane while 1 of the facets $(16 \%)$ are within $15^{\circ}$ of a $\{101\}$ plane. None of the measured facets were within $15^{\circ}$ of a $\{111\}$ plane in either $\mathrm{MgY}$ or $\mathrm{MgNd}$, though 
both show some high index cracking. In the case of $\mathrm{MgCe}$, we were unable to obtain a surface suitable for EBSD by mechanical, chemical or electrochemical polishing techniques and, hence, we resorted to method 2. To confirm independence of the results from the method employed, MgY was analysed by both methods. The results determined using method 2 are shown in Figure 4.6c and 4.6d. MgY is again shown to cleave along $\{100\}$ planes (4 of the 6 measured facets are within $15^{\circ}$ of the $\{100\}$ planes.) On the other hand, MgCe tends to cleave on the $\{111\}$ planes (10 of the 12 measured facets within $15^{\circ}$ of the $\{111\}$ planes.)
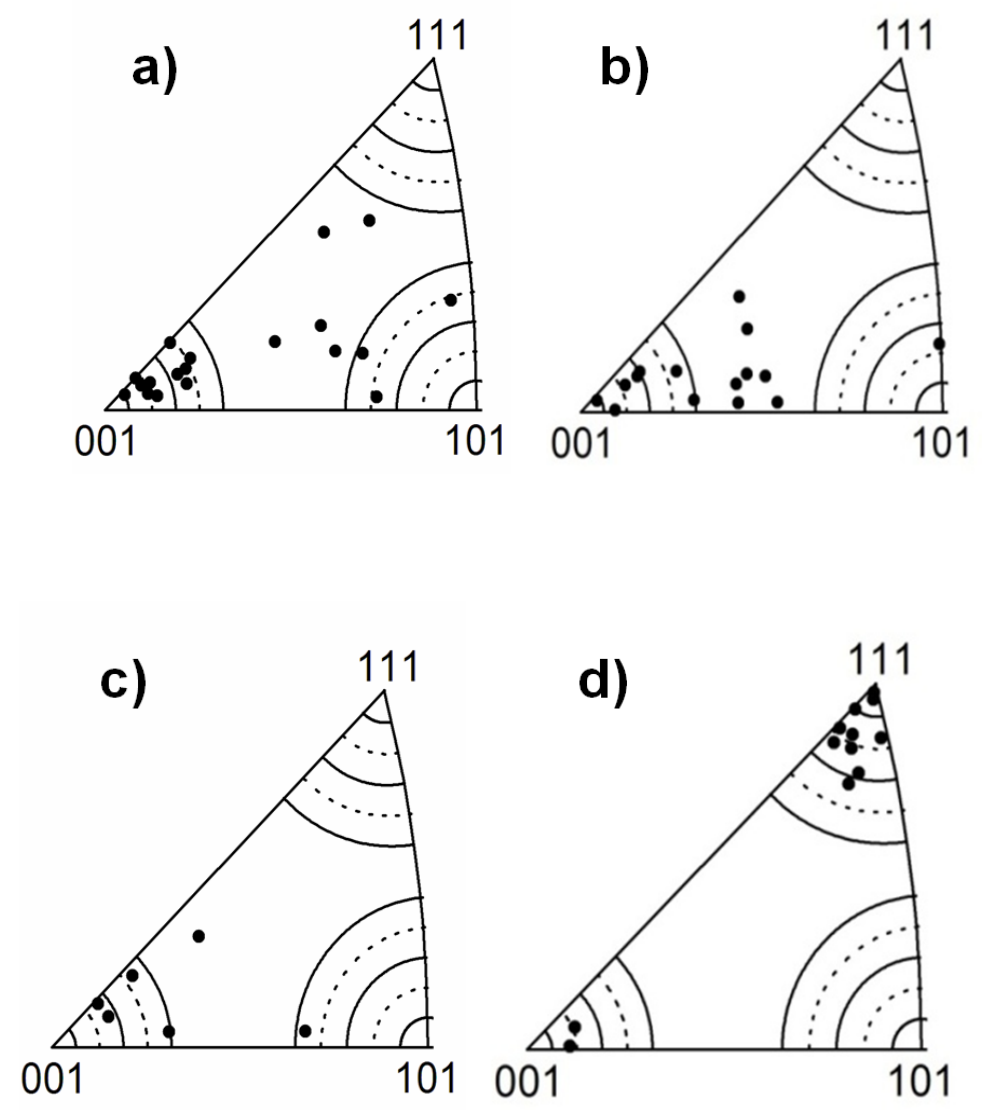

Figure 4.6: Irreducible triangular projection of a) MgY (method1) [7], b) MgNd $(\operatorname{method} 1)$, c) MgY $(\operatorname{method} 2)[7]$ and d) MgCe $(\operatorname{method} 2)$. 
The facet crystallography of AgY was not determined since a surface suitable for EBSD could not be obtained by mechanical, chemical or electrochemical polishing techniques. Neither was method 2 successful, since Kikuchi patterns could not be obtained directly from the fracture surface.

Pugh [14] introduced the concept of using the ratio of bulk to shear modulus $(\mathrm{K} / \mathrm{G})$ as an indicator of ductility, and since then, this criterion has been used by many researchers [e.g., 15-19]. This concept is based on the fact that the bulk modulus is a measure of the intrinsic resistance to atomic bond stretching (required for fracture) while the shear modulus controls the resistance to plastic deformation [20]. Hence, a high value of $\mathrm{K} / \mathrm{G}$ is indicative of ductility and low values correlate with brittleness. Many materials with $\mathrm{K} / \mathrm{G}>1.75$ behave in a ductile fashion $[21,22]$. First principles density functional theory calculations of elastic constants for these $\mathrm{MgR}$ compounds give a value of $\mathrm{K} / \mathrm{G}<$ 1.75 for all the three materials [23] and, thus, they are predicted to be brittle. On the other hand, the generalized gradient approximation calculations give a value of $\mathrm{K} / \mathrm{G}>1.75$ for $\mathrm{MgY}$ and $\mathrm{MgCe}$ and a value of 1.74 for $\mathrm{MgNd}$ [24], suggesting they may be borderline cases. Rice and Thomson [25] have introduced another criterion for ductile vs. brittle behavior which is based on the spontaneous emission of dislocations from an atomically sharp cleavage crack. Based on their analysis, dislocations with wide cores and small values of $\mathrm{Gb} / \gamma(<7.5-10)$ are ductile, where $\mathrm{b}$ is the Burgers vector of the dislocation and $\gamma$ is the surface energy. However, to the author's knowledge the surface energies of MgR intermetallic have not been determined.

On comparison of the fracture surfaces of different B2 compounds, it can be seen that even though $\mathrm{AgY}$ has been shown to exhibit significant tensile elongation to fracture, 
its fracture surface is more similar to the brittle $\mathrm{MgR}$ intermetallics than to that of $\mathrm{CuZn}$, which is characterized by dimples suggestive of microvoid nucleation, growth, and coalescence. Attempts have been made to relate the cleavage plane to ordering energies and surface energies in B2 alloys $[15,26]$. High index fracture planes have been seen in NiAl [27]. While the exact mechanism of formation is not understood, it is proposed that plastic deformation prior to fracture influences their formation. This is supported by the fact that high index fracture planes are absent in NiAl tested at high strain rates (which have very low ductility) [27]. Single crystal NiAl and CoAl (both with B2 crystal structure and high ordering energies) are observed to cleave on $\{110\}$ planes [26], while B2-FeAl [26] and CuZn [28, 29] (both with weaker ordering energies) and most BCC metals cleave along the $\{100\}$ planes [26]. Based upon the scant data listed above, it appears that as the ordering energy decreases, tendency for $\{100\}$ cleavage increases. In contrast with this trend, the MgR compounds have been shown to be highly ordered [6].

It has been reported that when tested in vacuum or nitrogen FeAl cleaves along the $\{111\}$ planes but when tested in an environment containing hydrogen it cleaves along the $\{100\}$ planes $[30,31]$. Tensile tests carried out on FeAl in air and vacuum at strain rates varying from $10^{-6}$ to $1 / \mathrm{s}$ showed a decrease in ductility with decreasing strain rate for samples tested in air but the ductility remained constant when tested in vacuum, indicative of hydrogen induced embrittlement [32]. Pike and Liu showed that only when the strain rate was raised to around $10^{-1 /} \mathrm{s}$ did the results from the tests in different environments (vacuum, oxygen and air) yield similar results [33]. These results indicate that there is insufficient time for the embrittlement process to occur at such high strain rates [33]. It is proposed that aluminum reacts with the moisture in air to form atomic 
hydrogen and it is this hydrogen that causes the embrittling effect. The embrittlement process is composed of several time-dependent steps, for example, the dissociation of water to form atomic hydrogen and the movement of hydrogen into the material [32]. Similar results have been obtained in $\mathrm{Co}_{3} \mathrm{Ti}$ [34].

In the case of the $\mathrm{MgR}$ compounds, it is possible that the cleavage plane is influenced by the environmental effects such as interstitial $\mathrm{H}$ absorbed due to reactions with water vapor. The fact that both $\mathrm{Mg}$ and rare earth elements have a strong affinity for oxygen makes this reaction a realistic possibility. In the present work, slow strain rates of $10^{-3} / \mathrm{s}$ were used for compression tests and in some cases samples failed as they were being clamped in a vice (The hammer test referred to on page 86 was never used in the present study). Although the exact mobility of hydrogen in MgR intermetallics is not known, embrittling effects were observed for FeAl tested at these strain rates [32, 33]. Tensile tests performed at a range of strain rates and corresponding cleavage plane analysis on $\mathrm{MgR}$ alloys will be able to test this hypothesis.

Since chapters 3 and 5 are concerned with anomalously ductile CoTi and CoZr, respectively, the fracture surfaces of both these alloys are shown in Figure 4.7. Similar to $\mathrm{AgY}$, features corresponding to both cleavage and intergranular fracture were seen. The rock-candy appearance indicative of intergranular fracture was dominant compared to the river-like cleavage patterns. The dominance of intergranular fracture agrees with expectations for a material with limited independent easy slip systems (else there is some environmental grain boundary embrittlement mechanism). Chapter 3 has shown that these materials do have hard slip systems that prevent fracture from taking place at the very low strain levels observed for archetypal brittle B2 structured alloys like NiAl. 
The cleavage features in both these alloys are much finer than the $\mathrm{MgR}$ intermetallics. It was not possible to characterize the cleavage plane in these alloys since the cleavage features were not observed adjacent to a surface that could be polished. Hence, method 1 could not used to characterize the cleavage plane. All of the cleavage planes observed were such that even if they were oriented towards the EBSD detector in the SEM, the complex topography of the fracture surface prevented the backscattered electrons from reaching the detector. Hence, method 2 also could not be used to characterize the cleavage plane.
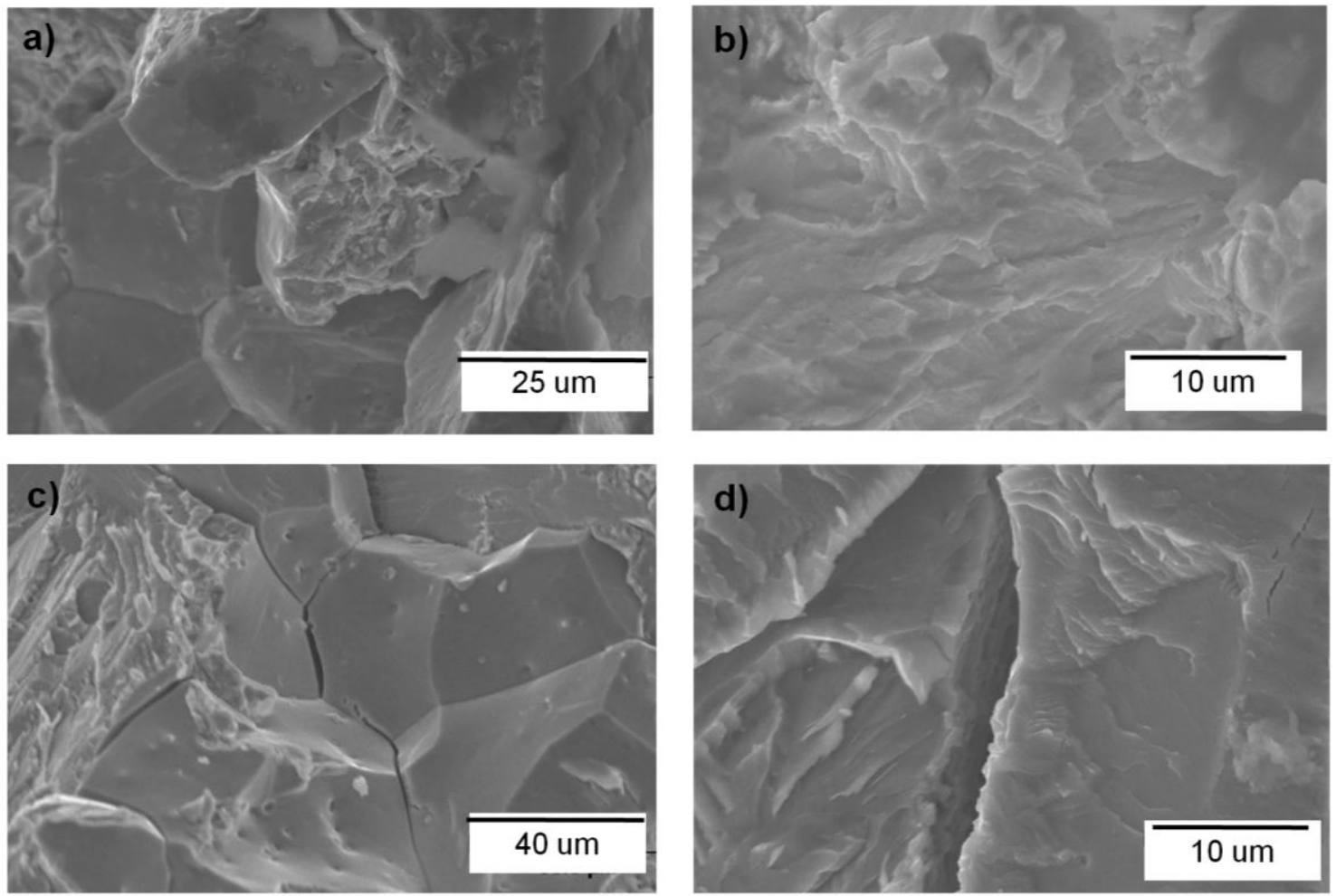

Figure 4.7: Fracture surfaces of $\operatorname{CoZr}(a, b)$ and CoTi (c,d) showing intergranular fracture and cleavage, respectively, for both materials. 
In summary, $\mathrm{MgR}$ intermetallics ( $\mathrm{MgCe}, \mathrm{MgNd}$, and $\mathrm{MgY}$ ) are demonstrated to not be among the recently discovered family of ductile B2 intermetallics, which includes $\mathrm{AgY}$ and $\mathrm{CuY}$. The group at Iowa State University/Ames Laboratory has also recently observed that $\mathrm{MgY}$ and $\mathrm{MgCe}$ tend to crack easily, indicating that they are brittle [35]. We have experimentally verified that the $\mathrm{MgR}$ intermetallics are, in fact, brittle and exhibit a cleavage-type fracture. Furthermore, even though AgY, CoTi and CoZr can exhibit significant tensile ductility, the fracture surfaces of these alloys do not show any signs of gross ductility (i.e. ductile dimples), as observed in $\beta$-brass (CuZn). Rather, the fracture surface indicates a mixed fracture mode (part transgranular cleavage and part intergranular). Two different EBSD-based methods are used to determine the crystallography of cleavage planes in the MgR compounds. In the case of MgY (analyzed by both methods), the results are similar, which gives confidence in the equivalence of both the methods. It is speculated that extrinsic factors, like testing environment, influence the selection of the cleavage plane.

\subsection{Acknowledgement}

This research was supported by the National Science Foundation, CAREER Award DMR 0547891. The authors thank Mr. James Wollmershauser for carrying out compression tests and Dr. Hongbin Bei (Oak Ridge National Laboratory) for providing fig 4.2f.

\subsection{References}

[1] K. Gschneidner, et al., Nature Materials 2(9) (2003), 587.

[2] A.M. Russell, et al., Acta Metall. Mater. 52(2004), 4033.

[3] K.H. Hahn, K. Vedula, Scripta Materialia 23, (1989), 7. 
[4] C.L. Fu, M.H. Yoo, Acta Metall. Mater. 40, (1992), 703.

[5] T.B. Massalski, Binary Alloy Phase Diagrams, 2nd Ed., vol.3, Institute of Materials, Minerals \& Mining, 2003.

[6] R.P. Mulay, et al., Acta Materialia, 58(7), (2010), 2788-2796

[7] R.P. Mulay, J.A. Wollmershauser, and S.R. Agnew, Magnesium Technology 2009, (TMS-AIME: Warrendale, PA: 2009) , 283

[8] P. Nagpal, I. Baker, Materials Characterization 27 (1991), 167.

[9] Personal Communication with Dr. Hongbin Bei.

[10] V. Randle, Microtexture Determination and its applications, 2nd Ed., ASM International, 1990.

[11] V. Sinha, M. Mills, J. Williams, Journal of Materials Science 42(19) (2007), 8334.

[12] Y.J. Ro, S.R. Agnew, R.P. Gangloff, Scripta Materialia 52, (2005), 531.

[13] G. Themelis, S. Chikwembani, J. Weertman, Materials Characterization 24, (1990), 27.

[14] S.F. Pugh, Phil. Mag. 45 (1954), 823.

[15] M. H. Yoo and C. L. Fu, Materials Science and Engineering: A 153(1-2)(1992), 470-478.

[16] H. Fu,et al., Computational Materials Science 44(2) (2008), 774-778.

[17] G. H. Cao, et al., Acta Materialia 58(12) (2010), 4298-4304.

[18] D. Shi, et al., Journal of Solid State Chemistry 182(10) (2009), 2664-2669.

[19] M. Lai, et al., Journal of Shanghai Jiaotong University (Science) 16(2) (2011),227230.

[20] T. H. Courtney, Mechanical Behavior of Materials, McGraw-Hill, 1990:383. 
[21] D.G. Pettifor, Mater. Sci. Tech. 8 (1992), 345.

[22] K. Chen, et al., Appl. Phys. 36 (2003), 2725.

[23] Y. Wu, W. Hu, European Physical Journal B 60(1) (2007), 75.

[24] X.M. Tao, et al. Solid State Communications 148(7-8) (2008), 314.

[25] J. R. Rice and R. Thomson, Philosophical magazine 29(1) (1974), 73-97.

[26] K.M. Chang, R. Darolia, H.A. Lipsitt, Acta Metall. Mater. 40 (1992), 2727.

[27] R. Darolia, K.M. Chang and J.E. Hack, Intermetallics. 1 (1993), 65-78.

[28] M.M. Shea and N.S. Stoloff, Materials Science \&Engineering 12, (1973), 245.

[29] W.K. Blanchard and L.A. Heldt, Metall. Trans. A 20A, (1989), 1439.

[30] P. Specht and P. Neumann Intermetallics 3(5) (1995), 365-368.

[31] P. Specht, M. Brede and P. Neumann, Fracture Toughness, fracture planes and BDT in stoichiometric FeAl and NiAl single crystals, in: Horton JA, et al. (Eds.). HighTemperature Ordered Intermetallic Alloys VI. Pittsburgh, PA: MRS (Materials Research Society); 1994.p. 207-212.

[32] D. Wu and I. Baker, Intermetallics 9(1) (2001), 57-65.

[33] L. M. Pike and C. T. Liu, Scripta Materialia 38(10) (1998), 1475-1480.

[34] Y. Liu, et al., Acta Metallurgica 37(2) (1989), 507-517.

[35] B. Stumphy, et al., Journal of Alloys and Compounds 460(1-2) (2008), 363. 


\title{
5. In situ neutron diffraction study of the Bauschinger
}

\section{effect in B2 structured CoZr ${ }^{i}$}

\author{
R.P. Mulay ${ }^{1}$, B. Clausen ${ }^{2}$, and S.R. Agnew ${ }^{1}$ \\ ${ }^{1}$ Materials Science and Engineering, University of Virginia, \\ Charlottesville, VA 22904-4745 \\ ${ }^{2}$ Los Alamos National Laboratory, LANSCE LC, Los Alamos, NM 87545 USA
}

\subsection{Abstract}

A combination of in situ neutron diffraction and elastoplastic self consistent (EPSC) modeling have been used to elucidate the role played by intergranular stresses in the Bauschinger effect in $\mathrm{B} 2$ structured $\mathrm{CoZr}$ at room temperature and $150^{\circ} \mathrm{C}$. It is shown that, when insufficient slip modes are present to accommodate arbitrary strains, the large intergranular stresses built up due to inhomogeneous plastic deformation are responsible for the observed Bauschinger effect. Upon the onset of secondary deformation mechanism(s), the stresses are more uniformly distributed among the grains and the influence of intergranular stresses on the Bauschinger effect diminishes. On the other hand, it is speculated that the contribution of intragranular (dislocation-based) stresses is responsible for the persistent Bauschinger effect past the transition point. Similar results are obtained at both room temperature and $150^{\circ} \mathrm{C}$ and, while the yield strength decreases with temperature, the high temperature stress-strain curve progressively becomes harder

\footnotetext{
${ }^{i}$ R. P. Mulay, B. Clausen and S.R. Agnew, In- situ neutron diffraction study of the Bauschinger effect in B2 structured CoZr, Metallurgical and Materials Transactions A, 2011; 42A(1):60-70. With kind permission from Springer Science+Business Media
} 
than the room temperature one. In light of this, the previously characterized yield strength anomaly in CoZr has been reexamined.

Keywords: diffraction, back stress, residual stress, intermetallic, crystal plasticity

\subsection{Introduction}

The Bauschinger effect, first reported in 1886 [1], is primarily concerned with an observed reduction in yield strength during reverse loading following prestraining. Its origin was initially attributed to residual stresses due to inhomogeneous deformation of individual grains in polycrystalline materials [2-4]. However, observations of the Bauschinger effect in single crystals provided evidence for dislocation-based explanations [5-6]. It was proposed that back-stresses created by dislocation pile-ups aid dislocation motion in the reverse direction [7]. Orowan [8] suggested an alternative dislocation-based theory, centered on a directional resistance to dislocation motion. Experiments were carried out to test these theories [9-11] and features of both dislocation-based theories were observed. Later research has revealed that dislocationbased internal stresses are also present in dislocation wall and cell structures, which are formed due to heterogeneous distribution of dislocations, and have been shown to be present under applied stress as well as in the unloaded states [12-15]. In spite of the extensive research carried out, the cause of the Bauschinger effect is still debated, more than 100 years after its discovery. By examining and modeling the internal stress

evolution directly, the contributions of intergranular stresses and dislocation-based mechanisms can be parsed. 
Recent research on the anomalous ductility of select B2-structured intermetallic compounds, including CoZr, reveals them to be ideal model materials for this study of the Bauschinger effect. Wollmershauser et al. [16] reported a transition in the plastic deformation at approximately $-250 \mathrm{MPa}$ for $\mathrm{CoZr}$ tested in compression at room temperature (Fig.5.1). They showed that, prior to this transition, only the $\langle 001>\{110\}$ slip mode is active, providing 3 independent slip systems. Beyond the transition, new deformation mechanisms are activated, which permit the grains to undergo arbitrary strains, as would 5 independent slip systems. However, there is some ambiguity regarding precisely which new deformation mechanism is activated (polycrystal plasticity modeling showed that either $<111>\{1 \overline{1} 0\}$ or $<110>\{1 \overline{1} 0\}$ type slip can capture the experimental observations [16].) The intergranular stresses before and after this transition point are markedly different and, therefore, in-situ cyclic loading experiments were performed within both regimes. In this way, the role of intergranular stresses in the Bauschinger effect may be examined under conditions where they are large and small, before and after the transition, respectively. 


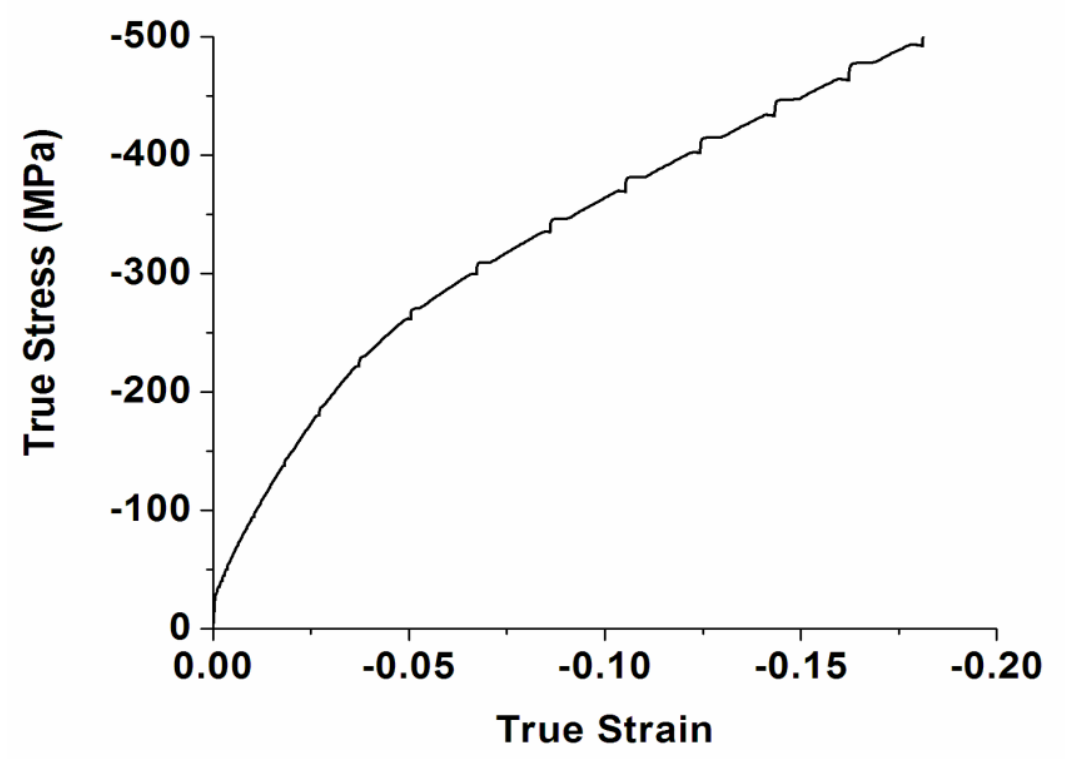

Figure 5.1: Compressive stress-strain curve of CoZr at room temperature [16]. The test has been stopped intermittently and held at a constant load to collect neutron diffraction patterns, giving rise to the peaks apparent at higher stresses. Note the transition in plastic deformation at $250 \mathrm{MPa}$.

The objective of this research is to characterize and understand the role of intergranular stresses in the strong Bauschinger effect observed in the B2 structured compound, CoZr. In situ neutron diffraction is used to measure the internal elastic strains, and elastoplastic self-consistent (EPSC) modeling (which only incorporates the effect of intergranular stresses and not any dislocation-based back-stress mechanisms) is used to simulate the macroscopic Bauschinger effect and the attendant internal strain evolution. 


\subsection{Experimental procedure}

\subsubsection{Alloy preparation and Characterization}

Cobalt (99.9 wt\%) and zirconium (99.5 wt \%) obtained from Alfa Aesar were arc melted and drop cast as a cylinder (24.5 mm diameter) in an Ar atmosphere at the Oak Ridge National Laboratory (ORNL). The major impurities present in the raw materials are provided in Table 5.1. Since the mass loss was below $0.06 \%$, additional chemical analysis was not performed. The drop cast cylinder was homogenized in a horizontal tube vacuum furnace for 24 hours at $1000{ }^{\circ} \mathrm{C}$ and a pressure of $\sim 10^{-3} \mathrm{~Pa}$. The cleaned cylinder was then sealed in an evacuated stainless steel container and preheated to $1000^{\circ} \mathrm{C}$ for 1 hour prior to extrusion. Extrusion was also performed at ORNL with a 4:1 reduction ratio, resulting in a final sample diameter of $\sim 12.7 \mathrm{~mm}$. The extruded billet was then turned on a lathe to remove the cladding, expose the alloy, and then, further turned down to a diameter of $10 \mathrm{~mm}$. Cylindrical compression specimens were then sectioned $(20 \mathrm{~mm}$ length) using electro-discharge machining (EDM). Finally, the sectioned specimens were individually encapsulated in a quartz ampoule, with titanium as a getter, back filled with Ar, and annealed at $1000{ }^{\circ} \mathrm{C}$ for 1 hour as per established practices [17]. A suitable etchant could not be found and, hence, the grain size was characterized using electron backscattered diffraction (EBSD). To produce a surface suitable for EBSD, the sample was electro polished in an electrolyte composed of $85 \%$ methanol and $15 \%$ sulphuric 
Table 5.1: Major impurities in raw materials as provided by supplier.

\begin{tabular}{|l|c|c|c|c|c|c|c|c|c|c|c|}
\cline { 2 - 11 } \multicolumn{1}{c|}{} & \multicolumn{9}{c|}{ Impurities (ppm) } \\
\hline Element & Al & C & Cr & Fe & Hf & N & Nb & Ni & 0 & Ta & W \\
\hline Cobalt & $<4$ & 6 & $*$ & $<3$ & $*$ & $<1$ & $*$ & 148 & 18 & $*$ & $*$ \\
\hline Zirconium & $<30$ & $<21$ & $<50$ & $<85$ & 80 & $<20$ & 50 & $<35$ & $<70$ & $<100$ & $<50$ \\
& & & & & & & & & & & \\
\hline
\end{tabular}

* Not reported

acid. The grain size was determined using the linear intercept method and found to be approximately $24 \mu \mathrm{m}$. Because it is an important input for the polycrystal plasticity modeling, the initial texture was measured using the High-Pressure Preferred Orientation diffractometer (HIPPO), which allows for rapid characterization of full orientation distributions of a bulk sample (i.e. it is not surface limited like x-ray or electron-based techniques) [18].

\subsubsection{Compression tests and in-situ neutron diffraction}

The cyclic compression tests were performed on a custom built Instron load frame in the flight path of the time of flight neutron beam line, Spectrometer for Materials Research at Temperature and Stress (SMARTS), at the Manuel Lujan Jr. Neutron Scattering Center, LANSCE. A detailed description of SMARTS is given in previous publications [19] and only a short description is provided here. The loading axis is oriented at $45^{\circ}$ relative to the incident beam and two detector banks are situated at $\pm 90^{\circ}$ making it possible to simultaneously collect two complete diffraction patterns one parallel and one perpendicular to the applied load. Due to the horizontal in-situ testing 
rig, the compression sample was preloaded to a stress of $-5 \mathrm{MPa}$ to hold it in place. The initial $d_{h k l}^{o}$ spacing measured at $-5 \mathrm{MPa}$ is considered to be the "stress free" spacing.

Cyclic compression tests were carried out in-situ, within a neutron beam, at room temperature and $150{ }^{\circ} \mathrm{C}$. An extensometer was attached to the sample to measure the strain. The samples were lubricated using molybdenum disulfide $\left(\mathrm{MoS}_{2}\right)$ grease. The diameter of the samples measured after the compression tests showed a very small variation over the length of the sample $( \pm 0.01 \mathrm{~mm})$, indicating that no barreling took place. The tests were carried out in load control at a loading rate of $50 \mathrm{MPa} / \mathrm{min}$. Five cyclic loads and unloads were carried out in the stress range of 100-500 $\mathrm{MPa}$ so as to capture the Bauschinger effect both before and after the aforementioned transition in plastic deformation. For the $150{ }^{\circ} \mathrm{C}$ test, an induction furnace was used to heat the sample in air. Thermocouples were attached to the sample to ensure that a constant temperature was maintained throughout the test.

The neutron diffraction measurements were carried out at stress intervals of 25 or $50 \mathrm{MPa}$. The incident beam apertures were controlled to give a beam size of $\sim 5 \mathrm{~mm}^{2}$. The load frame was held at a constant load during diffraction pattern collection and the hold time was varied to obtain a constant neutron count at the detectors (governed by the source current and the material neutron absorption and scattering cross sections). For this material, the collection time is approximately 10-12 minutes per pattern. Internal strains within grains that satisfy the Bragg condition for a given (hkl) reflections are calculated from the difference between the measured and 'stress-free' lattice spacing:

$$
\varepsilon_{h k l}=\frac{d_{h k l}-d_{h k l}^{o}}{d_{h k l}^{o}}
$$


where $d_{h k l}$ is the measured lattice spacing and $d_{h k l}^{o}$ is the 'stress-free' lattice spacing. The peak shifts and subsequent lattice strains were calculated using single peak fits with an instrument specific code developed at LANSCE [20] which uses General Structural Analysis System (GSAS) [21].

\subsubsection{EPSC modeling}

The EPSC modeling approach has been described in detail in previous publications [22] and only a short description is provided here. The model is based on the Eshelby solution of the inclusion problem [23] where each grain is modeled as an ellipsoidal inclusion embedded in a homogeneous effective medium with anisotropic properties characteristic of the textured polycrystalline aggregate. In this case, a set of approximately 2900 grain orientations were volume weighted to best represent the experimentally measured initial texture. Self-consistency refers to the requirement that the volume weighted average of the stresses and strains experienced by the individual grains must equal the externally applied macroscopic boundary conditions. This approach captures the fact that grains in hard orientations may remain elastic well past the macroscopic proportional limit yield stress whereas grains in softer orientations carry a smaller portion of the elastic stresses, since they accommodate the imposed strain by plastic deformation. The EPSC approach explicitly allows for the variations in stress and strain levels from one grain to another, which allows for direct comparison between simulated and experimental in-situ diffraction results. Again, the model captures the effects of intergranular stresses but does not incorporate the effect of dislocation-based back-stresses. 
The known single crystal elastic constants are used as inputs for the model. The fitting parameters of these models are the critical resolved shear stress (CRSS) and hardening values which describe the individual deformation modes. The hardening response is described by the parameters of an empirical Voce hardening rule:

$$
\tau=\tau_{0}+\left(\tau_{1}+\theta_{1} \Gamma\right)\left\{1-\exp \left(\frac{-\theta_{0} \Gamma}{\tau_{1}}\right)\right\}
$$

where $\tau$ is the instantaneous CRSS; $\tau_{0}$ and $\tau_{0}+\tau_{1}$ are the initial and back-extrapolated CRSS, respectively; $\theta_{0}$ and $\theta_{1}$ are the initial and final asymptotic hardening rates, respectively; and $\Gamma$ is the accumulated plastic shear in the grain. An "inverse approach" is used, where the stress-strain curve predicted by the model is fitted to the experimental curve to obtain the values for CRSS and hardening parameters. In many cases (see table 5.3 and 5.4), the behavior was such that a simpler, linear hardening rule was sufficient to capture the observation, thus reducing equation 2 to a simpler one with only 2 fitting parameters,

$$
\tau=\tau_{0}+\theta_{1} \Gamma
$$

The slip systems seen to be active in B2 compounds are $<001>\{110\}$ and $<111>\{1 \overline{10}\}$ or $<110>\{1 \overline{10}\}[24]$. It is noted that the experimental stress-strain curve alone provides insufficient data to uniquely determine the 2 to 4 hardening parameters required to describe each slip system. However, by comparing the development of internal elastic strains predicted by the model to the experimentally measured internal elastic strains it is possible to rule out some possibilities. The latent hardening of each slip system $a$ by other slip systems $\beta$ of the same (or other) slip modes is incorporated using a hardening matrix, 
h $[25,26]$. The latent hardening parameters were not adjusted; in the present study we assumed all slip systems to contribute equally to the hardening of all others, i.e. the components $h_{\alpha \beta}=1$.

When the relation between the stress rate and strain rate is non-linear, it is usual to assume a linearized expression so that the equilibrium equation can be solved within self consistent formulations $[27,28]$. The interaction equation relates the total strain rate in a grain to that in the surrounding medium;

$$
\left(\dot{\boldsymbol{\sigma}}_{C}-\boldsymbol{\sigma}\right)=-\beta_{e f f} \mathbf{L}^{*}:\left(\dot{\boldsymbol{\varepsilon}}_{C}-\dot{\boldsymbol{\varepsilon}}\right)
$$

where $\boldsymbol{\sigma}_{C}$ and $\boldsymbol{\sigma}$ are the stress rate in the grain and in the medium respectively, $\boldsymbol{\varepsilon}_{C}$ and $\boldsymbol{\varepsilon}$ are the total strain rate in the grain and in the medium respectively; $\mathbf{L}^{*}$ is the effective stiffness and $\beta_{\text {eff }}$ is a parameter which controls the strength of coupling between the stress-rate deviations and the associated strain-rate deviations between the grain and the bulk medium.

The value of $\beta_{\text {eff }}$ is normally set to 1 , in respect of the solution to the fully-elastic problem [23]. However, it may be varied to alter the type of interaction. For example, setting $\beta_{\text {eff }}=0$ would be similar to Sach's assumption, yielding an infinitely compliant interaction where every grain would adopt the stress-rate of the aggregate. In the present context, such an assumption would make no sense, since we directly measure differences in the internal strain levels of different grains, using neutron diffraction. At the other extreme, an infinitely stiff interaction, $\beta_{\text {eff }}=\infty$, is at the heart of Taylor's assumption, which permits wild variations in the stress-rates of individual grains, while forbidding any differences in the strain rates. In this study, the value of $\beta_{\text {eff }}$ was obtained by fitting 
the model to the experimentally observed cyclic loads and unloads. A similar approach was taken previously, in the context of viscoplastic self-consistent modeling (VPSC) [29, 30].

\subsection{Results}

\subsubsection{Stress Strain curves}

The stress strain curves obtained at room temperature and $150{ }^{\circ} \mathrm{C}$ are shown in Figure 5.2. The load plateau regions correspond to the points where neutron diffraction data were collected at a constant load. In other words, the samples crept slightly during the collection of the neutron diffraction data. It can be clearly seen that although the sample tested at $150^{\circ} \mathrm{C}$ yields at a lower value, the curve progressively becomes harder than the room temperature curve past the yield point, i.e. it strain hardens more rapidly and to a greater extent. The room temperature sample yields at $-50 \mathrm{MPa}$ (defined by a $0.2 \%$ offset) while $150^{\circ} \mathrm{C}$ sample yields at $-45 \mathrm{MPa}$. Since the initial strain hardening is almost linear, a transition point in the strain hardening behavior can be defined using a $1 \%$ plastic strain offset similar to a yield point. While defining the transition point corresponding to the $1 \%$ plastic strain offset, the slope of the initial linear strain hardening is used akin to using the elastic modulus while defining the yield point. The transition is observed to occur at $-250 \mathrm{MPa}$ at room temperature and $-270 \mathrm{MPa}$ at 150 ${ }^{\circ} \mathrm{C}$. Thus, the aforementioned plastic transition occurs at a higher value at $150{ }^{\circ} \mathrm{C}$ than it

does at room temperature. Other than these fine points, the overall response of the material at the two temperatures is remarkably similar. 


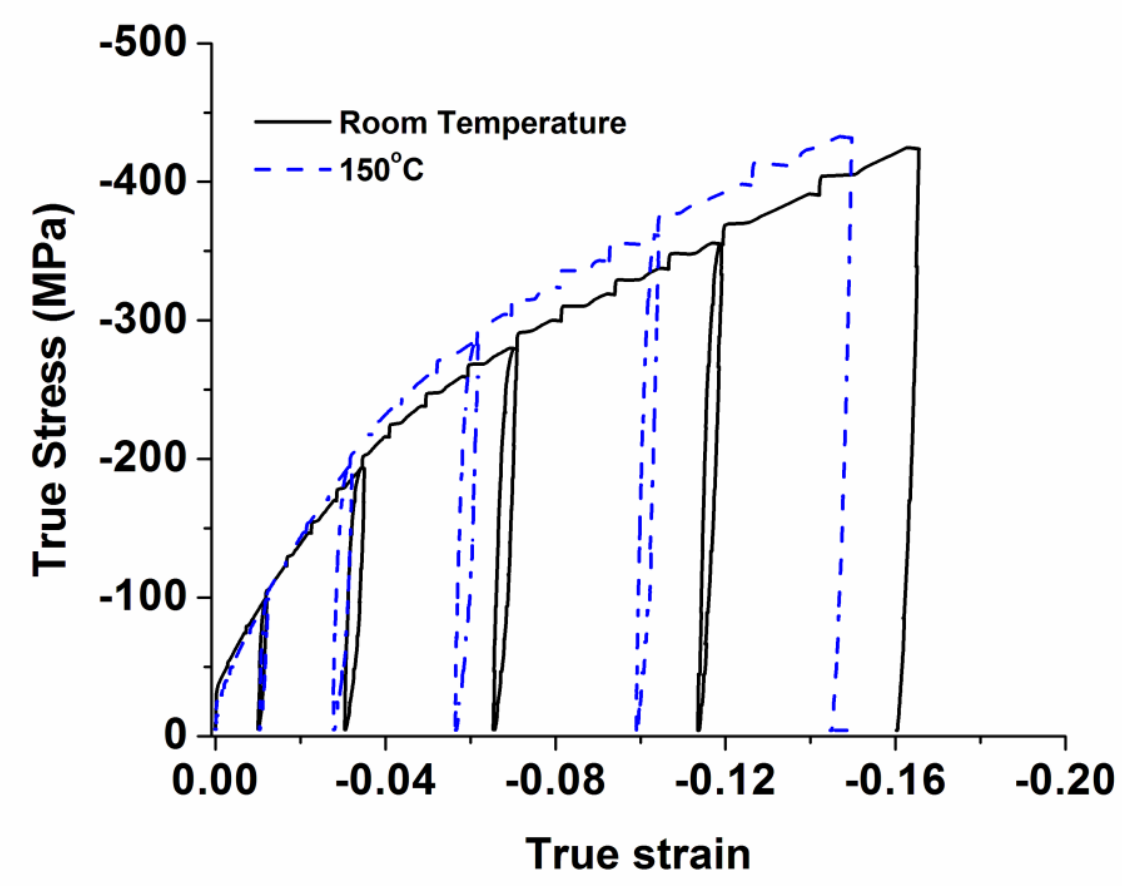

Figure 5.2: In-situ stress strain curves, including unload-reload cycles, at room temperature and $150^{\circ} \mathrm{C}$.

On unloading, the material begins to yield in the reverse direction well before zero load is reached. Observation of this strong Bauschinger phenomenon was one of the reasons for initiating this study in the first place. Many different parameters have been used to evaluate the magnitude of the Bauschinger effect [31]. Parameters that can be determined from this study are the magnitude of yield lowering and the area of hysteresis loop formed due to cyclic loading. A gradual change from elastic to plastic deformation, as seen in a stress-strain curve, leads to some uncertainty in determining the actual magnitude of yield lowering [31]. Hence, the "loop-size" parameter, which refers to the area of the hysteresis loop normalized by the peak stress (the height of the particular loop being considered), was chosen in this study as an evaluation of the magnitude of the 
Bauschinger effect. At both temperatures, it can be clearly seen that the "loop size" increases between the yield and transition points and starts to decrease past the transition point; the Bauschinger effect is most prominent between the yield and transition points and then it diminishes beyond the transition (Fig.5.3). Figure 5.3 provides further evidence that the two experimental temperatures yield a very similar behavior.

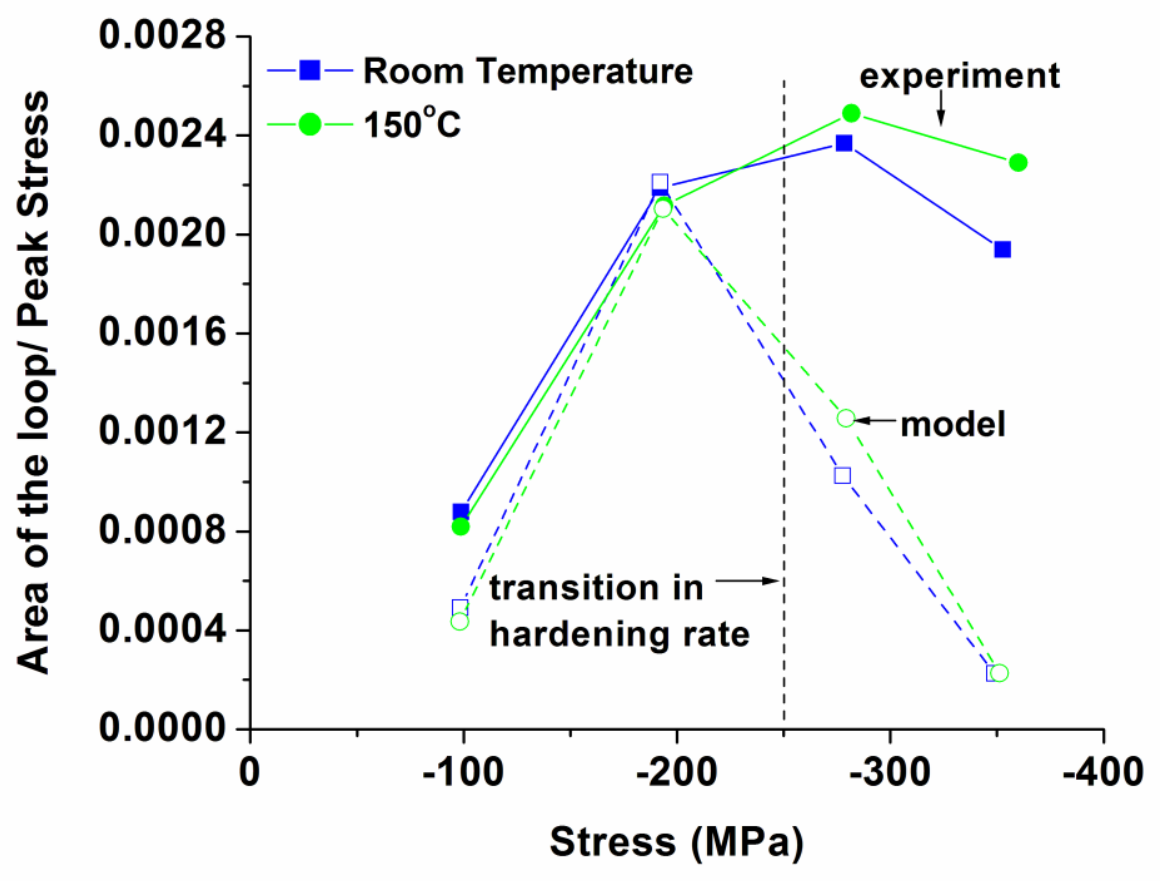

Figure 5.3: A plot of the "loop size" parameter as a function of stress for both the experimental and modeled stress-strain curves at room temperature and $150^{\circ} \mathrm{C}$.

\subsubsection{Neutron diffraction and internal strains}

The diffraction patterns collected in the "stress free" state at room temperature and $150^{\circ} \mathrm{C}$ are shown in Figures 5.4a and 5.4b, respectively. Even though CoZr is a line compound, no peaks corresponding to other phases are seen, indicating that the samples are single phase. A few additional peaks are seen in the pattern collected at $150^{\circ} \mathrm{C}$, but 
the peaks correspond to the thermocouple wire used to measure the temperature. Since these peaks do not overlap with the CoZr peaks, their presence can be considered insignificant. Heating to $150^{\circ} \mathrm{C}$ in air does not result in the presence of any additional peaks.

a)

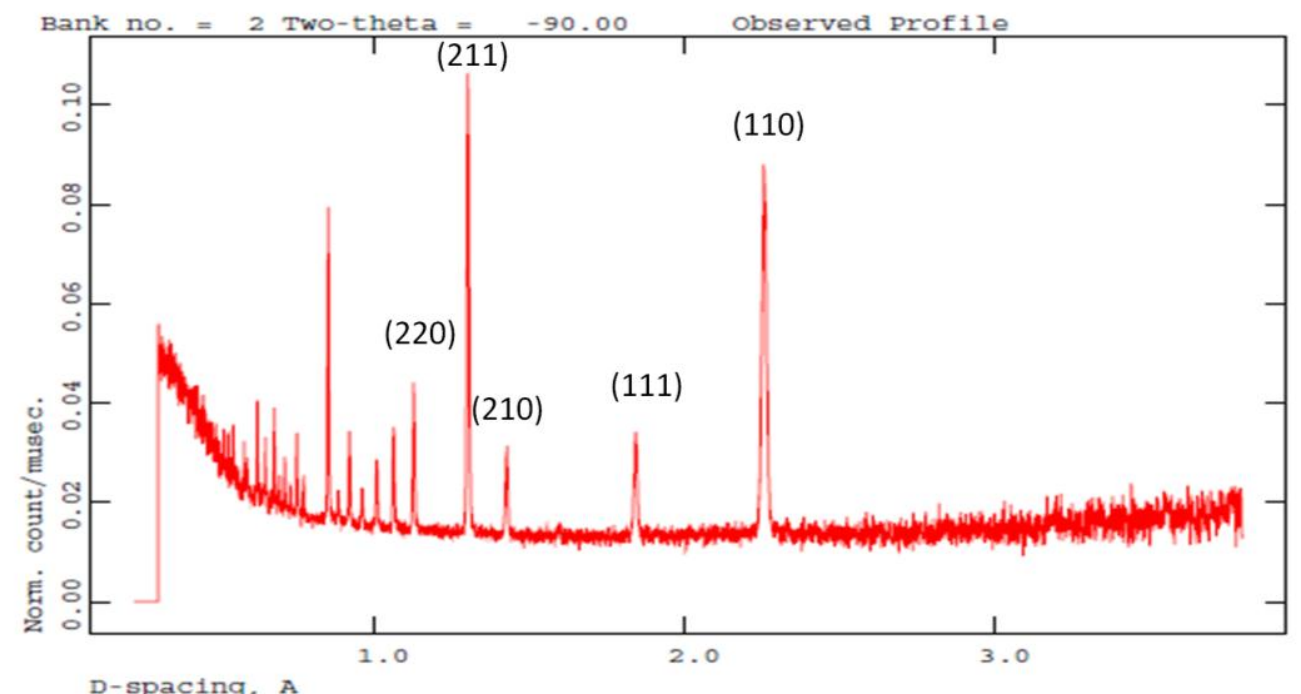

b)

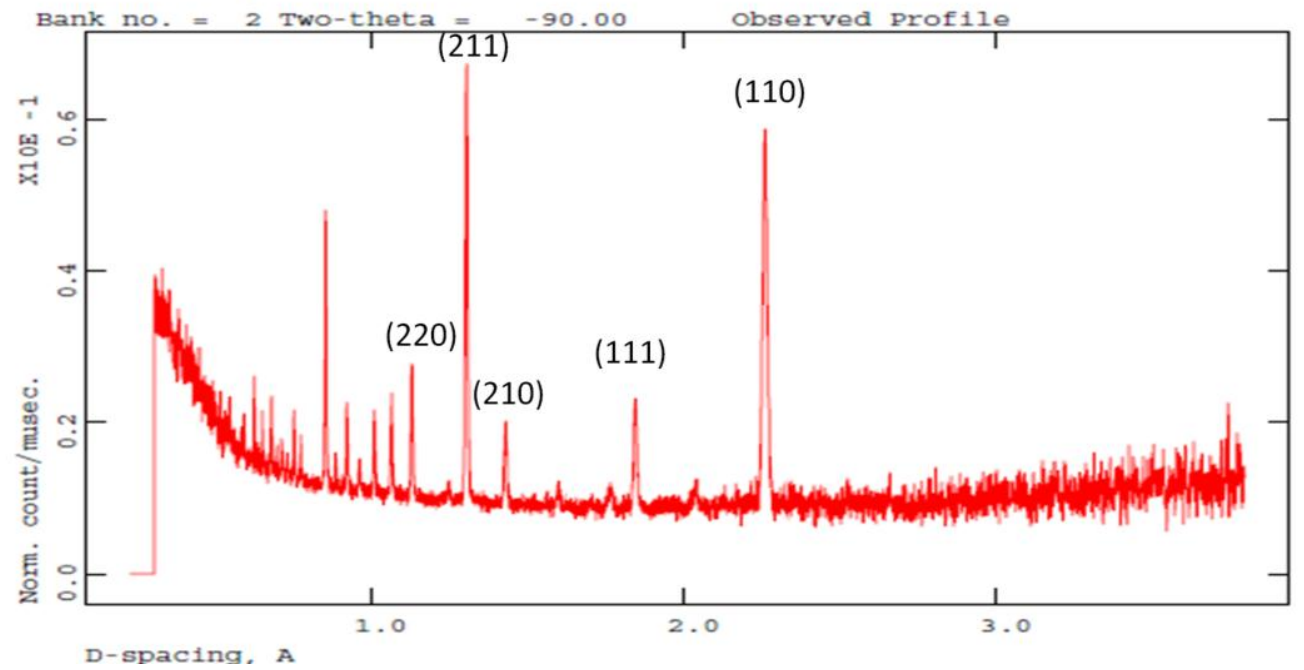

Figure 5.4: Diffraction patterns of CoZr obtained in the "stress-free" state parallel to the loading direction at, a) room temperature and b) $150^{\circ} \mathrm{C}$. 
Since the samples were tested in a highly annealed condition; since cubic crystals have no anisotropy in the coefficient of thermal expansion; and since the elastic parameters of $\mathrm{CoZr}$ are isotropic $(\mathrm{A}=1[32])$, it is reasonable to assume that there is very little intergranular stress inherited from previous processing. Figures 5.5 and 5.6 show the internal elastic strains observed during the five cyclic loads and unloads at room temperature and $150^{\circ} \mathrm{C}$, respectively. The plots contain data collected for the (111) and (210) diffraction peaks. The uncertainty in determining the internal strains is related to the uncertainty in determining the peak positions. This depends on the diffraction peak being analyzed. For the (111) and (210) peaks, the uncertainty ranges from 30-50 microstrain. The solid line corresponds to the expected average intergranular strain given by Hooke's law, i.e., its slope is the elastic modulus. The elastic modulus was calculated from the experimental stress-strain curves (Fig.5.2) and is found to be $120 \mathrm{GPa}$ at both room temperature and $150^{\circ} \mathrm{C}$. Other peaks were also examined, but for clarity only these two are presented. These two well illustrate the range of behaviors, though other peaks, e.g. (100) or (200), are predicted to have even more extreme behavior they were weaker and, therefore, contained more experimental scatter. It is important to emphasize that bulk neutron diffraction experiments, like those presently reported, do not reveal the internal stresses within individual grains. Rather, they probe sets of grains whose $\{\mathrm{hkl}\}$ plane normal vectors are parallel to the loading direction. In short, different subsets of grains are polled by the different $\{\mathrm{hkl}\}$ diffraction peaks according to their satisfaction of Bragg's law. Prior to macroscopic yielding (seen clearly in Figs. 5.5a and 5.6a), it can be seen that the internal elastic strains in the differently oriented grains develop linearly and diverge only slightly since this material has very low single crystal elastic anisotropy. 

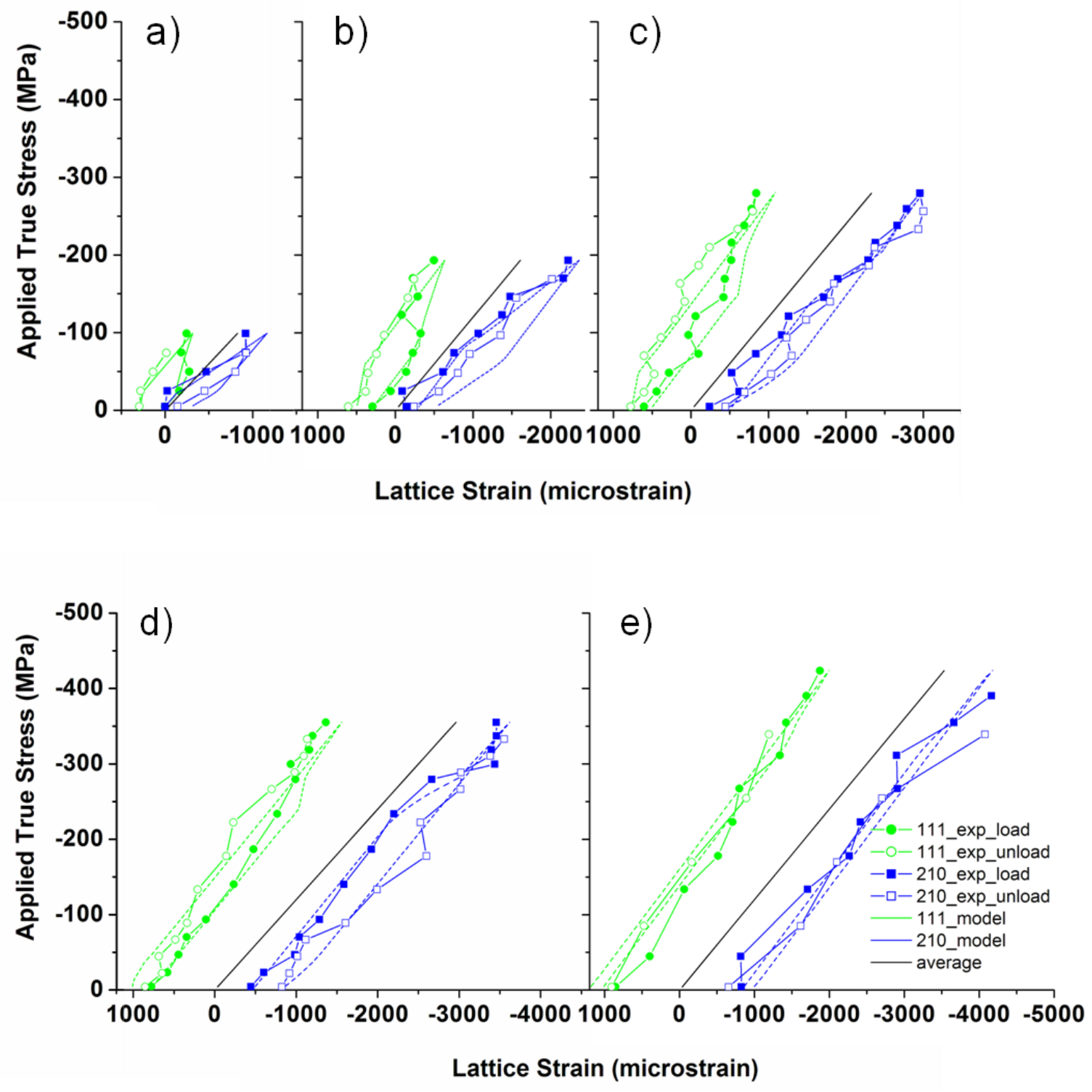

Figure 5.5: Experimentally measured and simulated internal elastic strains (parallel to the loading direction) for the (111) and (210) sets of CoZr grains at room temperature for the five different loads and unloads, respectively.

Upon macroscopic yielding, the (210) oriented grains start accumulating internal elastic strain at a much faster rate, while the (111) oriented grains diminish their share of 
the internal elastic strains. The strong variation exhibited by these two subsets of grains is indicative of the strong plastic anisotropy of the individual crystals which compose the aggregate. The (210) grains are oriented in a hard direction for slip and, hence, they accommodate more strain elastically. In contrast, the (111) grains are oriented in a soft direction and, upon macroscopic yielding, they start accommodating more strain plastically and start diminishing their share of elastic strains. On unloading (Figs. 5.5 and 5.6), the compressive internal strains in the differently oriented grains start reducing at approximately the same rate, again due to the relative elastic isotropy. However, before zero stress in reached there is a change in the rate of decrease of internal strains; see the unload curves carried out prior to the transition. The internal strains in the hard (210) grains start decreasing at a much faster rate while the internal strains in the soft (111) grains start decreasing at a much slower rate (Fig. 5.5a). This indicates the hard grains (which are now expanding rapidly, due to the decreasing compressive load) are preventing the softer grains from expanding, and this is causing a buildup of tensile stresses in the soft grains causing them to yield in the reverse direction. It is to be noted that this phenomenon appears to decrease in the unloads performed past the transition point (Figs. 5.5e and 5.6e); this indicates that the role of intergranular stresses in the Bauschinger effect has reduced significantly, at this point.

It is important to note that the first two unloads (Figs. $5.5 \mathrm{a}, \mathrm{b}$ and $5.6 \mathrm{a}, \mathrm{b}$ ) are prior to the plastic transition (i.e., the transition in strain hardening behavior at $\sim-250$ $\mathrm{MPa}$ ), and hence, on loading only one change in the internal strains slope is seen. After 


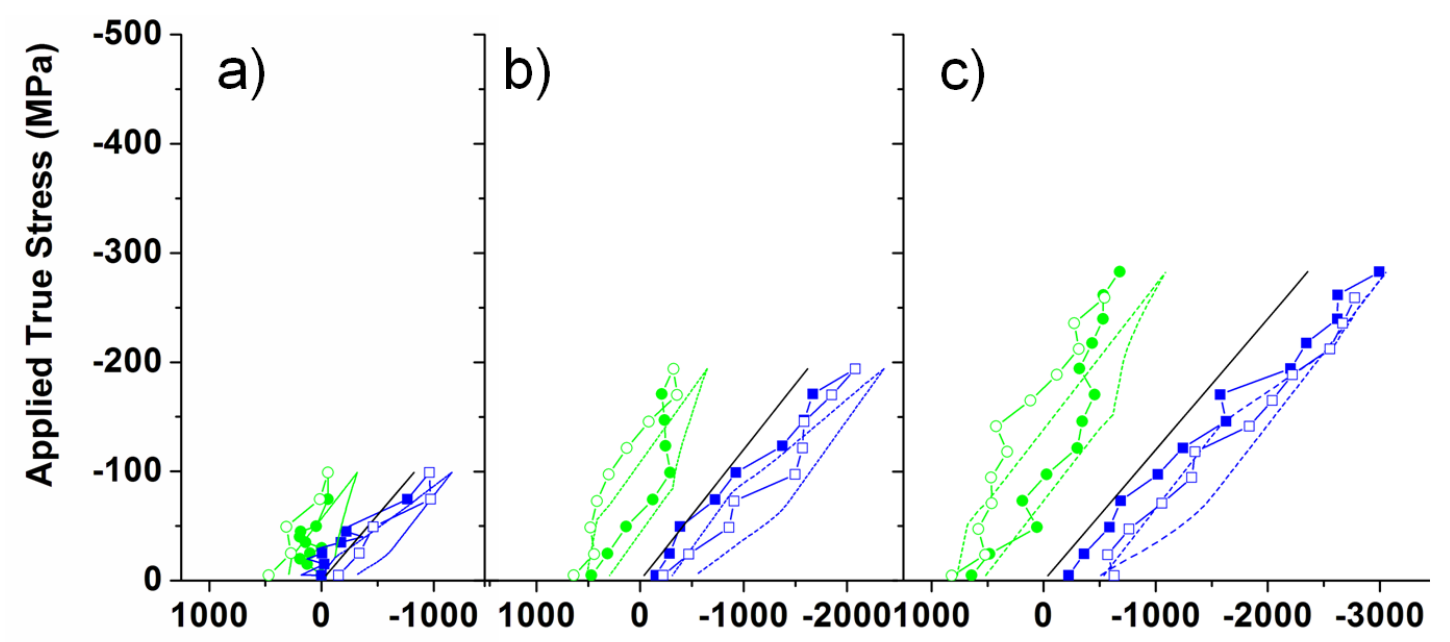

Lattice Strain (microstrain)

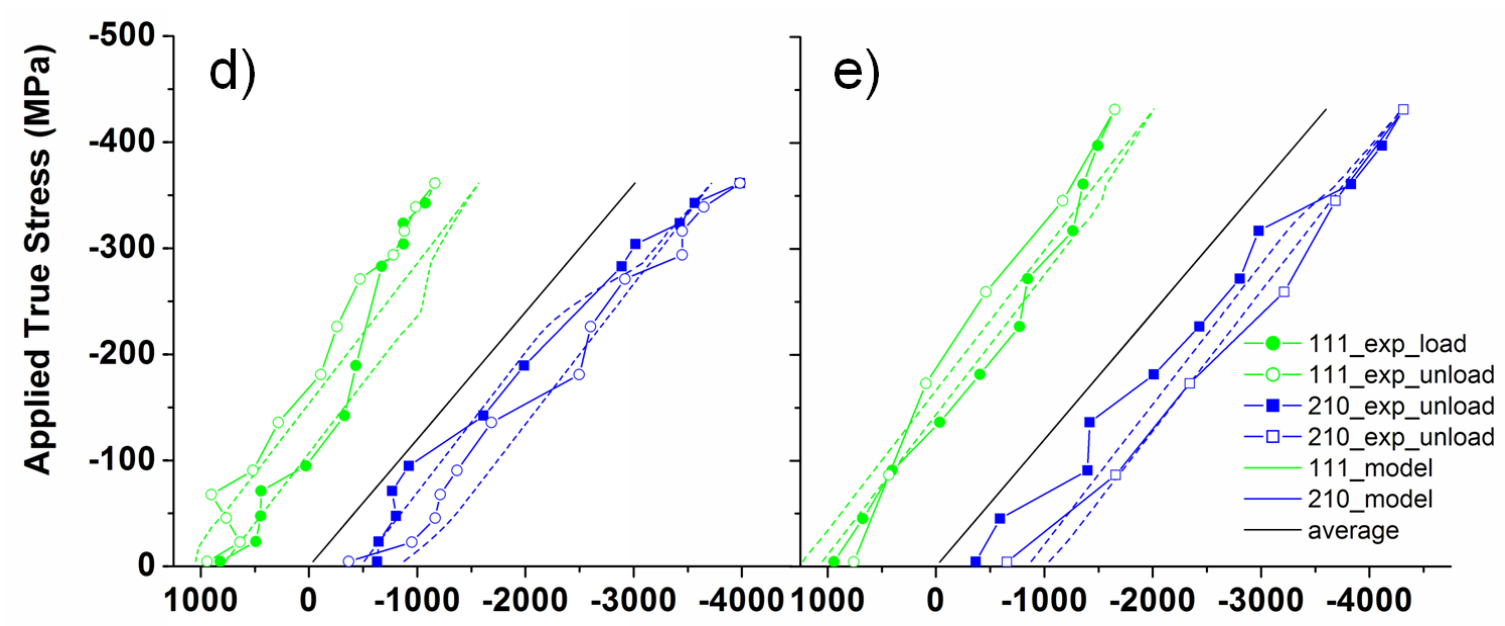

Lattice Strain (microstrain)

Figure 5.6: Experimentally measured and simulated internal elastic strains (parallel to the loading direction) for the (111) and (210) sets of $\mathrm{CoZr}$ grains at $150{ }^{\circ} \mathrm{C}$ for the five different loads and unloads, respectively .

the aforementioned plastic transition, it is expected that the "hard grains" will also accommodate strain plastically and the rate of increase in internal strains of both hard and 
soft grains will be equal again. This can be seen in the loading carried out past the transition. Again it can be seen that the overall behaviors at room temperature and $150^{\circ} \mathrm{C}$ are strikingly similar.

It is to be noted that the diffraction patterns showed no evidence of stress induced phase transformations. If a pseudoelastic phase transformation was occurring in a very small volume fraction (undetectable in the diffraction patterns), the Bauschinger effect would increase (rather than decrease) past the transition in strain hardening. This observation aids the argument that the ductility in $\mathrm{CoZr}$ is due to the activation of secondary slip modes and not a phase transformation.

\subsubsection{EPSC modeling}

The single crystal constants used in the model [32] are shown in Table 5.2. The EPSC model has been used to identify the operative slip systems of a variety of materials, ranging from hexagonal close packed $\mathrm{Mg}$ and $\mathrm{Be}$ alloys $[33,34]$, to $\mathrm{B} 2$ compounds $\mathrm{NiAl}$, $\mathrm{CuZn}$, and CeAg [35]. On similar lines, we have fitted the experimental stress- strain curves (Fig. 5.7 and 5.8) using the $<001>\{110\}$ slip system to capture the initial strain hardening followed by a late activation of a secondary mechanism (either $<111>\{1 \overline{10}\}$ or $<110>\{1 \overline{10}\})$. The Voce hardening parameters which best fit the observed stressstrain curves are given in Tables 5.3 and 5.4 for the room temperature and $150^{\circ} \mathrm{C}$ test respectively. It was again impossible to distinguish which of the two secondary modes better describe the behavior. In order to avoid redundant figures, only the results of $<110>\{110\}$ are shown, however, it is acknowledged that this is not a completely unique solution and similarly good results were obtained by using the $<111>\{110\}$ slip mode. 
Table 5.2: Single-crystal elastic constants [32] and anisotropy factor used as inputs for EPSC modeling of CoZr both at room temperature and $150{ }^{\circ} \mathrm{C}$.

\begin{tabular}{|c|c|c|c|}
\hline $\mathbf{C}_{\mathbf{1 1}}(\mathbf{G P a})$ & $\mathbf{C}_{\mathbf{1 2}}(\mathbf{G P a})$ & $\mathbf{C}_{\mathbf{4 4}}(\mathbf{G P a})$ & $\mathbf{A}$ \\
\hline 205 & 107 & 49 & 1 \\
\hline
\end{tabular}

Initially, the value of $\beta_{\text {eff }}$ (interaction stiffness between grain and matrix) was taken to be 1(see equation 4). However, the Bauschinger effect was over predicted and the "loop" size was larger than that observed experimentally for all unloads (see Fig. 5.7). Using $\beta_{\text {eff }}$ as a fitting parameter, a value of 0.82 was found to best describe the experimental results. Reducing this value further caused a continuous lowering of the predicted Bauschinger effect and, when the value of $\beta_{\text {eff }}$ was reduced to 0.72 , no Bauschinger effect was observed upon unload to zero stress.

Table 5.3: Predicted Voce hardening Parameters for the active slip modes in CoZr at room temperature

\begin{tabular}{|c|c|c|c|c|}
\hline & $\boldsymbol{\tau}_{\mathbf{0}}(\mathbf{M P a})$ & $\boldsymbol{\tau}_{\mathbf{1}}(\mathbf{M P a})$ & $\boldsymbol{\theta}_{\mathbf{0}}(\mathbf{M P a})$ & $\boldsymbol{\theta}_{\mathbf{1}}(\mathbf{M P a})$ \\
\hline$<100>\{011\}$ & $\mathbf{1 0}$ & $\mathbf{0 . 5}$ & $\mathbf{2 3 0}$ & $\mathbf{2 2 0}$ \\
\hline$<110>\{11 \overline{0}\}$ & $\mathbf{2 2 0}$ & $\mathbf{1 0}$ & $\mathbf{1 0 0}$ & $\mathbf{5 0}$ \\
\hline$<111>\{1 \overline{0}\}$ & $\mathbf{1 8 5}$ & $\mathbf{0}$ & $\mathbf{2 9 0}$ & $\mathbf{2 9 0}$ \\
\hline
\end{tabular}

Table 5.4: Predicted Voce hardening Parameters for the active slip modes in CoZr at $150^{\circ} \mathrm{C}$

\begin{tabular}{|c|c|c|c|c|}
\hline & $\boldsymbol{\tau}_{\mathbf{0}}(\mathbf{M P a})$ & $\boldsymbol{\tau}_{\mathbf{1}}(\mathbf{M P a})$ & $\boldsymbol{\theta}_{\mathbf{0}}(\mathbf{M P a})$ & $\boldsymbol{\theta}_{\mathbf{1}}(\mathbf{M P a})$ \\
\hline$<100>\{011\}$ & $\mathbf{1 0}$ & $\mathbf{0}$ & $\mathbf{2 5 5}$ & $\mathbf{2 5 5}$ \\
\hline$<110>\{1 \overline{10}\}$ & $\mathbf{2 3 0}$ & $\mathbf{0}$ & $\mathbf{1 1 0}$ & $\mathbf{1 1 0}$ \\
\hline$<111>\{110\}$ & $\mathbf{1 8 5}$ & $\mathbf{0}$ & $\mathbf{3 3 0}$ & $\mathbf{3 3 0}$ \\
\hline
\end{tabular}


a)

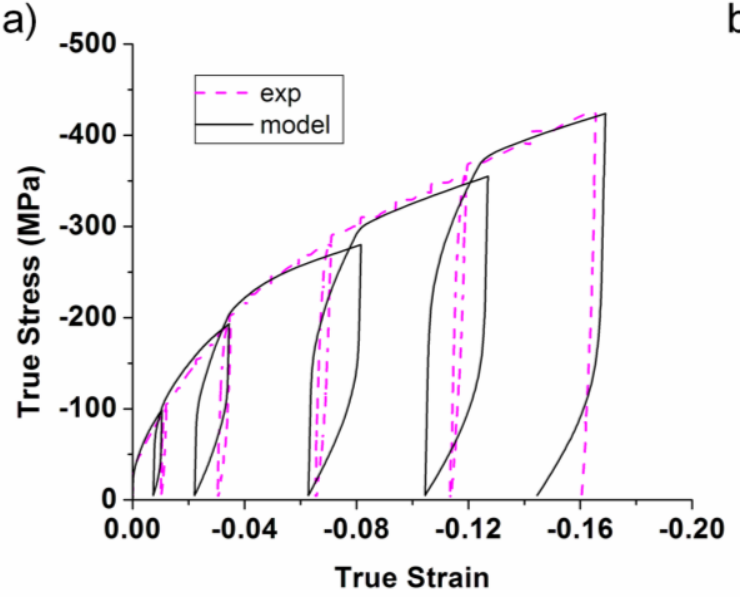

b)

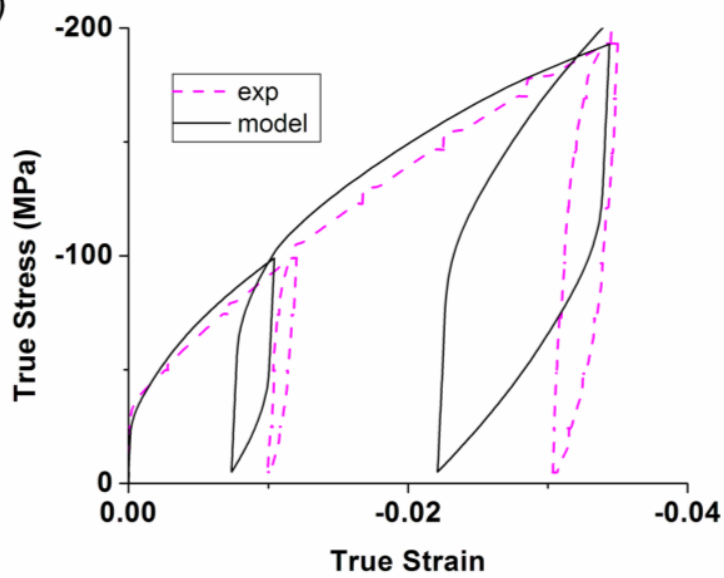

Figure 5.7: a) EPSC fits superimposed on the experimental stress strain curves for value of $\beta_{\text {eff }}=1$ at room temperature. b) The part of the stress strain curve prior to the transition point has been magnified for greater clarity.

Figure 5.8 shows the simulated stress-strain curve superimposed on the experimental stress-strain curve with the initial part of the curve magnified. It can be seen that the first two unloads predicted by the model agree well with the experiments in case of both the temperatures. The width of the first loop is slightly under predicted, but that of the second loop is almost perfectly predicted. The overall trend, i.e. the increase in the Bauschinger effect with increasing pre-strain prior to the transition point, is well-captured by the model (Figs.5.3 and 5.8). The remaining unloads (Figs. 5.8) are performed past the transition point and the width of these loops is under-predicted by the model. This can also be seen from the plot of the "loop-size parameter" (Fig.5.3). In other words, the decrease in the Bauschinger effect past the transition point is over predicted. It is important to note that, even though the EPSC model does not incorporate any dislocationbased back-stresses, it still reasonably predicts the macroscopic Bauschinger effects, by considering only the intergranular stresses. 
a)

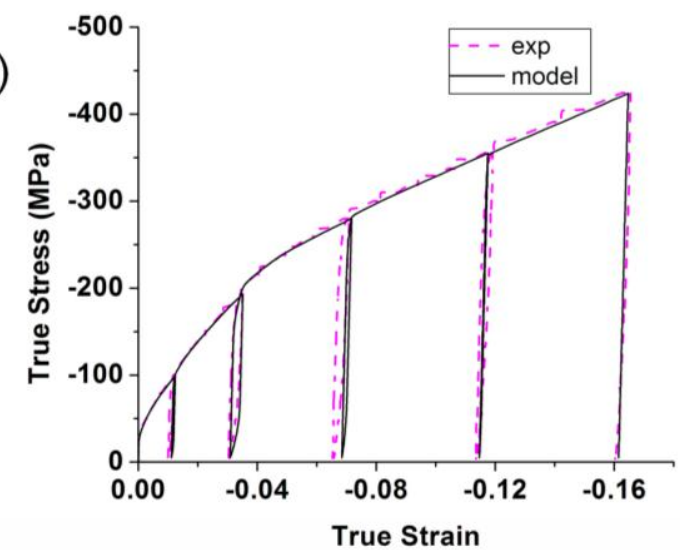

c)

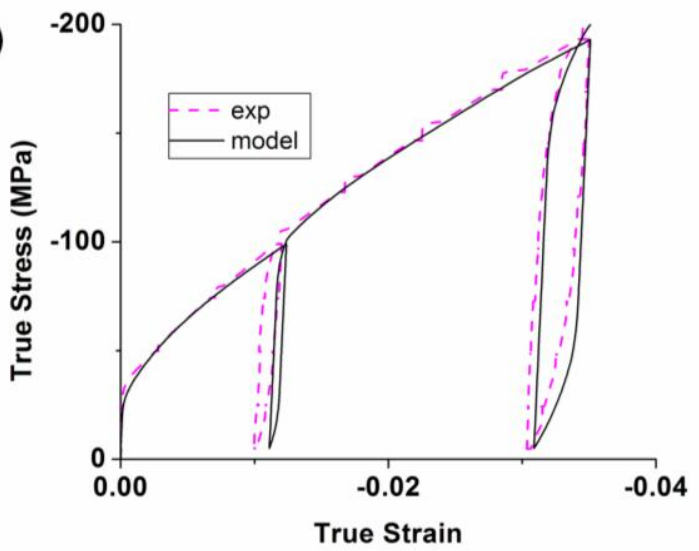

b)

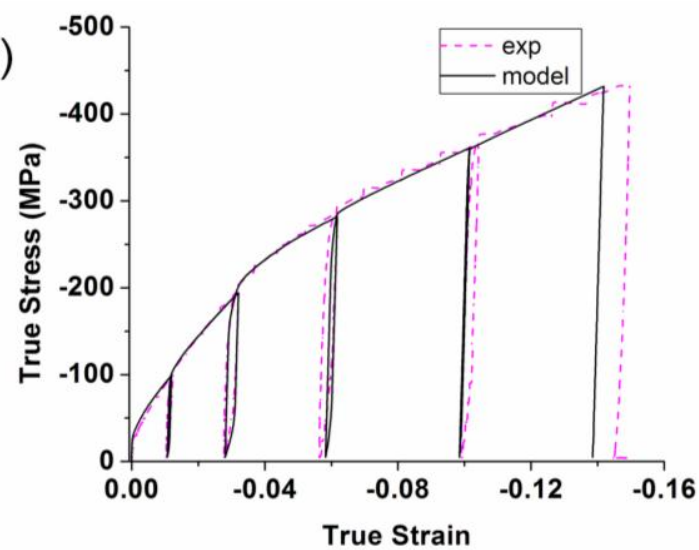

d)

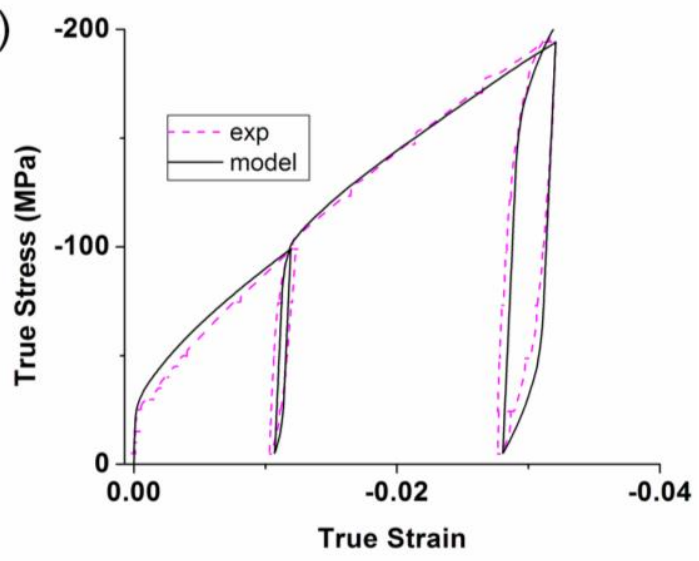

Figure 5.8: EPSC fits superimposed on the experimental stress strain curves for a value of $\beta_{\text {eff }}=0.82$ : a) at room temperature, b) $150{ }^{\circ} \mathrm{C}$. Part of the stress strain curve prior to the transition point has been magnified for clarity c) at room temperature and d) at $150{ }^{\circ} \mathrm{C}$.

A comparison between the simulated and experimental internal strains is shown in Figure 5.5 and 5.6. It can be seen that the model predicts the development of the internal elastic strains reasonably well. The model predicts the (210) oriented grains to be harder and the (111) grains to be softer. Additionally, the agreement between the magnitude of the internal strains predicted by the model and that observed experimentally is good. The onset of the Bauschinger effect can be clearly seen as a change in rate of decrease in the 
internal elastic (compressive) strains in the two sets of grains on unloading. Initially, internal strains in both grain sets start decreasing at the same rate. Eventually, the soft (111) grains diminish their share of internal elastic strain, in the reverse direction, indicating that they are undergoing plastic deformation in tension. On comparing successive unloads, it can be seen that this transition in strain accommodating behavior after unload occurs at higher stress levels when the unloads are prior to the plastic transition $(-250 \mathrm{MPa})$ indicating that the magnitude of the Bauschinger effect is increasing. On unloads performed beyond the plastic transition, the transition in the slope of the internal elastic strains, during unloading, starts at lower stresses and, thereby, indicating a decrease in the Bauschinger effect. Of course this decrease in the Bauschinger effect is over predicted in the internal strains of the model, as this has not been well captured even in the predicted stress-strain curves.

\subsection{Discussion}

CoZr has been studied in detail by several researchers. However, most of the studies are concerned with either the anomalous ductility of this alloy $[16,36]$ or the observed yield strength anomaly $[37,38]$. The Bauschinger effect in this alloy has not been studied previously.

\subsubsection{Bauschinger effect}

As seen from Figures 5.3 and 5.8, the EPSC model predicts the Bauschinger effect prior to the plastic transition (secondary transition) reasonably well. Since materials deforming with an insufficient number of independent slip systems develop large intergranular stresses, these play a dominant role in the observed Bauschinger effect. Past 
the transition point, the intergranular stresses are reduced, since the plastic deformation is more homogeneous. This causes the Bauschinger effect to decrease, an effect that is also captured by the model. It should be noted that the value of $\beta_{\text {eff }}$ (Eq. 4) needed to be adjusted to provide a more compliant interaction in order to describe the experimental behavior.

To further validate this theory, we modeled the monotonic stress strain curve using only the $<111>\{110\}$ slip mode, typical of disordered BCC metals. Although inappropriate for B2 compounds like CoZr [16], this case is informative, since the model predicts no Bauschinger effect during compression-unloading cycles. Rather, it predicts linear elastic unloading, all the way to zero stress. This shows that, when the material has sufficient (i.e. 5) independent slip systems, the deformation is so homogeneous that the intergranular stresses are not large enough to trigger reverse yielding prior to complete unloading, i.e. it would require reverse loading. This result confirms that, when sufficient deformation modes are present, the Bauschinger effect is mainly be due to intragranular (dislocation-based) stresses, and the magnitude in single and polycrystals is expected to be the same, as observed by Paterson [39] in $\mathrm{Cu}$.

In the present case, the EPSC model predicts, for both experimental conditions, the Bauschinger effect prior to the transition point very well but under predicts the Bauschinger effect past the transition point (Figs.5.3 and 5.8). This shows that a model accounting for intergranular back-stress can capture the Bauschinger effect pre-transition, but dislocation based (intragranular) back-stresses will be need to be incorporated to capture the post-transition behavior. By the time the secondary slip mode is activated, a well-developed substructure is expected due to the multiple slip activity predicted by the 
current model results and due to large strains [15]. It is proposed that long-range, albeit intragranular, internal stresses due to such a dislocation substructure will be increasingly important at higher strain levels. Combining a grain-level constitutive model, which accounts for these dislocation-based back stresses, with the present EPSC model, which now accurately accounts for the role of intergranular stresses, would be expected to capture the behavior at all strain levels (before and after the transition).

\subsubsection{Yield Strength anomaly}

Previous studies of CoZr report a room temperature yield point in the range of -55 to $150 \mathrm{MPa}[16,36,37]$. It is also reported that a yield strength anomaly exists in this temperature range, i.e. the yield strength increases with temperature $[36,37,40]$. Yield strength anomalies have been reported in B2 compounds in the past. In cases where <111> slip has been found to occur, the anomalies have been attributed to the dissociation of $<111>$ dislocations into super-partials resulting in cross slip locking on a plane on which the APB energy is lower [41].Other models proposed include the vacancy hardening model for FeAl [42] and dynamic strain ageing (DSA) for CoTi [43]. A core dissociation model has been proposed for Co-based B2 intermetallics like CoZr, CoTi and CoHf [44].

The room temperature yield point obtained in the present study is -50 MPa which is agreement with results from the literature. However, the yield strength decreases to -45 $\mathrm{MPa}$ at $150{ }^{\circ} \mathrm{C}$. The present results differ from previous results in the fact that an anomaly is not exhibited in the yield strength. Rather the material strain hardens more rapidly at the higher temperature after yielding. The current and previous results can be reconciled as follows. 
Even if the secondary deformation shown to be active in $\mathrm{CoZr}$ is considered to be $<111>$ slip, the anomalous strain hardening is observed prior to the activation of the secondary mechanism and hence cross-slip locking on a plane with a lower APB energy can be ruled out. Also, CoZr stress-strain curves do not show any serrations or load drops which are considered signatures of DSA and have been observed in similar compound CoTi.

The core dissociation model seems to be most appropriate to explain the anomaly observed in strain hardening. According to this model, the core of the $<001\rangle$ screw dislocation spreading on the glissile $\{110\}$ plane transforms into the core of the $<001>$ screw dislocation spreading on the sessile $\{001\}$ plane with the help of stress and temperature [44]. This results in anomalous strengthening. The fact that $\mathrm{CoZr}$ is elastically isotropic makes the lattice shear resistance along the $\{001\}<001>$ extremely low facilitating the core spread on the $\{001\}$ plane [44]. The reason that anomalous strengthening occurs past the yield in the present studied may be attributed to the fact that the yield point in this study is very low. It is known that the core dissociation mechanism depends on the temperature and applied stress. It is possible that the stress needed to activate this mechanism is reached only past the yield point which explains why the yield point $150^{\circ} \mathrm{C}$ is lower than the room temperature yield point.

\subsection{Conclusions}

When only the primary slip mode (which does not provide 5 independent slip systems) in CoZr is active, the buildup of intergranular stresses is high and is primarily responsible for the observed Bauschinger effect. It can be speculated that a similar result 
will be obtained in other alloys with an insufficient number of independent slip systems. Because the material is strongly plastically anisotropic, when deforming only by cube slip, the EPSC model (without the incorporation of any dislocation-based back-stresses) reasonably predicts the macroscopic Bauschinger effects, by considering only the intergranular stresses. The magnitude of the Bauschinger effect increases as the interaction stiffness increases, i.e., tends to an iso-strain condition.

Beyond stresses high enough to activate the secondary slip system, the stress distribution among the grains is more uniform and, hence, the Bauschinger effect due to intergranular stresses gradually disappears, as predicted by the model. However, the Bauschinger effect observed experimentally does not diminish so rapidly. This suggests that intragranular back-stresses due to dislocations must be taken into account and such a modeling effort is presently underway.

The room temperature and mildly elevated temperature tests yield a similar level of Bauschinger effect. Contrary to prior studies, a yield strength anomaly has not been observed; rather, an anomaly has been observed in the hardening rate and the plastic (primary to secondary slip mode) transition. The core dissociation model seems appropriate to explain the characteristics of the observed anomaly.

No evidence of a phase transformation (within the detectability limit of the technique) was observed supporting the argument that the ductility observed in CoZr is due to the activation of a secondary slip mode. 


\subsection{Acknowledgements}

This work was supported by the National Science Foundation through a CAREER Grant

DMR-0547981. This work has benefited from the use of the Lujan Neutron Scattering Center at LANSCE, which is funded by the Office of Basic Energy Sciences (DOE). Los Alamos National Laboratory is operated by Los Alamos National Security LLC under DOE Contract DE-AC52-06NA25396. The authors would like to thank Mr. James Wollmershauser for helpful discussions and Mr. Cecil A. Carmichael, Jr. and Dr. Evan Ohriner (Oak Ridge National Laboratory) for performing the arc-casting and hot extrusion of the CoZr alloy, respectively.

\subsection{References}

1. J. Bauschinger: Mitt.Mech.-tech Laboratorium K. Technischen Hochschule Munchen, 1886, 13, 5, p. 31.

2. E. Heyn: Metall und Erz, 1918, 22, pp. 441-2 and 436-41.

3. N. Thompson and N. J. Wadsworth: Advances in Physics, 1958, 7, p. 72.

4. N.H. Polakowski and E.J. Ripling : Strength and Structure of Engineering materials, Prentice and Hall, Englewood Cliffs, NJ, 1966, pp.144-146.

5. H.J. Gough, S.J. Wrights and D. Hanson: Aeronautical Res. Ctttee,, 1924, p. R. and M. No.995.

6. G. Sachs and H. Shoji: Z fur Physik, 1927, 45, p.775.

7. C. Zener: Elasticity and Anelasticity of Metals, The University of Chicago Press, Chicago, IL, 1948, pp 145-146. 
8. E. Orowan: Causes and Effects of Internal Stresses in Internal Stresses and Fatigue in Metals symposium, 1958, pp.59-80.

9. T.M. Wu: Investigation of the Bauschinger effect in metals, MIT Master's Thesis, 1958.

10. J. R. Canal: Investigation of the Bauschinger effect in Copper, MIT Master's Thesis, 1960.

11. G. I. Deak: A study on the causes of the Bauschinger effect, MIT Doctoral Thesis, 1962.

12. A. Seeger and M. Wilkens, in Realstruktur und Eigenschaften on Reinststoffen (edited by E. Rexer), Vol. 3, Akademie, Berlin, 1967, p. 31.

13. J. C. Grosskreutz and H. Mughrabi: in Constitutive Equations in Plasticity (edited by A. S. Argon), MIT Press, Cambridge, MA, 1975, pp. 251.

14. H. Mughrabi, F. Ackermann and K. Herz: in Fatigue Mechanisms, ASTM STP 615 (edited by J. T. Fong), ASTM, Philadelphia, PA, 1979, p.69.

15. H. Mughrabi: Acta Metallurgica, 1983, 31(9): pp. 1367-1379.

16. J.A. Wollmershauser, C.J. Neil and S.R. Agnew: Metall. Mater. Trans. A, 2010, 41A(5), pp.1217-1229

17. T.Yamaguchi, Y. Kaneno and T. Takasugi: Scripta Materialia, 2005, 52(1), pp. $39-44$.

18. H.R. Wenk, L. Lutterotti, and S. Vogel: Nuclear Instruments and Methods in Physics Research Section A: Accelerators, Spectrometers, Detectors and Associated Equipment, 2003, 515(3), pp. 575-588. 
19. M.A.M. Bourke, D.C. Dunand and E. Ustundag: Applied Physics a-Materials Science \& Processing, 2002, 74, pp. S1707-S1709.

20. B. Clausen: SMARTSware Manual, The Regents of the University of California, Los Alamos, NM, 2003.

21. R. Von Dreele: Journal of Applied Crystallography, 1997, 30(4),pp. 517-525.

22. P.A. Turner and C.N. Tome: Acta Metallurgica, 1994, 42(12), pp. 4143-4153.

23. J.D. Eshelby: Proceedings of the Royal Society of London Series A Mathematical and Physical Sciences, 1957, 241(1226), pp. 376-396.

24. I. Baker: Materials Science and Engineering A, 1995, 193, pp.1-13.

25. M. Berveiller and A. Zaoui: Journal of the Mechanics and Physics of Solids, 1978, 26(5-6), pp. 325-344.

26. P. Franciosi, M. Berveiller and A. Zaoui: Acta Metallurgica, 1980, 28(3), pp. 273283.

27. R. Hill: Journal of the Mechanics and Physics of Solids, 1965, 13(2), pp. 89-101.

28. R.A. Lebensohn and C.N. Tomé: Acta Metallurgica, 1993, 41(9), pp. 2611-2624.

29. A. Molinari and L.S. Toth: Acta Metallurgica, 1994, 42(7), pp. 2453-2458.

30. C.N. Tome: Modelling and Simulation in Materials Science and Engineering, 1999, 7(5), pp. 723-738.

31. A. Abel: Mater. Forum., 1987, 10( 1), pp. 11-26.

32. D.S. Agosta, et al.: Journal of Alloys and Compounds, 2002, 346(1-2), pp. 1-5.

33. S.R. Agnew, et al.: Scripta Materialia, 2003, 48(8), pp. 1003-1008.

34. D.W. Brown, et al.: Metall. Mater. Trans. A, 2003, 34A (7), pp. 1439-1449. 
35. J.A. Wollmershauser, S. Kabra, and S.R. Agnew: Acta Materialia, 2009, 57(1), pp. 213-223.

36. Y. Kaneno, et al.:Journal of Alloys and Compounds, 2008, 456(1-2), pp. 125-134.

37. M. Nakamura and Y. Sakka: Journal of Materials Science, 1988, 23(11), pp. 4041-4048.

38. A. Francois and P. Veyssiere: Intermetallics, 1994, 2(1), pp. 9-22.

39. M.S. Paterson: Acta Metallurgica, 1955, 3(5), pp. 491-500.

40. M. Yoshida and T. Takasugi: Philosophical Magazine A, 1993, 68(2), pp. 401417.

41. V. Paidar, D.P. Pope and V. Vitek: Acta Metallurgica, 1984, 32(3), pp. 435-448.

42. E.P. George and I. Baker: Philosophical Magazine A, 1998, 77(3),pp. 737 - 750.

43. M. Wittmann and I. Baker: Materials Science and Engineering A, 2002, 329-331: pp. 206-212.

44. T. Takasugi, et al.: Philosophical Magazine A, 1995, 71(2), pp. 347 - 358. 


\section{Conclusions}

The present work has examined the deformation and fracture behavior of several B2 intermetallic compounds with the main objectives of elucidating the mechanism(s) responsible for measurable ductility in select B2 intermetallics, examining the breadth of this ductility with respect to the B2 family, and understanding the nature of the Bauschinger effect observed in such anomalously ductile B2 intermetallics. In this section, several connections are drawn from the results obtained in the different chapters. For example, while it is shown in chapter 2 that high purity materials with a complete absence of second phases are not responsible for the anomalous ductility, the presence of second phases can be beneficial for the ductility of brittle materials $[1,2]$. However, as shown in chapters 2, 3 and 5, neither of the anomalously ductile alloys, CoTi and CoZr, contain any second phases. Hence, this ductility is also not contingent on the presence of second phases.

\subsection{Reason for anomalous ductility}

Several possible reasons for the anomalous ductility including presence of preferred orientation, departure from order, presence of second phases, phase transformations, twinning, slip of dislocations with <110> and /or <111> Burgers vectors, etc. have been explored.

1. Measurable amounts of second phase impurities are observed in all the ductile B2 rare-earth containing MR compounds. This is not surprising, since many of these alloys are line compounds. However, it is important to note that the anomalous ductility does not require a high level of phase purity as previously suggested. 
Additionally, the ductility is not due to the presence of second phases, since the amount of ductility was not found to be sensitive to the quantity of second phases within the limit examined. In fact, refractory containing MR compounds CoTi and CoZr were essentially single phase but still exhibited significant ductility.

2. Since none of the $\mathrm{B} 2$ alloys examined have a prominent texture, the possibility that texture is primarily responsible for the anomalous ductility is eliminated. However, $\mathrm{AgY}$ was found to have a moderate texture and this may be the reason why it is the most ductile MR compound observed till date. In fact, polycrystal plasticity modeling confirms that the observed texture will lower the flow stress below that required in a randomly textured aggregate.

3. It has been experimentally verified using XRD that the MR compounds are fully ordered. In fact, the order parameters of the most ductile alloys (AgY and CoZr) were determined to be 1 . Thus, unlike some other intermetallic compounds, they do not require disorder or departure from stoichiometry to exhibit ductility.

4. Despite the fact that several different post deformation and in-situ diffraction techniques (TEM, EBSD and neutron diffraction) were utilized (see results in Chapters $3 \& 5$ ), , no evidence was found for phase transformations or twinning in any of the anomalously ductile compounds examined. Therefore, the focus of the study was squarely placed on seeking a dislocation-based explanation.

5. The techniques of TEM-based $\mathbf{g} \cdot \mathbf{b}$, EBSD-based IGMA and neutron diffraction combined with EPSC all together verify that the primary slip modes in CoTi are $<100>\{011\}$ and $\langle 100>\{001\}$. However, beyond a transition in strain hardening observed in CoTi and CoZr, secondary slip modes are activated (see Chapters 3 \& 
5 for details). It is hypothesized that this explanation will also hold for other ductile MR compounds (e.g., AgY, CuY and CuDy [3]).

6. Both $\langle 110\rangle$ and $\langle 111\rangle$ dislocations are found to be present in CoTi. A limited amount of $\langle 110\rangle$ or $<111\rangle$ dislocation motion may reduce stress concentrations and delay fracture. The presence of the $<111>\{110\}$ slip mode provides five independent slip systems on its own. The $<110>\{110\}$ and $\langle 100>\{110\}$ (or $<100>\{010\})$ slip modes together provide five independent slip systems.

7. Dislocation self-energy calculations accounting for elastic anisotropy (section 3.5.3) suggest that the choice of slip direction in these alloys is mobility, rather than source, limited.

8. The confirmed presence of hard slip modes (and the lack of other strain accommodation mechanisms) provides a satisfying explanation for the ductility observed in CoTi and extends to other anomalously ductile B2 compounds, like CoZr.

\subsection{Breadth of ductility}

MgR intermetallics were produced and tested to examine the breadth of ductility of the MR family.

1. $\mathrm{MgR}$ intermetallics namely, $\mathrm{MgCe}, \mathrm{MgNd}$, and $\mathrm{MgY}$ are brittle and exhibit a cleavage-type fracture and, thus, are not a part of the anomalously ductile MR family as previously suggested.

2. Even though the anomalous $\mathrm{AgY}, \mathrm{CoTi}$, and $\mathrm{CoZr}$ can exhibit significant tensile ductility, the fracture surfaces of these alloys do not show any signs of gross 
ductility, as observed in $\beta$-brass $(\mathrm{CuZn})$. Rather, the fracture surface indicates a mixed fracture mode (part transgranular cleavage and part intergranular).

3. Two EBSD-based methods are used to determine the crystallography of cleavage facets in the MgR compounds. Both the methods give similar results which supports their equivalence. $\mathrm{MgY}$ and $\mathrm{MgNd}$ are found to cleave near the $\{100\}$ planes and $\mathrm{MgCe}$ near the $\{111\}$ planes.

4. On comparing the cleavage plane observed in MgR compounds with prototypical B2 intermetallics, it is proposed that extrinsic factors, like testing environment, may be affecting the selection of the cleavage plane.

\subsection{Bauschinger effect in anomalously ductile B2 intermetallics}

A large Bauschinger effect is observed in several of the anomalously ductile B2 compounds, such that the material begins to yield in the reverse direction on unloading.

1. When only the primary slip mode is active in CoZr, the buildup of intergranular stresses is large and is chiefly responsible for the observed Bauschinger effect. The reason that large intergranular stresses build up is that the primary slip mode does not provide 5 independent slip systems required to enforce compatibility between neighboring crystals. The initial strain hardening is very steep which indicates that the overall internal elastic strains are building up at a fast rate. It is possible that a similar behavior will be observed in other alloys with an insufficient number of independent slip systems. 
2. Due to the strong plastic anisotropy when only cube slip is active, the EPSC model (without the incorporation of any dislocation-based back-stresses) reasonably predicts the macroscopic Bauschinger effects in $\mathrm{CoZr}$, i.e. by accounting only for the intergranular stresses. The magnitude of the Bauschinger effect increases as the interaction stiffness increases, i.e., tends to an iso-strain condition. A reduced interaction stiffness, below that traditionally used in EPSC, was required to cause the simulated internal stresses to quantitatively agree with the values measured experimentally.

3. At stresses and strains where the secondary slip modes are active, the stress distribution among the grains is more uniform and hence the Bauschinger effect due to intergranular stresses reduces and this is predicted by the EPSC model and seen in the experimental internal elastic strains. However, while the magnitude of the experimental Bauschinger effect reduces, it does not decrease to the extent predicted by the model, indicating that intragranular back-stresses due to dislocations are becoming important and need to be incorporated in the model in order to fully capture the observed effects of strain path change in these materials.

4. Finally, it is noted that the polycrystal plasticity modeling employed in this work has implications for fracture. If the fracture mechanism is activated when a critical stress level is reached within individual grains, the model may be directly employed. If a more detailed intragranular stress variation is required, e.g. due to dislocation pile-ups, the methods described in the future work (section 7.3) could be relevant. Finally, if immediate grain neighborhood 
effects are required, e.g. to interact with an intergranular fracture criterion, one of the so-called n-site [4] or full-field crystal plasticity models [5, 6] would be required to make advances.

\subsection{References}

1. Sauthoff G. Plastic Deformation, in: Westbrook JH, Fleischer RL (Eds.). Intermetallic Compounds: Principles and Practice. Vol.1. New York: John Wiley \& Sons, Ltd.; 1995. p. 911-934 [chapter 39].

2. Evans AG, and Langdon TG. Prog. Mater. Sci. 1976.; 21:171.

3. Gschneiner K, et al. Nature Mater. 2003; 2:587-590

4. Mu S, et al. Advanced Engineering Materials 2010; 12(10): 1008-1014.

5. Kanjarla AK, Houtte PV and Delannay L. International Journal of Plasticity. 2010; 26(8): 1220-1233.

6. Lebensohn RA, et al. Acta Materialia 2008; 56(15): 3914-3926. 


\section{Future Work}

The present study has raised new questions regarding the deformation behavior in B2 intermetallic compounds.

\subsection{A brief discussion of the implications of the present conclusions on the understanding of yield anomaly in B2 compounds}

Most materials become softer as the temperature is increased. However, many intermetallic compounds are known to exhibit a yield anomaly, i.e., there yield strength increases with temperature. This phenomenon can be very useful in high temperature structural applications. The yield anomaly in $\mathrm{L1}_{2}$ structured $\mathrm{Ni}_{3} \mathrm{Al}$ has been studied by several researchers and is now generally attributed to the Kear-Wilsdorf lock [1-3]. The lower APB energy on the $\{100\}$ plane provides a driving force for thermally activated cross slip of the $\langle 110\rangle$ dislocations from the $\{111\}$ planes to the $\{100\}$ planes [4].

Many B2 intermetallics also exhibit an anomaly either in their yield strength $[5,6]$ or in their strain hardening [7]. No common mechanism is capable of explaining the yield anomaly in the different B2 intermetallics [8]. In cases where $<111>$ slip has been found to occur, like $\mathrm{CuZn}$, explanations similar to the Kear-Wilsdorf lock have been proposed where cross-slip occurs from the $<110>$ to the $<112>$ planes [9]. However, these theories have shortcomings since the driving force for this cross-slip is very low and in some cases, like FeAl, non-existent $[10,11]$. Baker proposed a vacancy hardening model to explain the yield anomaly in FeAl and this model captures the experimental observations well [12, 13]. However, quenching experiments carried out on $\mathrm{CuY}$ [7] and CoTi [14] 
have shown that the room temperature yield strength is independent of the vacancy concentration, and hence, vacancy hardening cannot explain the yield anomaly in these alloys.

A core dissociation model has also been proposed to explain the anomaly in Co-based B2 intermetallics like CoTi, CoZr and CoHf [15]. According to this model, the core of the $<001>$ screw dislocation spreading on the glissile $\{110\}$ plane transforms into the core of the $<001>$ screw dislocation spreading on the sessile $\{100\}$ plane with the help of stress and temperature. This results in anomalous strengthening. Experimental evidence of core-spreading has been provided using HRTEM studies at $773^{\circ} \mathrm{K}$ which is below the yield point peak [16]. On the other hand, Wittman and Baker [14] have proposed that dynamic strain aging (DSA) can explain many of the features of the yield anomaly in CoTi such as negative strain rate sensitivity. However, after examining their data, it can be seen that they were characterizing the transition in strain hardening and not the yield point of CoTi [17]. Recent work on CuY has shown that the yield strength is unaffected by increase in carbon concentration [7]. Hence, DSA has been ruled out as the primary mechanism responsible for the yield strength anomaly in that alloy. A similar experiment on CoTi would prove useful in determining whether DSA plays a role in the yield strength anomaly.

The previous work needs to be reconsidered in light of the recent finding that CoTi and CoZr exhibit both $<111>$ and $<110>$ type slip in addition to $<100>$ slip. However, these secondary mechanisms have been shown to be active only at higher strains and stresses, beyond the transition in strain hardening, while the anomalous strengthening behavior is seen to occur just past the yield point in CoZr (-see Chapter 5) and at the yield 
point in CoTi [18]. While part of the anomaly beyond the transition may be ascribed to $<111>$ and or $<110>$ dislocations (due to thermally activated core-spreading, cross-slip locking, etc.), a comprehensive model must also account for the strengthening of the $<100>$ slip modes with temperature.

The core dissociation model seems to be appropriate to explain the anomaly observed in CoTi. However, specifics of this model remain obscure. $\mathrm{C}_{44}$ decreases with increase in temperature [19] and this facilitates core spreading on the $\{100\}$ planes. Since the slip plane is usually of the $\{110\}$ type, this non-coplanar core-spreading is expected to cause the yield stress to increase. The core-spreading may be reversible, since with decrease in temperature the value of $\mathrm{C}_{44}$ increases. However, Wittman and Baker [14] have shown that it can be irreversible. If the driving force for core spreading is related to a lower energy of the dislocation core on specific planes and the core spreading is thermally activated, then the anomaly would be expected to be irreversible. A detailed examination of the core structure of all of the active deformation modes as a function of temperature and stress is needed to gain an insight into the specific mechanism(s) responsible for the yield anomaly in these intermetallics.

\subsection{Dislocation core structures and mobilities}

The nature of the dislocation core structure in anomalously ductile B2 alloys, particularly the $\langle 110\rangle$ and $\langle 111\rangle$ dislocations, is unclear. It is possible that the core structure of these dislocations may elucidate the reason for their early activation in select B2 alloys. High resolution transmission electron microscopy (HRTEM) studies can be used to analyze the core structure [20-22]. Up to the present, the core structures reported in B2 alloys are without the application of external stresses. It is possible that the 
dislocation core structure changes under the influence of external stresses and this may play a role in determining the mobility of these dislocations and their role in plastic deformation. These aspects can be analyzed using atomistic modeling studies.

\subsection{Incorporation of intragranular stresses in the EPSC model}

It has been said that the litmus test for any complete hardening model/ theory is that it should be able to quantitatively account for the Bauschinger effect [23]. The present study has shown that without the incorporation of intragranular stresses the EPSC model is unable to capture the Bauschinger effect and hence this addition will improve the model significantly. In fact, some progress has already been made in this area in Prof. Agnew's group where a student has presented a version of such a model [24].

\subsection{References}

1. Thornton PH. Metallurgical and materials transactions A 1970;1(1):207.

2. Lall C. Metallurgical transactions A 1979; 10(9):1323-1332.

3. Paidar V, Pope DP, and Vitek V. Acta Metallurgica 1984; 32(3): 435-448.

4. Kear BH and Wilsdorf HGF. Trans. Met. Soc. AIME 1962; 224:382-386.

5. Saka H. Philosophical magazine. A 1985; 51(3): 365.

6. Takasugi T, Izumi O, and Yoshida M. Journal of Materials Science 1991; 26(11): 2941-2948.

7. Becker AT. The yield strength and flow stress anomaly in B2 YCu 2010, PhD. Thesis, Ames, Iowa: Iowa State University.

8. Veyssiere P. Materials science \& engineering. A 2001; 309-310:44-48

9. Umakoshi Y. Acta metallurgica 1976; 24(1): 89.

10. Yoo MH. Acta metallurgica 1988; 36(11): 2935. 
11. Fu CL and Yoo MH. Acta Metallurgica et Materialia 1992; 40(4): 703-711.

12. Baker I. Acta metallurgica et materialia 1995; 43(4): 1723.

13. George EP and Baker I. Philosophical Magazine A 1998; 77(3):737-750.

14. Wittmann M and Baker I. Materials Science and Engineering A 2002; 329-331: 206-212.

15. Takasugi T., et al. Philosophical Magazine A 1995; 71(2): 347 - 358.

16. Shindo D., et al. Intermetallics 1995; 3(2):167-171.

17. Wollmershauser JA, Neil CJ and Agnew SR. Metallurgical and Materials Transactions A 2010; 41A(5):1217-1229.

18. Takasugi T, Yoshida M, and Kawabata T. Philosophical Magazine A 1992; 65(1): $29-40$.

19. Yasuda H, Takasugi T, Koiwa M. Materials Transactions JIM 1991; 32(1): 48-51.

20. Louchet F., et al. Philosophical Magazine A 1988; 57(2): 327-335.

21. Mills MJ and Miracle, DB. Acta Metallurgica et Materialia 1993; 41(1): 85-95.

22. Balk TJ. Philosophical magazine. A. 2001;81(6): 1507-1531.

23. Suresh S. Fatigue of Materials 1998, Cambridge: Cambridge University Press; 98.

24. Wollmershause JA, Clausen B, and Agnew SR. International Journal of Fatigue 2012; 36(1): 181-193. 